\title{
BOUNDS FOR MAPS OF AN INTERVAL WITH ONE CRITICAL POINT OF INFLECTION TYPE II
}

Genadi LEVIN and Sebastian VAN STRIEN
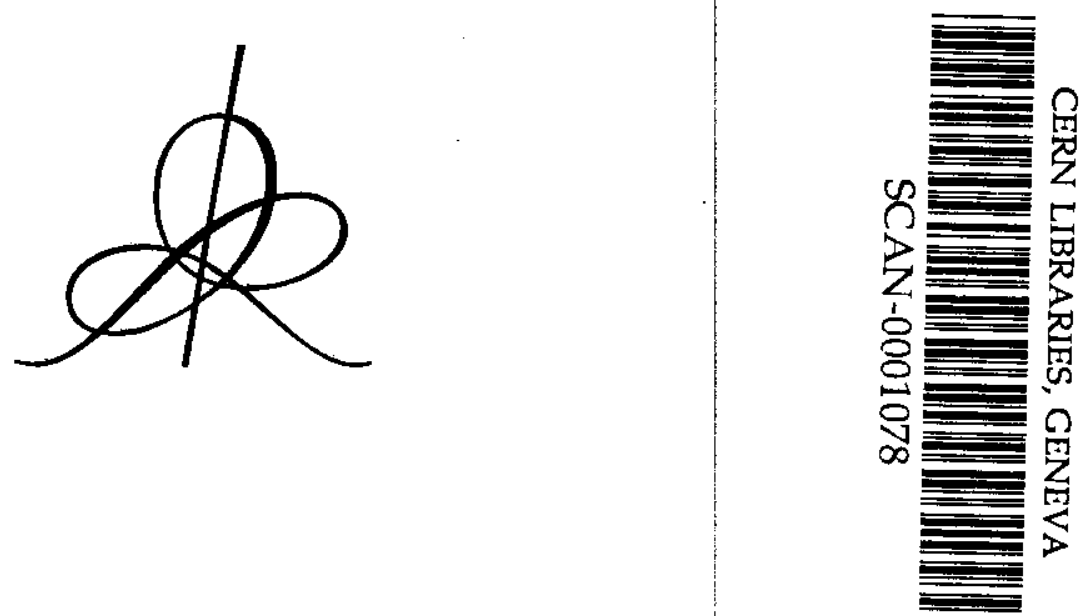

Institut des Hautes Études Scientifiques

35 , route de Chartres

91440 - Bures-sur-Yvette (France)

Octobre 1999

IHES/M/99/82 


\title{
Bounds for maps of an interval with one critical point of inflection type. II.
}

\author{
Genadi Levin, *Hebrew University, Israel ${ }^{\dagger}$ \\ Sebastian van Strien, University of Warwick, England ${ }^{\ddagger}$
}

In this paper we prove complex bounds, duasisymmetric rigidity, absence of invariant linefields and density of hyperbolicity for real analytic maps with one reflecting critical point which are orimtation preserving on mach hranch. For real bounds, see the first part of this paper, [Lo].

\section{Introduction}

Recently anite a few papers appeatred proving complex bomols, local connectivity of Julia sets, absence of invariant linefields and gnasisymmetric rigidity for real polynomial maps.

Let us first discuss the mimodal ase. In 1990 Matens | Mal proved that there are real bounds for a certain seguence of first retum maps to suitable central intervals. In 1990 Sullivan showed that if the unimodal maps are real analytic, infinitely renormalizable and have bounded combinatorics, then their lirst retum majes extend to the complex plane as polynomial-like maps, see [Su] and also [MS]. In 1994 the present authors proved that all real analytic mimodal maps allow such polynomial-like complex extensions for the 'nom-central' first retum maps. 'The proof even gave explicit numerical lower bomnds for the moduli of certain anmuli, [LS1]. For the quarlatic case, different proofs were later provided by [CS1], [LY]. For the smooth case see [Ko]. In 1996 Sands, see [Sia]. improved onr mumerieal bomeds for maps of the form $g\left(x^{t}\right)$ so that the Sohwarzian derivalive of $g$ is negation. This polynomial-like structure is one of the main ingredients in many recent result on smootl muinorlal maps. For example, it is heavily used in the proof that topologically conjugate quadratic maps are in fact (quasisynmetrieally conjugate, see [GS2], also [Ly] and more recently [Sh]. Note that this is the main step in the recent proots that hyperbolic maps are dense in the family $i^{2}+c, c \in \mathbb{R}$. Moreover, complex lomuds learl to the proot of the lowal connectivity or total disconnertedness of Julia sets of some classes of real polynomials [LS1]. [LS2]

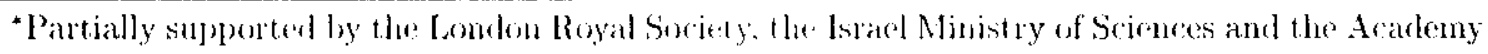
of Sciences and the Arts

tr-mail: levingenath.hujiate.il

fo-mail: strien' 
and ane atso needed in resulls on renormalization and on smenthoness of conjoga ies. sor [Su], [MS], [McM2], and [MP], and essentially simplifies the prowef of absence of

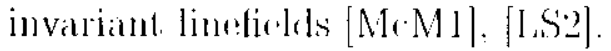

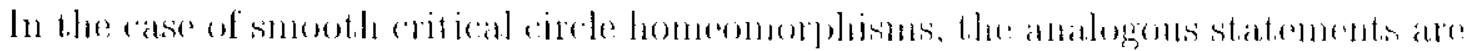

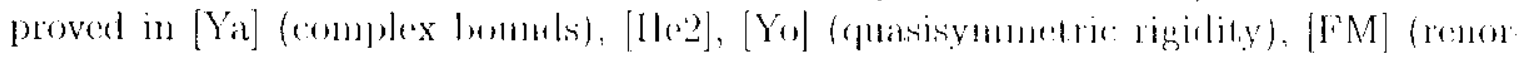
malization).

In this paper we deal with the next natulat alass, which contains smooth covering

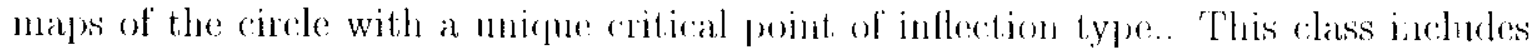
(generalized Amol'd) males of the lorm $f(x)=k \cdot x+a+b \sin (2 \pi x) \bmod 1$ where

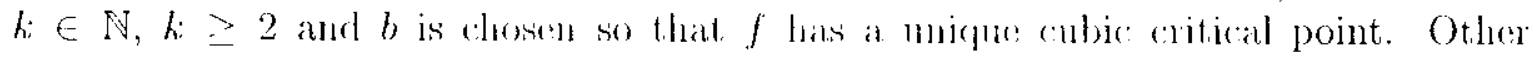
examples are certain real polynomial malss with one non-escaping critical point of inflection type, and also certin Blaschlse prolucts, see the examples below. Just ats critical circle homeomorphisms are in the boundary of the set of diffeomorphisn of the circle, the maps we consider in this paper are in the boundary of the clars of smooth covering maps without critical prints. These covering maps without attracting or neutral periodic orbits and without ritical points are hyperbolically expanding (by a theorem of Mañé), and are quasisymmonrically conjugate to each other. In this paper we will study the metric theory for majs with points of inflection. It turns out that the methods of proof differ substantially from those used in the critical circle case and also quite a bit from the mimodal cast.

It turns out that to prove real and complex bounds for maps with a critical point of inflection type is more involved than for milps with a folding critical point. This is because one no longer has a clynamically relevant symmetry mear the eritical point. (Note that these covering maps (an no longer be remomalizatle.) Therefore we obtain polynomial-like extensions for a secplence of first return matls at moments which are no longer combinatorially defined. As in the cise of critical cirele maps, onr mape atre in general not guasisymmetrically conjugate to affine maps. In this case we no longer have growth of moduli (as in the quadratic (ase), and atso no liomnded geonetry of the postcritical set (as in the critical circle case). In the minimal case we use instearl hat the dynamics does change if we 'move the map up'. In the non-mininal case, a much more general way of proving quasisymmetric rigirlity is used. On method also caut be used to prove quasisymmetric rigidity and density of hyperbolicity for real malytic: unimodal maps (with a folding critical point which is not mexessarily quirliatide) in the non-minimal case.

Let us be more formal and introduce the class $\mathbf{A C}$ which imeludes andy tir: covering maps of the circle. Lot $I$ be an open interval around the peint $c=0$ and $I_{i}$ a (finite) collection of disjoint open intervals inside the interval $l$. AC: is the vass of mills $g: \cup I_{i} \rightarrow I$, for which the following conditions hold.

1. For every $i$, the map $g: I_{i} \rightarrow I$ extends to an orientation pessuving mal analytic: homeomorphism from the closure of $I_{i}$ to the closure of $I$.

2. For every $i \neq 0$, the map $g: \bar{I}_{i} \rightarrow \bar{I}$ is a diffeomorphism, while $\|_{11}$ แlaip $y: \ddot{I}_{0} \rightarrow \bar{I}$ has a unique critical point (i.e., a zero of $g^{\prime}$ ) at the point $c=0 \in l_{10}$, w old onder $\ell \geq 3$. We call $g: I_{0} \rightarrow I$ the central branch, and $I_{0}$ the central interul of $g$. 


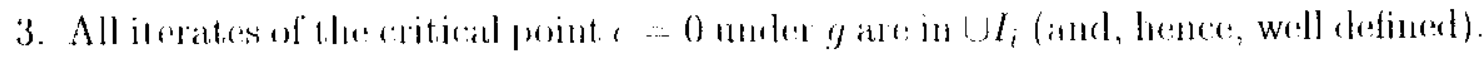

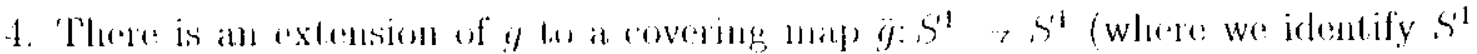

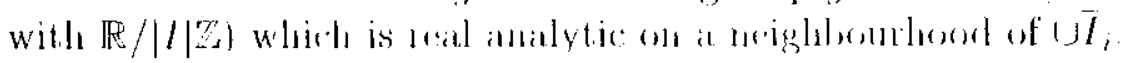

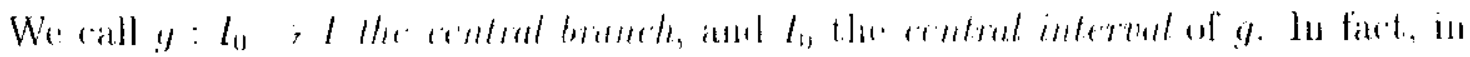

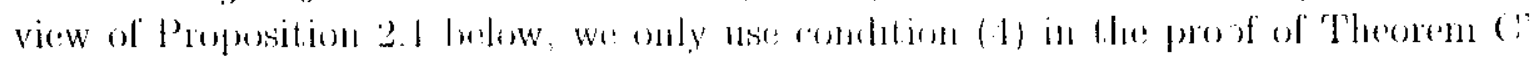

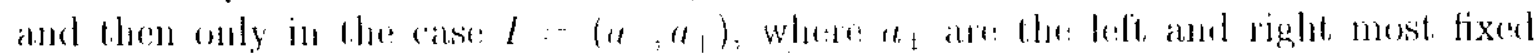
points of $y$.

\section{Examples.}

1. Consirter a pulynomial $P$ with real coefficients. Assume that all except one of its critical point weape to infinity and that the non-escaping critical point $c$ is a real point of inflection type. Moreover, assume that the orbit of $c$ only meets components of $\left\{x \in \mathbb{R} ; P^{\prime}(x) \neq 0\right\}$ on which $P$ is orientation preserving. If we restrict $P$ to a suitable interval of the real axis, it will satisfy the conditions 1-4 (see [LS2] for details).

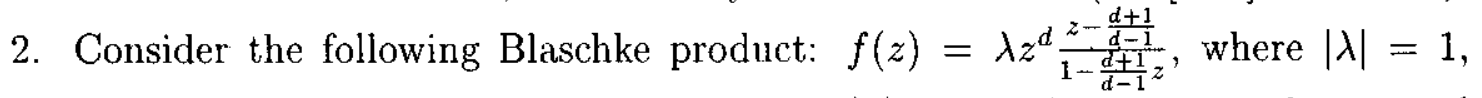
and $d \geq 3$. Then $f$ being restricted to the circle $|z|=1$ and written in the natural coordinate of the circle satisfies the conditions 1-4 above, with $\ell=3$ and with $d-1$ intervals $I_{i}$. Note that $\lambda=f(1)$ is the critical value of $f$ on the circle.

In fact, one can easily generalize our results to more general classes of maps. For example, if $g$ is a piecewise analytic map of the form $g: \cup_{i} I_{i} \rightarrow \cup_{k} I^{k}$ where $\cup_{i} I_{i} \subset$ $\cup_{k} I^{k}$ and $I_{i}$ (respectively $I^{k}$ ) are pairwise disjoint intervals satisfying the analogous properties 1-4 from the class $\mathbf{A C}$, then the branches of first return map to $I_{0}$ containing points of $\omega(c)$ form a map from $\mathbf{A C}$ in the case that $\omega(c)$ is minimal. Otherwise one can again use methods from Sections 10-12 of this paper.

Maps from AC have no wandering intervals and only a finite number of attracting and parabolic orbits, see the next section. In this paper we shall mainly deal with complex bounds for such maps. By this we mean that one has geonctric estimates for the (quasi) polynomial-like maps or box mappings obtained by taking complex extensions of suitable first return maps to small interval neighbourhoods of the critical point. Note that the domain of such return maps consists of infinitely many components. The interesting case is when $c$ is recurrent (i.e.. $c \in \omega(c)$ ) in which case $c$ is contained in one of the components of the domain. The non-romrent case is much easier, see Theorom A' in Section 10. As is usual in this problem one: laus to distinguish the persistently recurrent and the reluctantly recurent rase. Sinro we are in the real case, this corresponds to the distinction betwern $\omega(c)$ being minimal or not. (That $\omega(c)$ is minimal means that cach orbit in this set is lense. In particular, w(c) is a Cantor set in this case.) If $w(c)$ is minimal then there are only finitely many domains of the first return map containing points of whe and in the next theren we restrict our attention to these. The nom-mininal case is discassed below the statement of theorem B.

Theorem A (complex bounds). A muj g $\mathrm{AC}$ such that $\omega(c)$ is minimal has

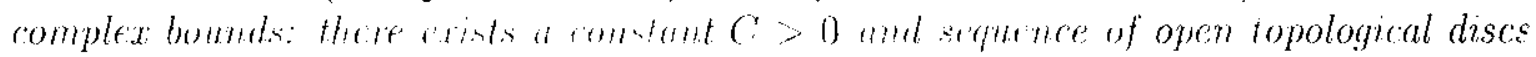

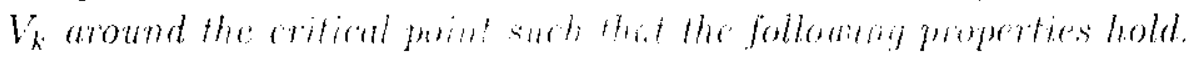




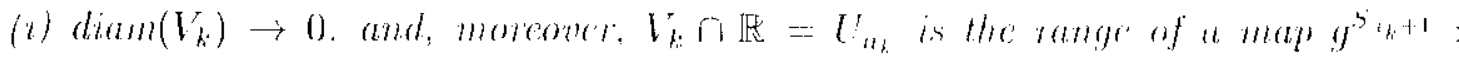

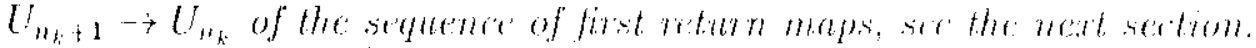

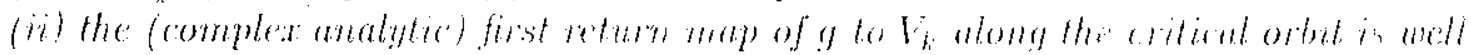

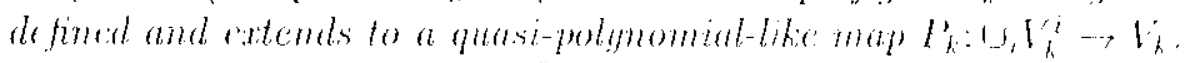

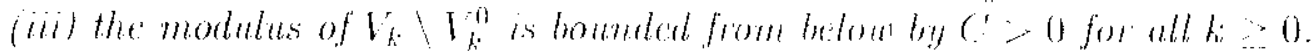

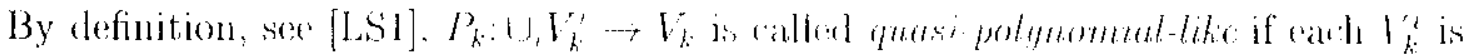

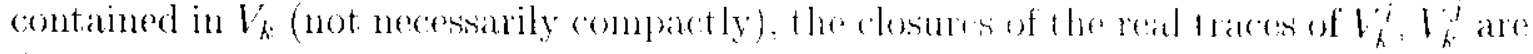
disjoint, if $i \neq j$, and compartly contained in the real tares of $V_{k}$ and $V_{k}: V_{k}^{i} \rightarrow l_{k}$ is a

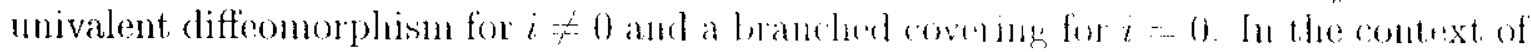

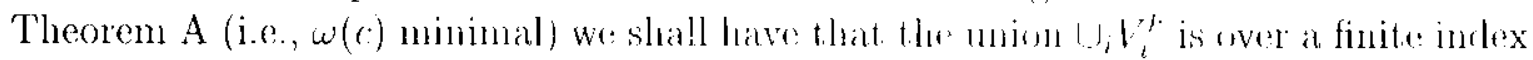
set and that the forward orbit ol calways memains in this moion. Note that the demains

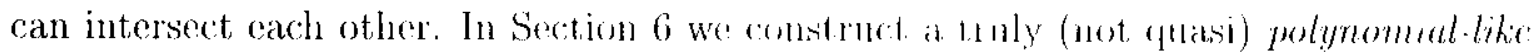
map ([DH], $[\mathrm{LM}]$ ) of arbitratily small dianeter for any $y \in \mathrm{AC}$ with minmal $\omega(\mathrm{e})$, by 'intersecting' the quasi-polynomial-like map with an antificially constructed smooth 'polynomial-like' mape (in a way which is somewhat similar to what was done in [LST1] for mumodal maps). It allows us lo chasiconformally topologically conjugate this polynomial-like map to a polynomial from a family of inercial pelynomials, see Section

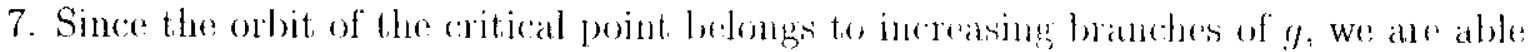
to prove using holomorphic dynamias:

\section{'Theorem B (rigidity)}

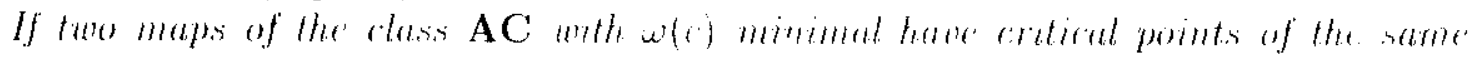

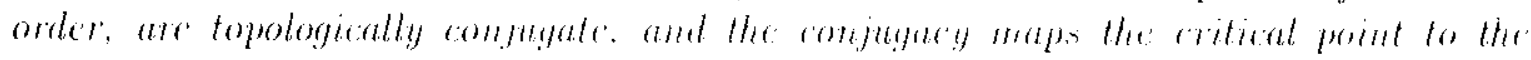

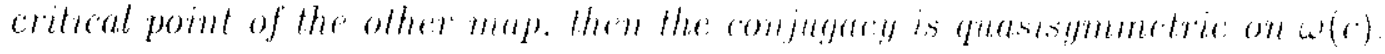

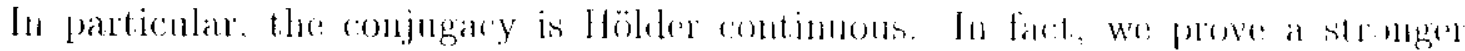

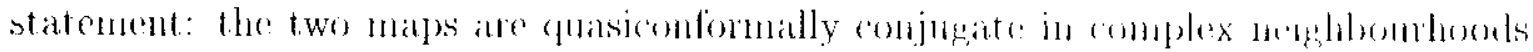

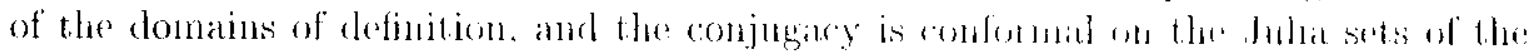
polynomial-like maps mentioned above.

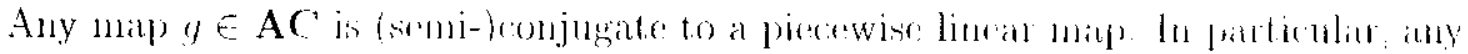

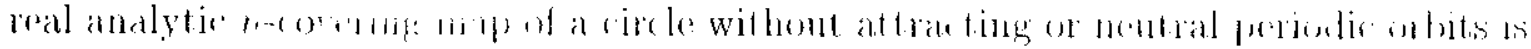

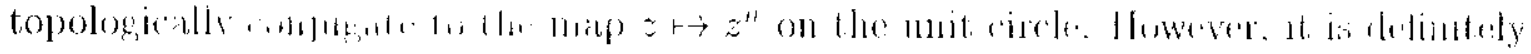

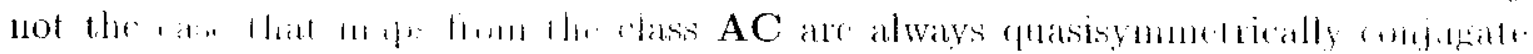

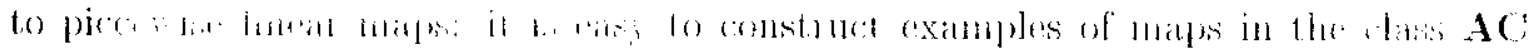

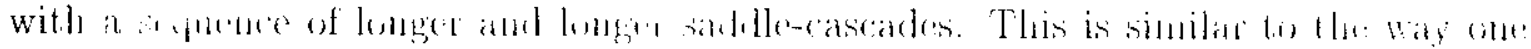

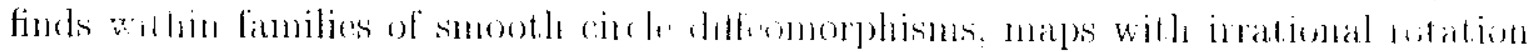

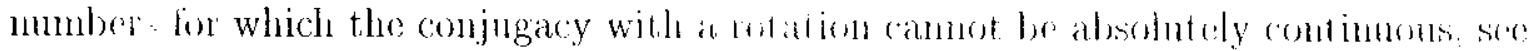
[H(1) o for example [MS].

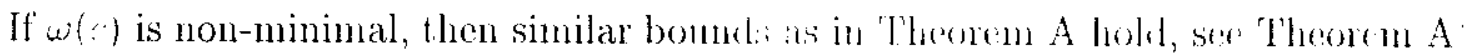
in Section 10. In fact, if $c$ is recurrent and non-minimal then we constinet a kind of

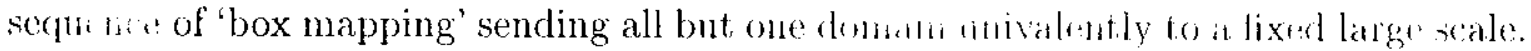

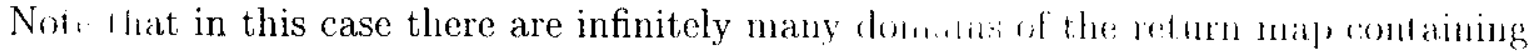
polin of $\omega(c)$, which shall complicate the situation wusterably. 
If $\omega(c)$ is non-minimal then the analogous statement to Theorem $\mathrm{B}$ also holds, see Theorem B' in Section 11. However, in this case we have to assume that $\omega(c)$ contains no parabolic periodic points. Note that this is always the case if $\omega(c)$ is minimal. (As mentioned before, the method in the non-minimal case is very general and also applies to real analytic unimodal maps.)

It is very easy to show that the set of Axiom A maps in the class AC is dense, see Section 12. It is much less obvious that Axiom A maps are dense within any analytic family. However, repeating an argument used by O. Kozlovski in his thesis [Ko], we shall deduce from Theorems A, B and A', B' (see Section 12):

\section{Theorem C (stability)}

Within any non trivial real analytic family of regular maps $g \in \mathbf{A C}$, either each $g$ from this family has a neutral periodic orbit or the Axiom A maps within this family are dense.

Here we say that $g$ is a regular map if each parabolic periodic orbit $O(p)$ of $g$, is either disjoint from $\omega(c)$ or one has $\omega(c) \subset O(p)$. Note that if $\omega(c)$ is minimal, then $g$ is automatically regular. (Actually, in Section we shall prove a slight generalization to Theorem C.) It follows from Theorem C, that within families of maps as in Examples 1 and 2 above, Axiom A maps are dense (the maps as in these examples are regular). Also, $g$ is regular if for example $S g<0$ or if $g$ extends to a (generalized) polynomial-like map with one critical point of odd order.

A consequence of Theorem $\mathrm{A}$ is:

\section{Corollary}

1. The Julia set of polynomial-like maps as above is totally disconnected and carries no invariant line fields.

2. The Julia set of the rational function introduced in Example 2 is locally connected. Proof: Follows as in [McM1], [LS2] from the complex bounds.

In fact, the absence of invariant linefields proved in this corollary is $\varepsilon$ very important ingredient in the proof of Theorem $B$.

Another consequence of the proofs of Theorem B and B' is

Theorem B" (rigidity) Assume that two maps of the class AC have no parabolic or attracting periodic points, and there is a conjugacy which maps the critical point of one of these maps to the critical point of the other map. Then the conjugacy is quasisymmetric. In fact, this conjugacy can be extended to a quasiconformal homeomorphism on the plane which is a conjugacy on a 'necklace neighbourhood' of the dynamical interval; this neighbourhood consists of infinitely many dicjoint topological discs. 
The above results should provide the basis for a study of "attractors", and "universality structure" in the class AC.

Acknowledgements. The authors wish to thank Welington de Melo, Greg Swiatek, and also Alex Eremenko and Misha Sodin for valuable discussions. We are grateful to Greg Swiatek and Feliks Przytycki for listening to detailed expositions on some results of this paper. This IHES preprint is a slightly expanded version of a paper submitted for publication (Theorem B" was added). We also would like to thank the refaree for several valuable remarks, for pointing out one error in a construction used in Theorem $\mathrm{C}$ in the Warwick preprint version of the paper. We are also grateful to the refere for insisting that we give more detail in the proof of Theorem B' and that we make the paper more readable. The research for this paper was initiated while the first author visited the Mathematics Department of Warwick. A substantial part of the paper was written during a stay of the first author at the Mathematics Department of University of Princeton and the Mathematics Department of Penn State University (March-June 1998). The first author sincerely thanks these Institutions for their hospitality. Both authors would like to thank IHES: Theorem B" was added while they visited IHES in the latter part of September 1999.

\section{Some background}

Proposition 2.1 Maps g satisfying conditions 1-3 of the class AC do not have wandering intervals and have only finitely many non-expanding periodic orbits.

Proof: Any such map induces a covering map $\bar{g}: S^{1} \rightarrow S^{1}$. Indeed, first restrict $g$ to the interval $\left[a_{-}, a_{+}\right]$where $a_{ \pm}$are the left and right most fixed points of $g$. Points which are mapped eventually outside this interval will also eventually map outside the domain of definition of $g$. Then simply extend $g$ to a piecewise increasing map defined on $\left[a_{-}, a_{+}\right]$with a unique critical point at $c$. If the (closures of) domains of $g$ are all disjoint then we can make sure that $\bar{g}$ is $C^{\infty}$. If two domains of $g$ intersect, then $\bar{g}$ will be $C^{\infty}$ except at a finite number of preimages of a fixed point (where the left and right derivative of $g$ will differ). In particular, $\bar{g}$ will be in the class $C^{1+Z}$ (its derivative is Zygmund) and so it follows from the comments below Theorem IV.A in [MS] that $\bar{g}$ has no wandering intervals. The map $\bar{g}$ is not in the class $C^{1+z}$ so one cannot apply Theorem IV.B from [MS] immediately. Note however, that it fails this smoothness condition only at a finite number of preimages of a fixed point. The intervals $U_{n}$ from Lemma 10.3 on page 323 of [MS] do not intersect these preimages of the fixed point, and so along the pullback $\left\{U_{0}, \ldots, U_{n}\right\}$ needed in the proof of Proposition 10.1 of [MS] one still has the required cross-ratio estimate. It follows that Theorem IV.B from [MS] still holds. Hence periodic attractors and parabolic orbits of $\bar{g}$ all have period less than some number $N$ (which depends on $g$ ). Since $g$ is analytic on each branch, it follows that $g$ has only finitely many non-expanding periodic orbits.

Let us introduce some notation and background. 
If $I \subset J$ are intervals then $|I|$ denotes the length of $I$. The left and right endpoints of $I$ are denoted by $\partial_{l} I$ and $\partial_{r} I$. The left and right components of $J \backslash I$ are denoted by $l(J \backslash I)$ and $r(J \backslash I)$.

If $J \subset T$ are two intervals and $L, R$ are the components of $T \backslash J$ then we define $C(T, J)$ to be the cross-ratio of this pair of intervals:

$$
C(T, J)=\frac{|J||T|}{|L||R|}
$$

Often we prefer to work with $C^{-1}(T, J)=1 / C(T, J)$. Cross-ratios play a crucial role in all recent metric results in real interval dynamics. If $f \mid T$ is a diffeomorphism and $S f<0$ (where $S f$ is the Schwarzian derivative of $f$ ) then [MS]

$$
C^{-1}(T, J) \geq C^{-1}(f(T), f(J)) .
$$

In our case we shall apply this to maps $f$ of the form $g^{n}$. If $S g<0$ then also $S g^{n}<0$ so the previous inequality applies when we take $f=g^{n}$ provided $g^{n} \mid T$ is a diffeomorphism. We will use the following amusing extension of this classical fact:

Lemma 2.1 Consider a map

$$
F(x)=[h(x)]^{\ell}
$$

where $\ell>1$ is an odd number, and $h$ is a local diffeomorphism neat $x=0$, so that $h(0)=0$, and so that $h$ increases cross-ratios. Then for each $\epsilon>0$ there exists $\Delta>0$ such that the following holds. Let $L$ be the left component of $T \backslash J$, so that if we denote $L=\left(x_{1}, x_{2}\right)$, then

$$
x_{1}<0<x_{2} \text { and }\left|x_{2}\right| \geq(1+\epsilon)\left|x_{1}\right|
$$

and assume moreover that

$$
|T|<\Delta
$$

Then

$$
C^{-1}(T, J) \geq C^{-1}(F(T), F(J))
$$

Proof: See [Le] and use a continuity argument.

If $h$ is only real analytic then a similar statement holds, see Lemma 4.3.

If $I$ is an interval near the critical point $c$, then we shall denote by $I^{g}$ the interval $g(I)$ near the critical value. We shall say that the smaller interval $J$ is well inside $I$, if the length of every component of $I \backslash J$ is at least $C_{*} \cdot|J|$, where $C_{*}>0$ is a universal constant (i.e. does not depend on the integer $n$ introduced below). Often we shall take $C_{*}$ to be equal to something like $C / 10^{\ell}$ where $C$ is the constant from Theorem 2.1. If we can take $C_{*} \geq 2$ then we say that $J$ is deep inside $I$.

Consider now a map $g$ as in the introduction and so that the critical point is recurrent. As usual, define a sequence of first return maps

$$
g^{S_{n}}: U_{n} \rightarrow U_{n-1}
$$


inductively as follows. We set $S_{1}=1, U_{1}=I_{0}$, and $U_{0}=I$. If $U_{n-1}$ is defined, then $g^{S_{n}}: U_{n} \rightarrow U_{n-1}$ is the central branch of the first return map to $U_{n-1}$ (i.e. $U_{n}$ is the component of the domain the first return map to $U_{n-1}$ which contains $c$ ).

Let us say that the map $g^{S_{n}}: U_{n} \rightarrow U_{n-1}$ has a central return if $g^{S_{n}}(c) \in U_{n}$, and a non-central return otherwise. Sometimes we shall simply say that $g^{S_{n}}: U_{n} \rightarrow U_{n-1}$ is central. Note that if some $g^{S_{n}}: U_{n} \rightarrow U_{n-1}$ has a central return, then by pulling back the central domain $U_{n}$ by the map $g^{S_{n}}$ several times, we always obtain a noncentral return map $g^{S_{m}}: U_{m} \rightarrow U_{m-1}$, where $m>n$ and where the maps are the same, i.e., $S_{m}=S_{n}$. (Otherwise $c$ is in the basin of a periodic attractor.) We always have $S_{n+1} \geq S_{n}$. More specifically, $S_{n+1}=S_{n}$ iff $g^{S_{n}}: U_{n} \rightarrow U_{n-1}$ is central, and $S_{n+1}>S_{n}$ otherwise.

Theorem A of this paper gives the complex analogue of the real bounds which were proved in [Le].

Theorem 2.1 Let $g \in \mathrm{AC}$ and assume that $\omega(c)$ is minimal. Also assume that $g$ increases cross-ratios on intervals on which it acts diffeomorphically. Moreover, assume that one can write $g(x)=g(c)+F(x)$ in a neighbourhood of the critical point $c=0$, where $F$ is as in Lemma 2.1.

Then there exist a positive number $C$, which depends on $\ell$ only, ard an integer $N$, so that for every $n \geq N$, the length of every component of $U_{n-1} \backslash U_{n}$ is equal to at least $C \cdot\left|U_{n}\right|$, whenever the previous map $g^{S_{n-1}}: U_{n-1} \rightarrow U_{n-2}$ is non-central.

Let $g^{S_{n}}: U_{n} \rightarrow U_{n-1}$ be non-central. If $S_{n}>S_{n-1}$ (i.e., $g^{S_{n-1}}: U_{n-1} \rightarrow U_{n-2}$ is non-central again), then we set $k(n)=0$, so that $U_{n-1}=U_{n-k(n)-1}$. Otherwise (if $g^{S_{n-1}}: U_{n-1} \rightarrow U_{n-2}$ is central), there exists a first (maximal) interval $U_{n-k(n)}$, $k(n) \geq 1$, in the chain (cascade) of central returns containing $U_{n}$, so that $S_{n}=S_{n-1}=$ $\ldots=S_{n-k(n)}>S_{n-k(n)-1}$ (i.e., $g^{S_{n-k(n)-1}}: U_{n-k(n)-1} \rightarrow U_{n-k(n)-2}$ is non-central). By Theorem 2.1, $U_{n-k(n)}$ is well inside $U_{n-k(n)-1}$ and also $U_{n+1}$ is well inside $U_{n}$. Let us call $\left\{U_{n} \subset \ldots \subset U_{n-k(n)}\right\}$ the maximal chain of central intervals, which contains $U_{n}$ (we do not exclude the case that $k(n)=0$ ).

We shall use the following statement several times.

Proposition 2.2 Let $g$ be as in the previous Theorem. Let $I \subset U_{n}$ be a component of the domain of the first return map to $U_{n}$ (for example, I could be equal to the central domain $U_{n+1}$ ), and assume that the first return map restricted to $I$ is of the form $g^{s}$ for some $s>0$. Then there exists an interval $\tilde{U}$ containing $I$ such that $g^{s-1}: \tilde{U}^{g} \rightarrow U_{n-k(n)-1}$ is a diffeomorphism.

Proof: Denote $S=S_{n}=\ldots=S_{n-k(n)}$. If $s=S$, then $\tilde{U}=U_{n-k}$. So assume that $s>S$. Applying $g^{S}$ to $U_{n}$ for $N=k(n)+1$ times, we see that $g^{S}\left(U_{n}\right)=U_{n-1}$, $g^{S}(I) \subset U_{n-1} \backslash U_{n}, \ldots, g^{N S}\left(U_{n}\right)=U_{n-k(n)-1}, g^{N S}(I) \subset U_{n-k(n)-1} \backslash U_{n-k(n)}$. In particular, $g^{N S}(I)$ lies in a component $K$ of the first return map to $U_{n-k(n)-1}$ other than the central one. Pulling back $K$ to $U_{n}$, we obtain the interval $\tilde{U}$.

We shall also use the following 
Theorem 2.2 (Mañé) Let $g: N \rightarrow N$ be a $C^{2}$ map where $N$ is a circle or an interval. Let $U$ be an open set containing the critical points of $g$, the parabolic periodic points and also the immediate basins of periodic attractors. Then there exists $C>0$ and $\lambda>1$ such that for each $x$ and each integer $k$ for which $x, g(x), \ldots, g^{k-1}(x) \notin U$ one has $|D g(x)| \geq C \lambda^{k}$.

Proof: For a proof of this statement and a simplified proof of the original result of Mañé, see [MS].

We shall also use the following result from complex analysis. Given a bounded real interval $T$ we shall write $D_{*}(T)$ for the disc which is symmetric with respect to the real line and which intersects the real line exactly in $T$. More generally, if $T$ is a bounded real interval and $\alpha \in(0, \pi)$ then consider a disc $D$ which intersects the real line exactly in $T$ such that $D_{+}=D \cap\{\operatorname{Im}(z)>0\}$ has an external angle with the real line of angle $\alpha$. The set $D(T ; \alpha)$ denotes the union of $D_{+}$and its mirror image with respect to $\mathbb{R}$. (Note that $D(T ; \alpha)$ decreases with $\alpha \in(0, \pi)$ increasing.) The reason these sets play an important role, can be explained as follows. Let $\mathbb{C}_{T}=\mathbb{C} \backslash(\mathbb{R} \backslash T)$. The set $\mathbb{C}_{T}$ carries a Poincaré metric, and with respect to this metric the set $D(T ; \alpha)$ consists of all points whose distance to $T$ is at most equal to some constant $k(\alpha)$. From this interpretation and the Schwarz lemma it follows that if $\phi: \mathbb{C}_{T} \rightarrow \mathbb{C}_{T^{*}}$ is a univalent conformal mapping sending $T$ diffeomorphically to $T^{\prime}$, then

$$
\phi(D(T ; \alpha)) \subset D\left(T^{\prime} ; \alpha\right)
$$

As in $[\mathrm{Su}]$, we shall apply this statement in the following way:

Lemma 2.2 (Schwarz) Let $F: \mathbb{C} \rightarrow \mathbb{C}$ be a real polynomial whose critical points are on the real line and which maps the interval $T^{\prime}$ diffeomorphically onto the interval $T$, then there exists a set $D \subset D\left(T^{\prime} ; \alpha\right)$ with $D \cap \mathbb{R}=T^{\prime}$ which is mapped diffeomorphically onto $D(T ; \alpha)$ by $F$.

In fact, there is generalization of this lemma to the situation in which $G=F^{-1}$ is not defined on the entire region $\mathbb{C}_{T}^{\prime}$ :

Lemma 2.3 (Schwarz) For each $\theta_{0} \in(0, \pi)$ there exists $\delta>0$ and $K>0$ with the following properties. Let $G$ be a univalent map defined on the unit disc $D_{1}(0)$, mapping the real diameter $D_{1}(0) \cap \mathbb{R}$ into the real line. Let $T$ be an interval on the real line containing the origin with $|T|<\delta$ and $G(T)=T^{\prime}$. Then for each $\theta \in\left(0, \theta_{0}\right)$,

$$
G(D(T ; \pi-\theta)) \subset D\left(T^{\prime} ; \pi-\theta(1+K|T|)\right) .
$$

Proof: See Lemma VI.5.2 in [MS]. 


\section{The proof of Theorem A in the Epstein case}

Let us first prove Theorem A for Epstein maps. Here we say that a map $g: \cup I_{2} \rightarrow I$ from the class $\mathbf{A C}$ is in the more restrictive class of Epstein maps $\mathbf{E C}$ (cf. [Su]) if

1. for every $i \neq 0$, the map $g: I_{i} \rightarrow I$ is a diffeomorphism such that $g^{-1}: I \rightarrow I_{i}$ has a univalent extension to the slit complex plane $\mathbb{C}_{I}$,

2. one can decompose the map $g: I_{0} \rightarrow I$ as $g(x)=g(0)+[h(x)]^{\ell}$, where $\ell \geq 3$ is an odd integer, and $h: I_{0} \rightarrow I_{0}^{\prime}$ is a diffeomorphism with $h(0)=0$, such that $h^{-1}: I_{0}^{\prime} \rightarrow I_{0}$ has a univalent extension to $\mathbb{C}_{I_{0}^{\prime}}$.

We remark that $g$ from 1 ) and $h$ from 2 ) in the above definition increase cross-ratios. Indeed, since $g^{-1}$ and $h^{-1}$ extend analytically to the slit complex plane and since the Poincaré metric on a disc restricted to its diameter corresponds to cross-ratios, the remark follows from the Schwarz lemma.

For each $\epsilon$, take $\Delta(\epsilon)$ as in Lemma 2.1. Since $\left|U_{n}\right| \rightarrow 0$ as $n \rightarrow \infty$, for each $\varepsilon>0$ we shall only consider integers $n$ which are so large that $\left|U_{n}\right|<\Delta(\epsilon)$.

Lemma 3.1 There exists $\epsilon_{0}>0$ such that if for some $\epsilon \in\left(0, \epsilon_{0}\right)$ there exist infinitely many n's for which

$$
\max \left(\left|\partial_{l} U_{n}-c\right|,\left|\partial_{r} U_{n}-c\right|\right)<(1+\epsilon) \cdot \operatorname{dist}\left(\partial U_{n-1}, c\right)
$$

then g has complex bounds.

Proof: Assume that the above inequality is satisfied. To be more concrete, assume that

$$
\left|\partial_{l} U_{n}-c\right| \leq\left|\partial_{r} U_{n}-c\right|<(1+\epsilon) \cdot\left|\partial_{l} U_{n-1}-c\right|
$$

Consider two cases.

I. $U_{n}$ is well inside $U_{n-1}$. Then we take $\Omega=D_{*}\left(U_{n-1}\right)$ as the range of the polynomiallike map. Consider the component of the domain of the first return map to $U_{n-1}$ which intersects $U_{n}^{g}$ (we shall use this notation for the interval $g\left(U_{n}\right)$ ). By Schwarz and because $g$ is in Epstein class this component is inside $D_{*}\left(U_{n}^{g}\right)$. If $\epsilon$ would be equal to zero, then because we are assuming in this case that $U_{n}$ is well inside $U_{n-1}$, $g^{-1}\left(D_{*}\left(U_{n}^{g}\right)\right)$ is compactly contained in $D_{*}\left(U_{n-1}\right)$. Provided $\epsilon \in\left(0, \epsilon_{0}\right)$ and we choose $\epsilon_{0}$ sufficiently small, the same is still true provided (3.1) holds. Note that the modulus of $D_{*}\left(U_{n-1}\right) \backslash g^{-1}\left(D_{*}\left(U_{n}^{g}\right)\right)$ is bounded from below by a positive constant which does not depend on $n$. Again by Schwarz the non-central domains are mapped univalently inside $D_{*}\left(U_{n-1}\right)$.

II. $U_{n}$ is not well inside $U_{n-1}$. According to Theorem 2.1, then the previous return is central (actually, many of the previous returns are central if $U_{n}$ is not well inside $U_{n-1}$ ). Hence, $U_{n}$ and $U_{n-1}$ are two consecutive intervals in a cascade of central returns. Then, on the one hand there exists a first interval $U_{n-k}$ of this cascade, so that $S_{n}=S_{n-k}$ and $U_{n-k}$ is well inside $U_{n-k-1}$. On the other hand, let $U_{m}$ (with $m \geq n$ ) be the last 
interval of this cascade, so that $S_{m}=S_{n}$ and the map $g^{S_{m}}: U_{m} \rightarrow U_{m-1}$ is non-central. So we have

$$
S_{m+1}>S:=S_{m}=\ldots=S_{n}=\ldots=S_{n-k+1}=S_{n-k}>S_{n-k-1}
$$

and the following situation (where $*$ are points of the form $g^{i S}(c)$ )

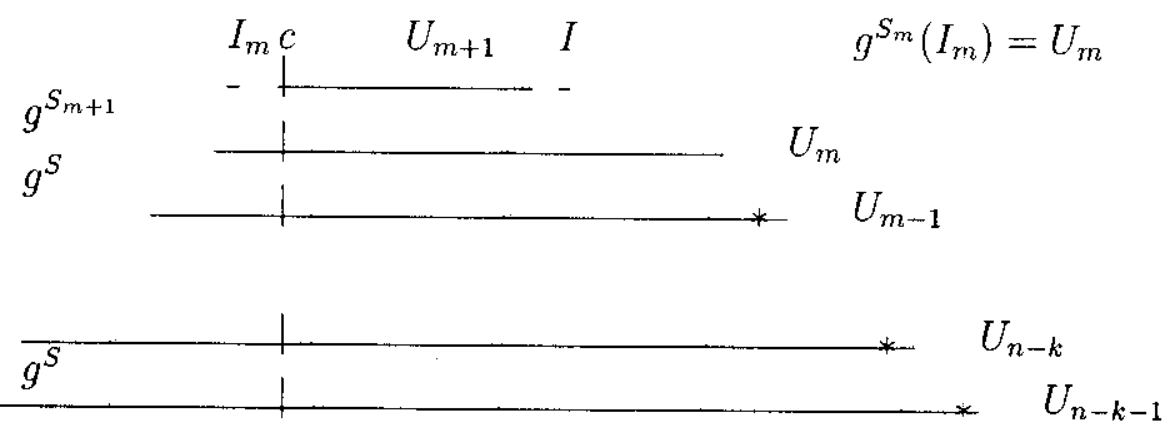

where $S_{m+1}$ and $S=S_{m}=\ldots=S_{n-k}$ are the first return times of $c$ to $U_{m}$ respectively $U_{m-1}$ and $I_{m}$ is the component of the first return to $U_{m}$. which is mapped by $g^{S_{m}}$ onto $U_{m}$ (which is the left of $c$ if $f^{S_{m}}(c) \in r\left(U_{m-1} \backslash U_{m}\right)$ and to the right otherwise). Proposition 2.2 implies that there is an interval between $U_{m}^{g}$ and $U_{m+1}^{g}$ (i.e., contained in the former interval and containing the latter one) which is mapped by $g^{S_{m+1-1}}$ diffeomorphically onto $U_{n-k-1}$. Let $U_{*}$ be the smallest symmetric interval which contains $U_{m}$. Since $U_{n-1}$ is well inside $U_{n-k-1}$, provided $\epsilon$ is small, (3.1) implies that $U_{*}$ is well inside $U_{n-k-1}$. Therefore, as we remarked before, there exists an interval $U$ with $U_{m} \supset U \supset U_{m+1}$ and so that $g^{S_{m+1-1}}$ maps $g(U)$ diffeomorphically onto $U_{*}$. Since $U_{*}$ is well inside $U_{n-k-1}$, the interval $U$ is well inside $U_{m}$. Take as the range $\Omega$ of the desired quasi-polynomial-like map the disc $D_{*}\left(U_{*}\right)$ with two slits (cf. the proof of Lemma 3.6):

$$
\Omega=D_{*}\left(U_{*}\right) \backslash\left(U_{*} \backslash U_{m}\right)
$$

We put the slits in $D_{*}\left(U_{*}\right)$ because we want our quasi-polynomial-like map restricted to the real line to coincide with the first return map to $\Omega$ along the postcritical set (and so the return time of $c$ to $\Omega$ is equal to $S_{m+1}$ ). Consider the first return map to $\Omega$ along the postcritical set. By Schwarz the $g$-image of the central domain $\Omega_{0}$ of the first return map to $\Omega$ is contained in $D_{*}(g(U))$. Because $D_{*}\left(U_{*}\right)$ is a round disc centered at $c=0$, and because $U$ is well inside $U_{m} \subset U_{*}$, we obtain that $\Omega_{0}$ is compactly contained in the range $\Omega$ (and we obtain a lower bound for the modulus of $\Omega \backslash \Omega_{0}$ which does not depend on $n$ ). Next consider the non-central components of the first return map. Take a non-central real domain $I$ of the first return map $R_{U_{m}}$ to $U_{m}$ and let $s$ be so that $\left.R_{U_{m}}\right|_{I}=g^{s}$. If $s>S_{m}\left(=S_{n}=S_{n-k}\right)$, then there is a diffeomorphic extension $g^{s}: \hat{I} \rightarrow U_{*}$ of $\left.g^{s}\right|_{I}$, and the pullback of $\Omega$ by $g^{s}$ is contained in $D_{*}(\hat{I})$ and hence is compactly contained in $\Omega$ (here we use similar arguments as above). Consider the remaining branch $g^{S_{m}}: I_{m} \rightarrow U_{m}$ of the first return map to $U_{m}$. It has a diffeomorphic extension $g^{S_{m}-1}: \hat{I}_{m}^{g} \rightarrow U_{*}$ only from an interval $\hat{I}_{m}^{g} \subset U_{*}^{g}$ (so near the critical value), and the pullback of $\Omega$ by $g^{S_{m}-1}$ is contained in $D_{m}^{g}:=D_{*}\left(\hat{I}_{m}^{g}\right) \backslash\left(\hat{I}_{m}^{g} \backslash I_{m}^{g}\right)$ 
(with $g(c) \in\left(\hat{I}_{m}^{g} \backslash I_{m}^{g}\right)$ ). But since $\Omega$ is a disc centered at $c$ (with two slits), the branch of the map $g^{-1}$ corresponding to $g^{-1}: I_{m}^{g} \rightarrow I_{m}$, takes $D_{m}^{g}$ onto some $D_{m}$, which is inside $\Omega$, but possibly not compactly inside: the boundaries of $\Omega$ and $D_{m}$ can coincide in a subset of the slits $U_{*} \backslash U_{m}$. Thus we obtain a quasi-polynomial-like mapping (the definition is given below the statement of Theorem A).

If we are in the situation of the previous lemma, then Theorem A follows. So we may and will assume that for each $\epsilon \in\left(0, \epsilon_{0}\right)$ there exists $N$ so that for all $n \geq N$,

$$
\max \left(\left|\partial_{l} U_{n}-c\right|,\left|\partial_{r} U_{n}-c\right|\right)>(1+\epsilon) \cdot \operatorname{dist}\left(\partial U_{n-1}, c\right)
$$

i.e., the larger component of $U_{n} \backslash\{c\}$ is a definite amount longer than the shortest component of $U_{n-1} \backslash\{c\}$ (if $\epsilon=0$ then it is already as least as long). Since $U_{n} \subset U_{n-1}$ this means that for each $\epsilon \in\left(0, \epsilon_{0}\right)$ there exists $N(\epsilon)$ so that either for all $n \geq N(\epsilon)$ $(1+\epsilon)\left|\partial_{l} U_{n-1}-c\right|<\left|\partial_{r} U_{n}-c\right|$ or for all $n \geq N(\epsilon)(1+\epsilon)\left|\partial_{r} U_{n-1}-c\right|<\left|\partial_{l} U_{n}-c\right|$. In order to be specific, we will from now on make the following

STANDING ASSUMPTION: $(1+\epsilon) \cdot\left|\partial_{l} U_{n-1}-c\right|<\left|\partial_{r} U_{n}-c\right|$ for all $n \geq N(\epsilon)$.

In particular, we assume that for each $n \geq N(\epsilon)$ the right component of $U_{n} \backslash\{c\}$ is a definite amount longer than its left component. This means that for $n \geq N(\epsilon)$,

Lemma 3.2 If $J \subset T$ are intervals such that

$$
T \subset U_{n-1}, J \subset r\left(U_{n-1} \backslash U_{n}\right)
$$

then we can pass through the critical point without loosing on cross-ratio:

$$
C^{-1}(T, J) \geq C^{-1}(g(T), g(J)) .
$$

Proof: Follows from Lemma 2.1 and the standing assumption.

Lemma 3.3 Assume that the standing assumption holds. Then there are infinitely many $n$ 's such that $g^{S_{n}}(c)<c$.

Proof: By the contrary, assume that $g^{S_{n}}(c)>c$ for all $n \geq N$. It is enough to show that $C^{-1}\left(U_{n}, U_{n+1}\right) \rightarrow \infty$ as $n \rightarrow \infty$ provided $S_{n}>S_{n-1}$ because it would contradict the standing assumption. Consider $U_{n} \subset U_{n-1}$ and assume that $S_{n}>S_{n-1}$. Since $g^{S_{n-1}}(c)>c, g$ is orientation preserving on each branch and $S_{n}>S_{n-1}$ one has $g^{S_{n-1}}\left(U_{n}\right) \subset r\left(U_{n-2} \backslash U_{n-1}\right)$ and by definition $g^{S_{n-1}}\left(U_{n-1}\right)=U_{n-2}$. Next apply $g^{S_{n-2}}=g^{S_{n-2-1}} \circ g$. Lemma 3.2 and the standing assumption give us

$$
\begin{gathered}
C^{-1}\left(U_{n-2}, g^{S_{n-1}}\left(U_{n}\right)\right) \geq C^{-1}\left(g^{S_{n-2}}\left(U_{n-2}\right), g^{S_{n-2}+S_{n-1}}\left(U_{n}\right)\right)= \\
=C^{-1}\left(U_{n-3}, g^{S_{n-2}+S_{n-1}}\left(U_{n}\right)\right) .
\end{gathered}
$$


Again $g^{S_{n-1}+S_{n-2}}\left(U_{n}\right) \subset r\left(U_{n-3} \backslash U_{n-2}\right)$ and by definition $g^{S_{n-1}+S_{n-2}}\left(U_{n-1}\right)=U_{n-3}$. Hence $S_{n-1}+S_{n-2}<S_{n}$ because $g^{S_{n}}\left(U_{n}\right)=U_{n-1}$. Continuing in this way we apply next $g^{S_{n-3}}, g^{S_{n-4}}$ and so on (using each time Lemma 3.2). If we write $t(n)=S_{n-1}+\ldots+S_{N}$ then we get that $t(n)<S_{n}$, that $g^{t(n)}$ maps $U_{n-1}$ homeomorphicaliy onto $U_{N-1}$ and that

$$
C^{-1}\left(U_{n-2}, g^{S_{n-1}}\left(U_{n}\right)\right) \geq C^{-1}\left(U_{N-1}, g^{t(n)}\left(U_{n}\right)\right) .
$$

Moreover, $\left|g^{t(n)}\left(U_{n}\right)\right| \rightarrow 0$ because $g^{S_{n}}\left(U_{n}\right)=U_{n-1}$ tends to zero in length (here we can use the contraction principle, see for example [MS], since $g$ has no wandering intervals). Moreover, $g^{t(n)}\left(U_{n}\right)$ does not tend to $\partial U_{N-1}$ because $\omega(c)$ is assumed to be minimal. It follows that $C^{-1}\left(U_{N-1}, g^{t(n)}\left(U_{n}\right)\right) \rightarrow \infty$. Therefore, $C^{-1}\left(U_{n-1}^{g}, g\left(U_{n}\right)\right) \geq$ $C^{-1}\left(U_{n-2}, g^{S_{n-1}}\left(U_{n}\right)\right) \rightarrow \infty$, and we are done.

Let us from now on assume that $g^{S_{n}}(c)<c$ for infinitely many $n$. So consider the (infinite) collection of integers

$$
\mathcal{S}=\left\{n ; g^{S_{n}}(c)<c \text { and } S_{n+1}>S_{n}\right\} .
$$

Note that for $n \in \mathcal{S}$ one cannot have that $g^{S_{n}}: U_{n} \rightarrow U_{n-1}$ is central, but it is possible that $g^{S_{n-1}}: U_{n-1} \rightarrow U_{n-2}$ is central. In other words, if there are central intervals then $U_{n}$ is always the last (smallest) interval in a chain of central intervals

$$
U_{n} \subset U_{n-1} \subset \ldots \subset U_{n-k(n)}
$$

with

$$
S_{n+1}>S_{n}=S_{n-1}=\ldots=S_{n-k(n)}>S_{n-k(n)-1} .
$$

Here $k(n) \geq 0$, and $k(n)=0$ if and only if $g^{S_{n-1}}: U_{n-1} \rightarrow U_{n-2}$ is again not central. Let $n_{1}<n_{2}<n_{3}<\ldots$ be the elements of this collection $\mathcal{S}$. Below we draw the situation when $k\left(n_{i}\right)=0$.
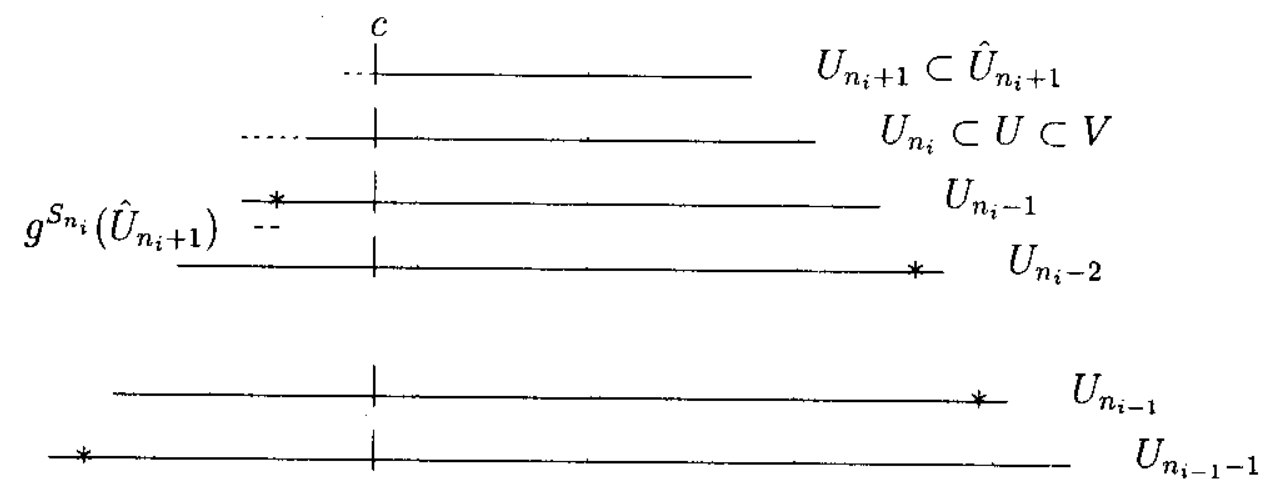

Let

$$
V=\left[\partial_{l} U_{n_{i}-k\left(n_{i}\right)-1}, \partial_{r} U_{n_{i}}\right] .
$$

It will also be useful to define an interval $\hat{U}_{n_{i}+1}$ containing $c$ between $U_{n_{i}+1}$ and $U_{n_{i}}$ with $\partial_{r} \hat{U}_{n_{i}+1}=\partial_{r} U_{n_{i}+1}$ and so that

$$
g^{S_{n_{i}+1}}: \hat{U}_{n_{i}+1} \rightarrow\left[\partial_{l} U_{n_{i}-k\left(n_{i}\right)-1}, \partial_{r} U_{n_{i}}\right]=V
$$


is a homeomorphism. By Proposition 2.2, $g^{S_{n_{i}+1}-1}: \hat{U}_{n_{i}+1}^{g} \rightarrow V$ is a diffcomorphism.

\section{Lemma 3.4}

$$
\begin{gathered}
C^{-1}\left(V^{g}, \hat{U}_{n_{i}+1}^{g}\right)=C^{-1}\left(\left[\partial_{l} U_{n_{i}-k\left(n_{i}\right)-1}^{g}, \partial_{r} U_{n_{i}}^{g}\right], \hat{U}_{n_{i}+1}^{g}\right) \geq \\
\geq C^{-1}\left(\left[\partial_{l} U_{n_{i-1}-1}, \partial_{r} U_{n_{i}-1}\right], g^{S_{n_{i}}}\left(\hat{U}_{n_{i}+1}\right)\right) .
\end{gathered}
$$

Proof: Write $\hat{U}=\hat{U}_{n_{i}+1}$ and choose $U$ between $U_{n_{i}}$ and $V$ (such that $U, U_{r_{i}}, V$ all have the same right end point) so that $U$ is mapped homeomorphically by $g^{S_{n_{i}}}$ onto $\left[\partial_{l} U_{n_{i-1}-1}, \partial_{r} U_{n_{i}-1}\right]$. This means that

$$
g^{S_{n_{i}-1}}: \hat{U}^{g} \subset U^{g} \rightarrow g^{S_{n_{i}}}(\hat{U}) \subset\left[\partial_{l} U_{n_{i-1}-1}, \partial_{r} U_{n_{i-1}}\right]
$$

is a homeomorphism between these pairs of intervals. It is enough to show that we do not have loss of cross-ratios for this map, because obviously the left-hand side of the inequality in the statement of the lemma is larger than $C^{-1}\left(U^{g}, \hat{U}^{g}\right)$. Note that $g^{S_{n_{i}-1}}$ is a diffeomorphism restricted to $\hat{U}^{g}$ and also restricted to the right component of $U^{g} \backslash \hat{U}^{g}$ (because these sets are contained in $U_{n_{i}}$ ). So we need to show that each time some iterate (up to tine $S=S_{n_{i}}$ ) of the left component of $U^{g} \backslash \hat{U}^{g}$ meets the critical point we are in the situation described by Lemma 3.2.

The first time $c$ enters $U_{n_{i}-k\left(n_{i}\right)-1}$ is at time

$$
S=S_{n_{i}}=\ldots=S_{n_{i}-k\left(n_{i}\right)}\left(>S_{n_{i}-k\left(n_{i}\right)-1} \geq \ldots \geq S_{n_{i-1}}\right) .
$$

So all iterates of $\hat{U}$ up to time $S=S_{n_{i}}$ are outside $U_{n_{i}-k\left(n_{i}\right)-1}$. Moreover, they do not intersect $\partial U_{t}$ when $t \leq n_{i}$ because $g^{S_{n_{i}}}(\hat{U}) \subset U_{n_{i}-1}$. Let us take $j<S_{n_{i}}$ and show that either $g$ is a diffeomorphism on $g^{j}(U)$ or we are in the situation of Lemma 3.2 for $J=g^{j}(\hat{U}) \subset g^{j}(U)=T$. If $g^{j}(\hat{U})$ is outside $U_{n_{i-1}}$ then $g^{j}(U)$ is also outside $U_{n_{i-1}}$ (and so $g$ is a diffeomorphism on $g^{j}(U)$ ); indeed boundary points of $U_{n_{i-1}}$ are never mapped inside $U_{n_{i-1}}$. If $g^{j}(\hat{U})$ is in $U_{n_{i-1}}$ but to the left of $c$, then $g^{j}(U)$ also does not contain $c$ (because $g$ preserves orientation and $g^{S_{n_{i}-1}}$ is a diffeomorphism restricted to the right component of $\left.U^{g} \backslash \hat{U}^{g}\right)$, and again $g$ is a diffeomorphism on $g^{j}(U)$. So the only situation we have to consider is when $g^{j}(\hat{U}) \subset r\left(U_{n_{i-1}} \backslash U_{n_{i}-k\left(n_{i}\right)-1}\right)$. In this case $g^{j}(\hat{U})$ is contained in one of the intervals $r\left(U_{n_{i}-s} \backslash U_{n_{i}-s+1}\right)$ where $n_{i-1} \leq n_{i}-s<n_{i}-k\left(n_{i}\right)-1$. We claim that if

$$
g^{j}(\hat{U}) \subset r\left(U_{n_{i}-s} \backslash U_{n_{i}-s+1}\right)
$$

(with $s$ as above) then $g^{j}(U)$ is contained in $U_{n_{i}-s}$. So let us assume by contradiction that $j<S_{n_{i}}$ is the maximal integer such that there exists $s$ as in (3.2) such that $g^{j}(U)$ contains a boundary point of $U_{n_{i}-s}$. It is impossible that $n_{i}-s=n_{i-1}$ because if $g^{j}(U)$ contains a boundary point of $U_{n_{i}-s}$ then $g^{S}(U)$ would contain the left boundary of $U_{n_{i-1}-1}$ which is not the case. If $n_{i}-s>n_{i-1}$ then $g^{S_{n_{i}-s}}$ is the first iterate which maps $g^{j}(\hat{U})$ inside $U_{n_{i}-s-1}$. It also maps $g^{j}(\hat{U})$ into $r\left(U_{n_{i}-s-1} \backslash U_{n_{i}-s}\right)$ (because $n_{i}-s<n_{i}-k\left(n_{i}\right)-1$ and by the definition of $\mathcal{S}$ ). From this it follows that $j$ is not maximal, and so by contradiction the claim is proved. But from the claim it follows 
that for each $j$ with (3.2), we are in the situation of Lemma 3.2, and thus the lemma follows.

Lemma 3.5 For each $\epsilon$ from the standing assumption there are $\delta>0$ and infinitely many $n_{i} \in \mathcal{S}$ for which

$$
C^{-1}\left(\left[\partial_{l} U_{n_{i}-k\left(n_{i}\right)-1}^{g}, \partial_{r} U_{n_{i}}^{g}\right], \hat{U}_{n_{i}+1}^{g}\right) \geq 1+\delta
$$

Proof: Fix such $\epsilon$. By the previous lemma it suffices to prove that there are $\delta>0$ and infinitely many $n_{i} \in \mathcal{S}$ such that

$$
C^{-1}\left(\left[\partial_{l} U_{n_{i-1}-1}, \partial_{r} U_{n_{i}-1}\right], g^{S_{n_{i}}}\left(\hat{U}_{n_{i}+1}\right)\right) \geq 1+\delta .
$$

Moreover, since $g^{S_{n_{i}}}\left(\hat{U}_{n_{i}+1}\right) \subset l\left(U_{n_{i}-1} \backslash U_{n_{i}}\right)$, the left hand side in the previous inequality is bounded from below by

$$
C^{-1}\left(\left[\partial_{l} U_{n_{i-1}-1}, \partial_{r} U_{n_{i}-1}\right], l\left(U_{n_{i}-1} \backslash U_{n_{i}}\right)\right)
$$

Normalize so that $c=0$ and write

$$
U_{n}=\left[-x_{n}, y_{n}\right]
$$

where by our standing assumption we have $0<(1+\epsilon) x_{n}<y_{n+1}<y_{n}$ provided $\epsilon \in\left(0, \epsilon_{0}\right)$. Then

$$
\begin{aligned}
& C^{-1}\left(\left[\partial_{l} U_{n_{i-1}-1}, \partial_{r} U_{n_{i}-1}\right], l\left(U_{n_{i}-1} \backslash U_{n_{i}}\right)\right)=\frac{\left(x_{n_{i-1}-1}-x_{n_{i}-1}\right)\left(y_{n_{i}-1}+x_{n_{i}}\right)}{\left(y_{n_{i}-1}+x_{n_{i-1}-1}\right)\left(x_{n_{i}-1}-x_{n_{i}}\right)} \geq \\
& \frac{\left(x_{n_{i-1}-1}-x_{n_{i}-1}\right)\left((1+\epsilon) x_{n_{i}-1}+x_{n_{i}}\right)}{\left((1+\epsilon) x_{n_{i}-1}+x_{n_{i-1}-1}\right)\left(x_{n_{i}-1}-x_{n_{i}}\right)}=\frac{\left(\lambda_{n_{i}}^{\prime}-1\right)}{\left((1+\epsilon)+\lambda_{n_{i}}^{\prime}\right)} \frac{\left((1+\epsilon) \lambda_{n_{i}}+1\right)}{\left(\lambda_{n_{i}}-1\right)},
\end{aligned}
$$

where we denote

$$
\lambda_{n_{i}}=x_{n_{i}-1} / x_{n_{i}}
$$

and

$$
\lambda_{n_{i}}^{\prime}=x_{n_{i-1}-1} / x_{n_{i}-1} .
$$

Take any $\kappa>0$. Assume first that for all $i$ sufficiently large, $\lambda_{n_{i}} \geq(1+\kappa) \lambda_{n_{i}}^{\prime}$. Observe that

$$
\lambda_{n_{i}}^{\prime}=x_{n_{i-1}-1} / x_{n_{i-1}} \geq x_{n_{i-1}-1} / x_{n_{i-1}}=\lambda_{n_{i-1}} .
$$

Hence we would have $\lambda_{n_{i}} \geq(1+\kappa) \lambda_{n_{i-1}}$ for every $i$ large. It implies $\lambda_{n_{i}} \rightarrow \infty$, and, together with (3.4), $\lambda_{n_{i}}^{\prime} \rightarrow \infty$. But then the expression in (3.3) would be at least $1+\epsilon / 2$, for all $n_{i}$ large enough, which proves the statement in the considered case with $\delta=\epsilon / 2$. 
Assume now that for some fixed $i, \lambda_{n_{i}} \leq(1+\kappa) \lambda_{n_{i}}^{\prime}$. Denote $t=\lambda_{n_{i}}^{\prime}$. Then the expression in (3.3) is bounded from below by the function

$$
A(\kappa, t)=\frac{(t-1)}{((1+\epsilon)+t)} \frac{((1+\epsilon)(1+\kappa) t+1)}{((1+\kappa) t-1)},
$$

which one can rewrite as

$$
(1+\epsilon)+(2+\epsilon) \frac{(\epsilon-((1+\epsilon)(1+\kappa)-1) t)}{((1+\epsilon)+t)((1+\kappa) t-1)} .
$$

It is easy to see that the latter expression is increasing on $t>1$. The real bounds imply that there exists $C>1$ so that $\lambda_{n_{i}}^{\prime} \geq C>1$ for all $i$. Hence, one can assume that $t \geq C$ and $A(\kappa, t) \geq A(\kappa, C)$. On the other hand, $A(\kappa, C) \rightarrow 1+2 \delta$ as $\kappa \rightarrow 0$, where we denote $\delta=0.5 \epsilon(C-1) /(1+\epsilon+C)>0$. It follows that there exists $\kappa>0$ dependent only on $C$ and $\epsilon$ such that the expression in (3.3) is again at least $1+\delta$ provided $\lambda_{n_{i}} \leq(1+\kappa) \lambda_{n_{i}}^{\prime}$ for a given $i$.

Let us now show that the situation of the previous lemma leads to complex bounds. Let $n=n_{i}$ and $k=k\left(n_{i}\right)$. It follows immediately from the previous lemma that

$\left(^{*}\right)$ the length of each component of $\left[\partial_{l} U_{n-k-1}^{g}, \partial_{r} U_{n}^{g}\right] \backslash\left\{c_{1}\right\}$ is bigger than $(1+\delta)\left|\hat{U}_{n+1}^{g}\right|$, where $\delta>0$ does not depend on $n=n_{i}$.

Let us choose (and then fix) $\Delta>0$ dependent only on $\delta$ as follows. Let

$$
V_{n-k-1}(\Delta) \subset\left[\partial_{l} U_{n-k-1}, \partial_{r} U_{n}\right]
$$

be the interval obtained by removing from the left side of the interval $\left[\partial_{l} U_{n-k-1}, \partial_{r} U_{n}\right]$ its $\Delta$-part, i.e. an interval of length $\Delta \cdot\left|\partial_{r} U_{n}-\partial_{l} U_{n-k-1}\right|$. We claim that for $\Delta>0$ is sufficiently small,

$\left.{ }^{* *}\right)$ the length of each component of $V_{n-k-1}^{g}(\Delta) \backslash\left\{c_{1}\right\}$ is larger than $(1+$ $\delta / 2)\left|\hat{U}_{n+1}^{g}\right|$. Moreover, $V_{n-k-1}(\Delta)$ contains the left end of $U_{n-k}$ and $V_{n-k-1}(\Delta)$ is well inside $U_{n-k-1}$.

Let us show that this is indeed possible. Let $l$ and $r$ be lengths of the left and the right components of $\left[\partial_{l} U_{n-k-1}^{g}, \partial_{r} U_{n}^{g}\right] \backslash \hat{U}_{n+1}^{g}$. Since $U_{n}$ is well inside $U_{n-k-1}$, there is an absolute constant $C>0$ such that $l \geq C \cdot\left|U_{n}^{g}\right| \geq C\left(r+\left|\hat{U}_{n+1}^{g}\right|\right)$. From the previous lemma, $l, r>\left|\hat{U}_{n+1}^{g}\right|$. This and $l \geq C\left(r+\left|\hat{U}_{n+1}^{g}\right|\right)$, imply that one can choose $\Delta>0$ (dependent on $C$ and $\delta$ ) as in the first part of $\left(^{* *}\right)$. Now, the left component of $U_{n-k-1} \backslash V_{n-k-1}(\Delta)$ has the length at least $\Delta\left|V_{n-k-1}(\Delta)\right|$. The right component of $U_{n-k-1} \backslash V_{n-k-1}(\Delta)$ is also not small compared with $V_{n-k-1}(\Delta)$ because $U_{n-k}$ is well inside $U_{n-k-1}$ and by the standing assumption. This gives the second part of $\left({ }^{*} *^{\prime}\right)$. 
Lemma 3.6 Let $\delta>0$ and $\Delta$ be as above. Then for any $n=n_{i} \in \mathcal{S}, k=k\left(n_{i}\right)$ with

$$
C^{-1}\left(\left[\partial_{l} U_{n-k-1}^{g}, \partial_{r} U_{n}^{g}\right], \hat{U}_{n+1}^{g}\right) \geq 1+\delta
$$

the first return map to $U_{n}$ extends to a (quasi-)polynomial-like map with range

$$
\Omega=D_{*}(V) \backslash\left(V \backslash U_{n}\right)
$$

where $V$ is either $\left[-\partial_{r} U_{n}, \partial_{r} U_{n}\right]$ or $V_{n-k-1}(\Delta)$.

Proof: There are two cases:

I. There exists $\rho>0$ (not depending on $n$ ) such that the left component of $U_{n-k-1} \backslash$ $\{c\}$ is at least $(1+\rho)$ times as long as the right component of $U_{n} \backslash\{c\}$. In this case $U_{*}=\left(-\partial_{r} U_{n}, \partial_{r} U_{n}\right)$ is well-inside $U_{n-k-1}$. Now proceed as in case II of Lemma 3.1 by setting $\Omega=D_{*}\left(U_{*}\right) \backslash\left(U_{*} \backslash U_{n}\right)$ (and taking $m=n$ ).

II. The situation is not as in I. Then let $V=V_{n-k-1}(\Delta)$. Since $U_{n-k}$ is wellinside $U_{n-k-1}$, that the situation is not as in I means that we can assume that the left component of $U_{n-k} \backslash\{c\}$ is shorter than the right component of $U_{n} \backslash\{c\}$.

Consider the first return map to $U_{n}$, but extend each branch so that it maps onto $\Omega:=D_{*}(V) \backslash\left(V \backslash U_{n}\right)$. Let us first consider the central domain of the first return map to $U_{n}$ and its extension $g^{S_{n+1}}: \hat{U}(\Delta) \rightarrow V$. Let $g^{S_{n+1}}: D_{0} \rightarrow \Omega$ be the central branch. By Schwarz, some subset of $D_{*}\left(\hat{U}(\Delta)^{g}\right)$ is mapped diffeomorphically onto $D_{*}(V)$ by $g^{S_{n+1}-1}$. From $\left(^{* *}\right)$ it follows immediately that $g^{-1}\left(D_{*}\left(\hat{U}(\Delta)^{g}\right)\right) \subset D_{*}(V)$. To show that the modulus of $\Omega \backslash D_{0}$ is bounded from below by some universal positive constant, we still need to consider the real part of $D_{0}$ (because of the slits). Let $\tilde{U} \subset U_{n}$ be so that $g^{S_{n+1}-1}: \tilde{U}^{g} \rightarrow U_{n-k-1}$ is a diffeomorphism. Since $V$ is well inside $U_{n-k-1}\left(\right.$ by $\left({ }^{* *}\right)$ ), the interval $\hat{U}(\Delta)$ is well inside $\tilde{U}$, i.e. also well inside $U_{n}$. All this means that the modulus of $\Omega \backslash D_{0}$ is bounded away from zero. Let us now show that the non-central components are also contained in $\Omega$. Take a non-central domain $I$ of the first return map to $U_{n}$ so that the first return restricted to $I$ coincides with $g^{s}$. If $s>S_{n}$, then, since forward iterates of $\partial U_{i}$ never enter $U_{i}$, this branch of the first 'return' map from $U_{n+1}$ to $U_{n}$ extends diffeomorphically onto $U_{n-k-1}$ (and the extended domain is well inside $U_{n}$ ). By the lemma of Schwarz, the corresponding domain is inside $\Omega$. So let us consider the case that $s=S_{n}$. In this case $I$ is the non-central interval in $U_{n}$ which is mapped by $g^{s}$ to $U_{n}$, and one can extend the interval $I^{g}=g(I)$ to the left to an interval $\tilde{I}^{g}=g(\tilde{I})$ so that $g^{s-1}: I^{g} \rightarrow U_{n}$ extends to a diffeomorphism $g^{s-1}: \tilde{I}^{g} \rightarrow V=\left[\partial_{l} U_{n-k-1}(\Delta), \partial_{r} U_{n}\right]$. The extended domain $\tilde{I}$ is inside $J=\left[\partial_{l} U_{n-k}, \partial_{r} U_{n}\right]$. Since we are in Case II, the right component of $U_{n}^{g} \backslash\{c\}$ is longer than the left component of $\tilde{I}^{g} \backslash\{c\}$. Therefore we can apply the lemma from the appendix and get that the component of $g^{-1}\left(D_{*}\left(\tilde{I}^{g}\right)\right) \backslash \mathbb{R}^{-}$which contains $I$, is contained in $D_{*}(\tilde{I})$. So also the non-central components are contained in $\Omega$. Therefore also in this case we obtain a quasi-polynomial-like map. 


\section{Real bounds for smooth maps in the minimal case}

In this section, we will show that the real bounds of [Lc] hold without the negative Schwarzian condition. That is, in this section we shall prove Theorem 2.1 for any $C^{3}$ map $g$ which satisfies conditions 1-3 of the class AC (but is not necessary real analytic) and for which

- $g(x)=g(c)+[h(x)]^{l}$ in a neighbourhood of the critical point $c=0$, where $h$ is a $C^{3}$ local diffeomorphism and $h(0)=0$,

- $\omega(c)$ is minimal.

Note that we shall use Theorem 2.1 for maps $g \in \mathbf{A C}$ only if $\omega(c)$ is minimal. \$o fix such a map $g$.

The scheme of the proof is the following. In the first step, we prove a weaker form of Theorem 2.1, namely, the following a priori real bounds:

Lemma 4.1 There exist a positive number $C$, and a sequence $n_{i}$, so that for every $n=n_{i} \rightarrow \infty$, the length of every component of $U_{n-1} \backslash U_{n}$ is equal to at least $C \cdot\left|U_{n}\right|$.

This lemma implies that any branch $R_{U_{n_{2}}}: V \rightarrow U_{n_{i}}$ of the first return map has a diffeomorphic extension onto a definite neighbourhood of $U_{n_{i}}$ (namely, to $U_{n_{i}-1}$ ) provided $V$ and $U_{n_{i}}$ are disjoint.

In the second step we repeat the proof of Theorem 3.2 of [Ko] (replacing Lemma 3.2 .3 in [Ko] by Lemma 4.1). This shall imply

Lemma 4.2 Let $U=U_{n_{i}}$ be small enough, and let $g^{k}: W \rightarrow g(U)$ be a branch of the first return map to $g(U)$, such that $c \notin W$ and $W$ contains points of the postcritical set. Then the Schwarzian derivative $S\left(g^{k}\right)$ of $g^{k}$ on $W$ is negative.

In the third step we show how to go through the critical point arbitrary many times without loss of the cross-ratio under the conditions of Lemma 4 of [Le] or even stronger see Lemma 3.3, and Lemma 3.4. This will prove Theorem 2.1 for smooth maps as above.

In the final step, we will show that all real bounds from the previous section also hold for smooth maps $g$ as above.

Step 1: Firstly, it turns out that it is convenient to deal with another cross-ratio $B(T, J)$ instead of $C(T, J)$ defined by

$$
B(T, J)=\frac{|J||T|}{|L \cup J||R \cup J|}
$$

where $J \subset T$ are intervals and $L, R$ are connected components of $T \backslash J$. (Note that $1+C^{-1}=B^{-1}$ ) A collection of intervals $J_{i} \subset T_{i}, i=1, \ldots, n$, is said to satisfy the margins disjointness property (which was introduced in Section 2.3 of [Ko]) if for the components $L_{i}$ and $R_{i}$ of $T_{i} \backslash J_{i}$,

$$
L_{i} \cap L_{j} \neq \emptyset \text { implies } R_{i} \cap R_{j}=\emptyset, \text { for } 1 \leq i<j \leq n
$$


By Lemma 2.3.2 of [Ko], a consequence of this property is that

$$
\sum_{i=1}^{n}\left|L_{i}\right|\left|R_{i}\right| \leq 4 \max _{1 \leq i \leq n}\left|T_{i}\right|
$$

provided all $T_{i} \subset[-1,1]$.

The margins disjointness property is useful in view of the following statements (which imply that we can pull-back big space provided the margins disjointness property holds):

Theorem 4.1 [MS]

Let $f: N \rightarrow N$ be a $C^{3}$-map of an interval or a circle $N$ whose critical points are non-flat. Then there exists a constant $C_{1}=C_{1}(f)$ such that

$$
B(f(T), f(J)) \geq B(T, J) \exp \left(-C_{1}|L \| R|\right),
$$

where $f^{\prime}(x) \neq 0$ for all $x \in T$.

Proof: See Section IV.1 from [MS].

Lemma 4.3 Under the conditions of Lemma 2.1, but assuming only that $h$ is a local $C^{3}$-diffeomorphism, we have:

$$
B(F(T), F(J)) \geq B(T, J) \exp \left(-C_{1}|L||R|\right),
$$

where the constant $C_{1}$ depends only on the map $h$.

Proof: $F$ is a composition of $h$ and $x^{l}$. Then use Lemma 2.1 for the latter map and the previous Theorem 4.1 for $h$.

In order to obtain Lemma 4.1, we observe that the proofs of Propositions 1-3 in [Le] hold for the smooth $g$ as well, with the following changes.

The proofs in [Le] are based on combinatorial arguments (which of course do not depend on any analytic condition), the Koebe Principle (which also holds for smooth maps provided the intersection multiplicity is bounded [MS]), and the following Interval Adding Procedure: Given $g^{S_{n}}: U_{n} \rightarrow U_{n-1}$, let $t_{n}^{+}$be a maximal interval outside $U_{n}$ and to the right from $U_{n}$ with a common boundary point with $U_{n}$, so that $g^{S_{n}} \mid t_{n}^{+}$is defined and a diffeomorphism. The interval $t_{n}^{-}$is defined in the same way, but to the left of $U_{n}$. Let $t$ be either $t_{n}^{+}$or $t_{n}^{-}$. Note that one endpoint of $g^{S_{n}}(t)$ is in $\partial U_{n-1}$. If the other endpoint of $g^{S_{n}}(t)$ is an endpoint of the range $I$ of the map $g$, then we set $k=0$ and stop. Otherwise $t$ is the minimal interval, such that the boundary point of $t$ which is not in $\partial U_{n}$, is a critical point of $g^{S_{n}}$. In this case there exists $i, 1 \leq i \leq S_{n}-1$, so that $c$ lies at the boundary of the interval $g^{i}(t)$. So in this case we will apply the following operation (called "adding the interval $U_{n-1}$ "):

Since $g^{i}\left(U_{n}\right)$ is outside $U_{n-1}$ and $g^{i}(t)$ has $c$ in its boundary, the interval $g^{i}(t)$ contains also a boundary point of $U_{n-1}$. Hence, either $i+S_{n-1}>S_{n}$, or $g^{i+S_{n-1}}(t)$ 
contains a boundary point of $U_{n-2}$. Hence, again either $i+S_{n-1}+S_{n-2}>S_{n}$, or $g^{i+S_{n-1}+S_{n-2}}(t)$ contains a boundary point of $U_{n-3}$. We continue this process until we find $k \geq 1$ so that

$$
S:=i+S_{n-1}+\ldots+S_{n-k+1}<S_{n}
$$

while

$$
S+S_{n-k} \geq S_{n}
$$

Then $g^{S}(t)$ contains a boundary point of $U_{n-k}$. Let us extend the interval $g^{S}(t)$ so that it turns into the interval $\left[U_{n-1}, g^{S}(t)\right]$, and call this operation "adding the interval $U_{n-1}$ ". The boundary point of $t$ other than $\partial U_{n}$ lies in a component of $U_{m-1} \backslash U_{m}$, for some $m \leq n$. Since $\partial U_{m-1}$ are nice points, there exists a unique extension $\hat{t}=t_{n}^{ \pm}$ of $t$ inside $U_{m-1} \backslash U_{m}$ so that $g^{S}$ maps $\hat{t}$ onto the interval $\left[U_{n-1}, g^{S}(t)\right]$. At the end, if $g^{S_{n}}\left(\hat{t}_{n}^{ \pm}\right)$covers more than one interval of the form $g^{j}\left(U_{n-1}\right), 1 \leq j \leq S_{n-1}-1$, we shorten $\hat{t}_{n}^{ \pm}$so that $g^{S_{n}}\left(\hat{t}_{n}^{ \pm}\right)$is the minimal interval, which either covers one interval of such form, or such that $\left|g^{S_{n}}\left(\hat{t}_{n}^{ \pm}\right)\right|=\left|U_{n-1}\right|$. In other words, $g^{S_{n}}\left(t_{n}^{-} \cup U_{n} \cup t_{n}^{+}\right)=\hat{V}_{n-1}$, where $\hat{U}_{n-1}$ is said to be the minimal interval containing from each side of $U_{n-1}$ either its neighbour from the collection $g^{i}\left(U_{n-1}\right) .1 \leq i<S_{n-1}$, or a 1-neighbourhood of $U_{n-1}$ (i.e. of size $\left.\left|U_{n-1}\right|\right)$.

By construction, $g^{S_{n}} \mid t_{n}^{ \pm}$has at most $k_{n}^{ \pm}$critical points (see Lemma 1 of [Le] for detail). Now we shall prove the inequality of Lemma 1 of [Lc]:

Lemma 4.4 There exists $C>0$ which only depends only on $\ell$ and $\max \left(k_{n}^{-}, k_{n}^{+}\right)$so that for $n$ large enough

$$
\left|\hat{t}_{n}^{ \pm}\right| \geq C\left|U_{n}\right|
$$

Proof: Observe that the collection of intervals:

$$
g^{i}(J) \subset g^{i}(L \cup J \cup R), i=0, \ldots, S_{n}-1
$$

where $J=U_{n}, L, R=\hat{t}^{ \pm}$, satisfies the margins disjointness property (4.1). Indeed, otherwise one could find $1 \leq j \leq S_{n}-1$ with $g^{j}\left(t_{n}^{-}\right) \cap g^{S_{n}}\left(t_{n}^{-}\right) \neq \emptyset$ and $g^{j}\left(t_{n}^{+}\right) \cap g^{S_{n}}\left(t_{n}^{+}\right) \neq$ $\emptyset$. Because $g^{S_{n}}\left(U_{n}\right)=U_{n-1}$ and $g^{j}\left(U_{n}\right) \cap U_{n-1}=\emptyset$, then either $g^{j}\left(\left(t_{n}^{-}\right)\right.$or $g^{j}\left(t_{n}^{+}\right)$ contains a neighbourhood of $U_{n-1}$. If, say, $g^{j}\left(t_{n}^{+}\right) \supset \bar{U}_{n-1}$, then $g^{S_{n}}\left(t_{n}^{+}\right)$strictly contains $g^{S_{n-j}}\left(U_{n-1}\right)$, a contradiction with the choice of $t_{n}^{+}$.

The margins disjointness property implies that

$$
e_{n}:=\sum_{i=0}^{S_{n}}\left|g^{i}\left(t_{n}^{-}\right)\right|\left|g^{i}\left(t_{n}^{+}\right)\right| \leq 4 \max _{0 \leq i \leq S_{n}}\left|g^{i}(T)\right|
$$

where $T=t_{n}^{-} \cup U_{n} \cup t_{n}^{+}$. Here $e_{n} \rightarrow 0$, because the length of the last interval $g^{S_{n}}(T)$ tends to zero as $n \rightarrow \infty$ ( $g$ has no wandering intervals). From the Koebe principle and the shortest interval argument, there exists a positive constant $b_{0}<1$ (depending on $\ell$ only) such that

$$
B\left(\hat{U}_{n-1}, U_{n-1}\right) \leq b_{0} .
$$

Now pull $\hat{U}_{n-1}$ back to $T$. Let $0=j_{1}<\ldots<j_{r}<S_{n}$ be the moments such that $c \in g^{i}(T)$. As we know, $r \leq \max \left(k_{n}^{-}, k_{n}^{+}\right)$. Write $B_{i}=B\left(g^{i}(T), g^{i}\left(U_{n}\right)\right), 0 \leq i \leq S_{n}$. 
Using Theorem 4.1 one can write $B_{j_{r}+1} \leq B_{S_{n}} \exp \left(C_{1} \cdot e_{n}\right) \leq b_{1}<1$, where $b_{1}$ is universal (i.e., depends on $\ell$ only) provided $n$ is large enough. Passing from the critical value to the critical point, $B_{j_{r}} \leq b_{1}^{\prime}<1$, where $b_{1}^{\prime}$ depends only on $b_{1}$ (and on $\ell$, as always). Continuing in this way, we obtain finally $B_{0}=B\left(T, U_{n}\right) \leq b_{n}^{\prime}<1$, where $b_{r}^{\prime}$ depends only on the number $r \leq \max \left(k_{n}^{ \pm}\right)$. Therefore (4.2) is provec.

Thus Propositions 1-3 of [Le] hold for smooth maps. According to Propositions 1-2 of [Le], $U_{m}$ is well inside $U_{m-1}$ whenever either $U_{m-1}$ is the last int rval in a cascade of central returns, or $g^{S_{m-3}}(c)$ and $g^{S_{m-4}}(c)$ are on opposite sides of $c$. (We don't need Proposition 3 since $g$ preserves orientation. Lemma 5 of [Le] and its proof also remains unchanged.) Therefore, to complete the proof of Lemma 4.1, it is enough to have a weaker form of Lemma 4 of [Le]:

Lemma 4.5 Assume that $g^{S_{n}}: U_{n} \rightarrow U_{n-1}$ are not central and $g^{S_{n}}(c)>c$ for all $n$. Assume also that the Standing Assumption holds. Then $U_{n}$ is well inside $U_{n-1}$ for all $n \geq 1$.

Proof: To prove this we apply the Interval Adding Procedure. More precisely, given $n$, let $\hat{U}_{n-1}$ be as above. Let $T:=\hat{t}^{-} \cup U_{n} \cup \hat{t}^{+}$be an interval mapped onto $\hat{U}_{n-1}$ by $g^{S_{n}}$ (i.e., $\hat{t}^{ \pm}=\hat{t}_{n}^{ \pm}$). We observe that because of the assumptions made in the lemma

- $\hat{t}^{+}$is inside the right component of $U_{n-1} \backslash U_{n}$ (indeed, $g^{S_{n-2}}\left(U_{n-1}\right) \subset r\left(U_{n-3} \backslash U_{n-2}\right)$ and $g^{S_{n}}\left(r\left(U_{n-1} \backslash U_{n}\right)\right)$ covers $\left.r\left(U_{n-3} \backslash U_{n-1}\right)\right)$, and, therefore $g^{S_{n}}: \hat{t}^{+} \rightarrow \hat{U}_{n-1}$ has at most one critical point.

- $\hat{t}^{-}$is inside the left component of $U_{n-1} \backslash U_{n}$ (because $g^{S_{n-1}}\left(l\left(U_{n-1} \backslash U_{n}\right)\right) \supset U_{n-1}$, i.e., $g^{S_{n}}$ is not diffeomorphic on $l\left(U_{n-1} \backslash U_{n}\right)$ ). Moreover, each time an iterate of $\hat{t}^{-}$passes through the critical point, more than half of this iterate of $\hat{t}^{-}$lies to the right of $c$ (as required in order to apply Lemmas 2.1 and 4.3). This follows from the construction of $t^{ \pm}: g^{S}\left(\hat{t}^{-}\right)$contains an end point of $U_{n-k}$ and $\partial_{l} g^{S}\left(\hat{t}^{-}\right) \geq \partial_{l} U_{n-1}$ (see the Interval Adding Procedure).

Because of the latter observation, one can ignore that $g^{i}\left(\hat{t}^{-}\right)$covers $c$ from time to time, and apply the proof of (4.2) where we set $r=2$. We therefore obtain, that $B\left(U_{n-1}, U_{n}\right) \leq B\left(T, U_{n}\right) \leq b^{\prime}$, where $b^{\prime}<1$ depends on $b_{0}$ (and $\ell$ ) i.e. depends finally on $\ell$ only.

Thus we have proved (for the $C^{3}$-map g) Propositions 1-2 of [Le], Lemma 4.5 (instead of Lemma 4 of [Le]), and Lemma 5 of [Le]. As we have noticed it implies Lemma 4.1.

Step 2: Copying Sections 3.3-3.4 of [Ko], Lemma 4.2 also holds. Note that we can apply a theorem of Mañé (see Theorem 2.2) because $\omega(c)$ stays away from the parabolic periodic orbits of $g$ (of which there are at most finitely many, see [MS] and Proposition 2.1). 
Step 3: At last, let us prove Lemma 4 of [Le], or even stronger Lemma 3.3. We repeat its proof as follows (cf. proof of Theorem 3.1 of $[\mathrm{Ko}]$ ). Fix an interval $T_{0}=U_{n_{i_{0}}}$ with $n_{i_{0}}$ so large that we can apply Lemma 4.2. Now, in the proof of Lemma 3.3, each time we apply $g^{S_{r}}$ to the interval $T:=U_{r}=g^{S_{n-1}+\ldots+S_{r+1}}\left(U_{n-1}\right)$ (with the interval $J:=g^{S_{n-1}+\ldots+S_{r+1}}\left(U_{n}\right)$ inside, so that $\left.T=L \cup J \cup R\right)$, we divide the orbit iato the first $M$ iterates and the remaining iterates. To be more precise, let $M<S_{r}$ be the maximal integer so that $g^{M-1}(T) \subset T_{0}$. Then, by Lemmas $4.2-4.3, B\left(g^{M}(T), g^{M^{\prime}}(J)\right) \geq$ $B(g(T), g(J)) \geq B(T, J) \exp \left(-C_{1} \cdot|L| \cdot|R|\right)$. The iterates $g^{j}(T), j=M, \ldots, S_{r}-1$, from $M$ to $S_{r}-1$ are outside $T_{0}$ and stay away from a fixed neighbourhood of all parabolic periodic orbits of $g$, because $\omega(c)$ is minimal. Hence, we can use the theorem of Mañé (see 2.2) to obtain:

$$
\begin{gathered}
B\left(g^{S_{r}}(T), g^{S_{r}}(J)\right) \geq B\left(g^{M}(T), g^{M}(J)\right) \exp \left(-C_{1} \sum_{i=M}^{S_{r}-1}\left|g^{i}(L)\right|\left|g^{i}(R)\right|\right) \geq \\
B\left(g^{M}(T), g^{M}(J)\right) \exp \left(-C_{2}\left|g^{S_{r}}(L) \| g^{S_{r}}(R)\right|\right),
\end{gathered}
$$

where the constant $C_{2}$ depends on $g$ and the neighbourhood $T_{0}$, which is fixed. Therefore, by the Standing Assumption and Lemma 4.3, we obtain setting $k(n)=S_{n-1}+$ $\ldots+S_{N}$ :

$$
\begin{gathered}
B\left(g^{k(n)}\left(U_{n-1}\right), g^{k(n)}\left(U_{n}\right)\right) \geq B\left(U_{n-1}, U_{n}\right) \\
\exp \left(-C_{2} \sum_{r=N}^{n}\left|g^{S_{n-1}+\ldots+S_{r+1}}(L)\right|\left|g^{S_{n-1}+\ldots+S_{r+1}}(R)\right|\right) .
\end{gathered}
$$

The latter sum can be made arbitrarily small provided $N$ is large enough, because the collection of intervals:

$$
g^{S_{n-1}+\ldots+S_{r+1}}\left(U_{n}\right) \subset g^{S_{n-1}+\ldots+S_{r+1}}\left(U_{n-1}\right), r=N, \ldots, n,
$$

satisfies the margins disjointness property (because $g^{S_{n-1}+\ldots+S_{r+1}}\left(U_{n}\right)$ lies in the right component of $\left.U_{r} \backslash U_{r+1}\right)$. Then we complete the proof as in Lemma 3.3. Thus Lemma 4 of [Le] and Lemma 3.3 are proved for smooth maps $g$.

Now we can proceed as in [Le] and complete the proof of Proposition 4 of [Le] and the real bounds from Theorem 2.1 for smooth maps as above.

Step 4: The only remaining real estimate we need to prove is Lemma 3.4. But under the condition and notations of the proof of Lemma 3.4, the collection of intervals

$$
g^{j}\left(\hat{U}^{g}\right) \subset g^{j}\left(U^{g}\right), j=0, \ldots, S_{n_{i}}-1,
$$

has the margins disjointness property, because $\hat{U}^{g} \subset U_{n_{i}}^{g}, \partial_{r} U^{g}=\partial U_{n_{i}}^{g}$ and because the intervals $g^{j}\left(U_{n_{i}}^{g}\right), 0 \leq j \leq S_{n_{i}}-1$, are pairwise disjoint. Therefore also the inequality of Lemma 3.4 follows for smooth maps $g$, with a spoiling factor which tends to 1 as $n_{i} \rightarrow \infty$. 


\section{Complex bounds for real analytic maps in the minimal case: the proof of Theorem A}

In this section we prove Theorem A for real analytic maps $g \in A C$. This means that we have to use Lemma 2.3 instead of the Schwarz Lemma (because we only have a conformal map near the dynamical interval). Therefore we shall need that the sum of the lengths of the intervals along any branch of the first return map becomes small (because then we merely have a small loss of angles of pullbacks of the Poincaré domains). To get this, note that the real bounds from the previous section imply that $\omega(c)$ has Lebesguc measure zero (cf. [LS1] and [Ko]).

Let us go through the proofs of the results from Section 3.

First we deal with the real analytic version of Lemma 3.1.

Lemma 5.1 Let $g$ be real analytic as above and assume that there exist infinitely many $n$ 's for which

$$
\max \left(\left|\partial_{l} U_{n}-c\right|,\left|\partial_{r} U_{n}-c\right|\right)<(1+\epsilon) \operatorname{dist}\left(\partial U_{n-1}, c\right)
$$

Then $g$ has complex bounds.

Proof: As before assume that

$$
\left|\partial_{l} U_{n}-c\right| \leq\left|\partial_{r} U_{n}-c\right|<(1+\epsilon)\left|\partial_{l} U_{n-1}-c\right|
$$

Let us distinguish two cases as in Lemma 3.1.

Case I. $U_{n}$ is well-inside $U_{n-1}$. Then, as before, take $\Omega=D_{*}\left(U_{n-1}\right)$. Let $J$ be a domain of the first return map to $U_{n-1}$ which intersects $\omega(c)$ and let $s$ be its return time. We claim that $J$ is woll inside $U_{n-1}$. Indeed, if $J=U_{n}$ then this follows by assumption. So let us assume that $J$ is not the central domain. $g^{s-1}: J^{g} \rightarrow U_{n-1}$ extends to a diffeomorphism $g^{s-1}: \hat{J}^{g} \rightarrow U_{n-2}$ with $\hat{J} \subset U_{n-1}$. So if $U_{n-1}$ is well inside $U_{n-2}$ then $J$ is also well inside $U_{n-1}$. If $U_{n-1}$ is not well inside $U_{n-2}$, then $U_{n}$ is part of a long chain $U_{n+i} \subset \ldots \subset U_{n-k}$. But then, since $J \neq U_{n}$, we get that $g^{s-1}: J^{g} \rightarrow U_{n-1}$ extends to a diffeomorphism $g^{s-1}: \hat{J}^{g} \rightarrow U_{n-k-1}$ with $\hat{J} \subset U_{n-1}$. By the real bounds $U_{n-1}$ is well inside $U_{n-k-1}$, and so $J$ is well inside $\hat{J} \subset U_{n-1}$. Therefore the claim is proved in all situations. Since $|\omega(c)|=0$, the sum of the lengths of $J, \ldots, f^{s-1}(J)$ is small when $n$ is large. It follows from Lemma 2.3 that the pullback of $\Omega=D_{*}\left(U_{n-1}\right)$ to $J$ (with $J$ either a non-central domain or equal to $U_{n}^{g}$ ) is contained in a Poincaré disc based on $J$ with angle close to $\pi / 2$ when $n$ is large. Since $U_{n}$ is well inside $U_{n-1}$ the central domain is still contained well inside the range $\Omega$ (using the same arguments as in Case 1 of Lemma 3.1). By the claim above, a non-central domain $J$ is well inside $U_{n-1}$, and so the Poincaré disc based on $J$ with angle close to $\pi / 2$ is again contained well inside $\Omega$.

Case II. The interval $U_{n}$ is not well inside $U_{n-1}$. Then $U_{n}$ is inside a cascade of central domains. As in Lemma 3.1 define $m \geq n \geq n-k-1$ so that $S_{m+1}>S:=$ $S_{m}=\ldots=S_{n-k}>S_{n-k-1}$. As in Lemma 3.1 let $U_{*}$ be the smallest symmetric interval containing $U_{m}$. As before, $U_{*}$ is well inside $U_{n-k-1}$. Let $\Omega=D_{*}\left(U_{*}\right) \backslash\left(U_{*} \backslash U_{m}\right)$ and 
let $U$ with $U_{m} \supset U \supset U_{m+1}$ be so that $g^{S_{m+1}-1}: U \rightarrow U_{*}$ is a homeomorphism. Since $|\omega(c)|=0$ and the intervals $g^{j}\left(U_{m+1}\right), j=0, \ldots, S_{m+1}-1$ are pairwise disjoint, the sum of the lengths of these intervals is arbitrary close to zero, when $n$ (and therefore $m$ ) is large enough. Since $U_{*}$ is well inside $U_{n-k-1}$ and has length $\leq 2\left|U_{m}\right|=\left|2 \cdot g^{S_{m+1}}\left(U_{m+1}\right)\right|$, and since $g^{S_{m+1-1}}: U^{g} \rightarrow U_{*}$ extends to a diffeomorphism onto $U_{n-k-1}$, we get by the Koebe Principle that the sum of the lengths of $g^{j}(U), j=0, \ldots, S_{m+1}-1$, is close to zero when $n$ and $m$ are large. Therefore, the preimage of the disc $D_{*}\left(U_{*}\right)$ by the central branch to the critical value $c_{1}$ is contained in a set $D\left(U^{g}, \theta\right)$, where the angle $\theta$ is close to $\pi / 2$ for $n$ large. Since $U$ is well inside $U_{m} \subset U_{*}$, we get that the central domain is contained well inside $\Omega$. Let us now consider a non-central domain $J$ of the return map to $U_{m}$ with return time $s$. Let $\hat{J} \supset J$ be so that $g^{s}: \hat{J} \rightarrow U_{*}$ is a homeomorphism. The problem is that the pullback of a Euclidean disc is merely inside a Poincaré disc of angle close to $\pi / 2$. Therefore we need that $\hat{J}$ is well inside $U_{*}$. This holds for all noncentral domains with return time $s>S_{m}$, and so for these the corresponding donains in the complex plane fit inside $\Omega$. However, unfortunately, for the non-central branch $g^{S_{m}}: I_{m} \rightarrow U_{m}$ this may not hold. Indeed, let $\hat{I}_{m} \supset I_{m}$ be so that $g^{S_{m}}: \hat{I}_{m} \rightarrow U_{*}$ is a homeomorphism. It need not be true that $\hat{I}_{m}$ is well inside $U_{*}$. Therefore, let us change the definition of $U_{*}$ slightly. Define $U_{*}(\tau)$ to be the interval with boundary points $\pm(1+\tau) \partial_{r} U_{*}$. For all $\tau \in\left[0, \tau_{0}\right]$ (with $\tau_{0} \in(0,1)$ some fixed small number), the interval $U_{*}(\tau)$ is still well inside $U_{n-k-1}$ and there is an interval $\hat{I}_{m}(\tau) \ni c$ such that $g^{S-1}: \hat{I}_{m}(\tau)^{g} \rightarrow U_{*}(\tau)$ is a diffeomorphism. Since $g$ has no periodic attractors or neutral periodic orbits near $c$ (see see Proposition 2.1), we may assume that $n$ is so large that $g^{S}: \hat{I}_{m}(\tau) \rightarrow U_{*}(\tau)$ has only one fixed point. This fixed point is repelling and lies in $U_{m}$.

Claim: There exists $\tau \in\left[0, \tau_{0}\right]$ so that $\hat{I}_{m}(\tau)$ is well inside $U_{*}(\tau)$. To prove this claim, consider $\tau_{i}=(i / 8) \tau_{0}$ with $i=1, \ldots, 8$ and let $\left[l_{i}, r_{i}\right]=\hat{I}_{m}\left(\tau_{i}\right)$. If the claim is falise for $\tau_{i}$ then either

$$
\left|g^{S}\left(r_{i}\right)-r_{i}\right| \leq o(n)\left|U_{*}\right| \text { or }\left|g^{S}\left(l_{i}\right)-l_{i}\right| \leq o(n)\left|U_{*}\right|
$$

where $o(n)$ are functions which tend to zero as $n \rightarrow \infty$. So if the claim is false for all $i$, then for at least four of the points $l_{1}, \ldots, l_{8}$ we have

$$
\left|g^{S}\left(l_{i}\right)-l_{i}\right| \leq o(n)\left|U_{*}\right| \text { as } n \rightarrow \infty
$$

(or the same holds for four of the points $r_{i}$ ). Choose intervals $T \supset J$ so that $C(T, J)$ is the cross-ratio determined by these four points (say from $l_{\mathbf{1}}, \ldots, l_{8}$ ). From (5.1) it follows that $C\left(g^{S}(T), g^{S}(J)\right) / C(T, J)$ is close to one when $n$ is large. But since $U_{*}(\tau)$ is well-inside $U_{n-k-1}$ we get from the (real) Koebe principle that the non-linearity of $g^{S-1}: \hat{I}_{m}\left(\tau_{0}\right) \rightarrow U_{*}\left(\tau_{0}\right)$ is universally bounded. Therefore and by the definition of $\tau_{i},|J|$, $|l(T \backslash J)|,|r(T \backslash J)|$ and $\left|U_{*}\right|$ are all of the same order. But an explicit calculation for the map $z \mapsto z^{\ell}$ shows that this implies that $C(g T, g J) / C(T, J)>1+\kappa$ for some universal constant $\kappa>0$ (the intervals $J \subset T$ do not contain the critical point $c=0$, and are not small compared to the distance to $c)$. Since $\hat{I}_{m}\left(\tau_{0}\right) \subset U_{n-k}$ and the total length of the intervals $U_{n-k}, \ldots, f^{S-1} U_{n-k}$ tends to zero (since $\left.|\omega(c)|=0\right), \frac{C\left(g^{S} T, g^{S} J\right)}{C(g T, g J)} \geq 1-o(n)$ 
where $o(n) \rightarrow 0$ as $n \rightarrow \infty$. It follows that

$$
\frac{C\left(g^{S} T, g^{S} J\right)}{C(T, J)} \geq 1+\kappa-o(n) .
$$

But this violates (5.1). This contradiction proves the claim.

If we now define

$$
\Omega(\tau)=D_{*}\left(U_{*}(\tau)\right) \backslash\left(U_{*}(\tau) \backslash U_{m}\right)
$$

then the extension of all non-central domains are all contained well irside $U_{*}(\tau)$. Thus we obtain a quasi-polynomial-like map.

Next we should remark that Lemmas $3.2,3.3,3.4$ and 3.5 remain unchanged or were proved already in the previous section. So we only need to prove lemma 3.6. To do this, simply proceed as in the proof of Lemma 5.1 (notice that in Case II we have $\left.|V| \leq 3\left|U_{n}\right|\right)$. This completes the proof of Theorem A (complex bounds).

\section{Polynomial-like structure of real analytic maps in the minimal case}

Given a real analytic map $g \in \mathbf{A C}$ with $\omega(c)$ minimal, we have constructed a sequence of quasi-polynomial-like mappings

$$
P_{k}: \cup_{i} V_{k}^{i} \rightarrow V_{k}
$$

such that $\operatorname{diam}\left(V_{k}\right) \rightarrow 0$, the map $P_{k}$ is the analytic extension from the real line of a first return map $g$ to an interval $U_{n_{k}}$ of our sequence (so that the intersection of $V_{k}$ with the real axis is $U_{n_{k}}$ ). Quasi-polynomial-like mappings have domains which can intersect.

In this section we shall show how to construct from this polynomial-like mappings

$$
P_{k}: \cup_{i} \hat{V}_{k}^{i} \rightarrow \hat{V}_{k}
$$

Here $\hat{V}_{k}^{i}, \hat{V}_{k}$ will be subsets of $V_{k}^{i}, V_{k}$, and $P_{k}$ are restrictions of the quasi-polynomial-like mappings $P_{k}: \cup_{i} V_{k}^{i} \rightarrow V_{k}$.

We should emphasize that the polynomial-like maps $P_{k}: \cup_{i} \hat{V}_{k}^{i} \rightarrow \hat{V}_{k}$ we will build here may have lost the original property of the quasi-polynomial-like maps $P_{k}: \cup_{i} V_{k}^{i} \rightarrow$ $V_{k}$ that the moduli of annuli between the ranges and the central domains are uniformly bounded away from zero. In spite of this, we can conjugate the map $P_{i}: \cup_{i} \hat{V}_{k}^{i} \rightarrow \hat{V}_{k}$ to a polynomial, see the next Section.

The construction will go in two steps.

Step 1: Construction of a smooth polynomial-like mapping. Given the initial map $g: \cup_{i} I_{i} \rightarrow I$, one can assume that the closures of the intervals $I_{i}$ are pairwise disjoint and contained in the open interval $I$ (we can always pass to a first 
return map along the postcritical set, which, by the assumption that $w(c)$ is minimal, obeys such property). Let us choose a neighbourhood $V$ of the interval $I$ (with $V$ a topological disc with analytic boundary) so close to $I$, that, for cach $i=0, \ldots, i_{0}$, there exists a neighbourhood $U_{i}$ of the interval $I_{i}$ such that for $i \neq 0$ the map $: U_{i} \rightarrow V$ is a complex analytic (diffeomorphic) covering map. Choose $V$ so that the cises $U_{i}$ are disjoint. For $i=0$ it might be impossible to extend g| I0 antalytically to some set $U_{0}$ in such that $g: U_{0} \rightarrow V$ becomes an analytic branched (civering map). (If sich an extension did exist, its preimage of $V$ would not be close to the real line.) So instead choose an analytic extension near $I_{0}$ and then extend diffeomotphically so that $g: U_{0} \rightarrow V$ becomes a smooth branched covering map with critical point. $c$. Note that $U_{i}$ are not necessarily contained in $V$, but we can make sure that all the topological $\operatorname{discs} U_{i}$ are disjoint. Therefore, fix a topological disc $A$, which properly contains $V$ and each $U_{i}$. Given $i$, choose a disc $B_{i}$, which contains properly $U_{i}$, is contained properly in $A$, and so that all $B_{i}$ are pairwise disjoint. Let us artificially extend $g: U_{i} \rightarrow V$ to a map $\tilde{g}: B_{i} \rightarrow A$, which is a diffeomorphism for $i \neq 0$ and such that it is a covering map for $i=0$. After this step, we obtain

$$
\tilde{g}: \cup_{i} B_{i} \rightarrow A,
$$

which is an extension of $g \cup_{i} U_{i} \rightarrow V$, such that each $\tilde{g}: B_{i} \rightarrow A$ is a diffeomorphisn for $i \neq 0$, and $\tilde{g}: B_{0} \rightarrow A$ is a covering map with a unique critical point at $c$. This map is the intermediate smooth polynomial-like mapping. Note that there is NO guarantee that $\tilde{g}$ and $g$ coincide wherever they are both defined and that $\tilde{g}$ is not analytic. However, $g=\tilde{g}$ close to the intervals $I_{i}$.

Step 2: Intersecting the quasi-polynomial-like mapping with the smooth polynomial-like mapping. Consider the sequence of first return maps of $\tilde{g}$ along the postcritical set. Thus we obtain a sequence of smooth polynomial-like mappings

$$
\tilde{g}_{n}: \cup_{i} B_{i}^{n} \rightarrow A^{n}
$$

such that $\tilde{g}_{n}$ coincides with the first return map of $g$ to $U_{n}$ on the real traces. This means that the smooth polynomial-like map $\tilde{g}_{n_{k}}$ coincides with the quasi-polynomiallike map $P_{k}$ on the real line. We remark that one of the properties of the grasipolynomial-like mapping $P_{k}$ is that if $V_{k}^{i}$ is one of its domains with $P_{k} \mid V_{k}^{i}=g^{s(i, k)}$ then $V_{k}^{i}, \ldots, g^{s(i, k)}\left(V_{k}^{i}\right)$ are all contained in small Poincaré neighbourhoods of the real trace of these sets. So take the component of $A^{n_{k}} \cap V_{k}$. which contains $c$ and consider the corresponding preimages by $P_{k}$ and $\tilde{g}_{n_{k}}$. By the above remark, $\dot{g}_{n_{k}}$ coincides with $P_{k}$. on these preimages. Thus we get a truly polynomial-like mapping,

$$
P_{k}: \cup_{i} \hat{V}_{k}^{i} \rightarrow \hat{V}_{k},
$$

see also [LS1].

Note that the constructed polynomial-like maps $P_{k}: \cup_{i} \hat{V}_{k}^{i} \rightarrow \hat{V}_{k}$ possibly lost the property of the quasi-polynomial-like maps $P_{k}: \cup_{i} V_{k}^{i} \rightarrow V_{k}$, that the moduli of anmuli between the ranges and the central domains are uniformly away from zero, but on the 
other hand we can (and will) conjugate the map $P_{k}: \cup_{i} \hat{V}_{k}^{i} \rightarrow \hat{V}_{k}$ with a polynomial, see next Section.

Take one of the constructed polynomial-like maps $P_{k}: \cup_{i} \hat{V}_{k}^{i} \rightarrow \hat{V}_{k}$. By Theorem A, it induces the quasi-polynomial-like maps $P_{m}: \cup_{i} V_{m}^{i} \rightarrow V_{m}$ for $m>>k$ sufficiently large (with definite bounds for the moduli of the annuli obtained by taking the difference of the range and the central domain). But as in [LS2] this is enough to obtain

Theorem 6.1 1. Every polynomial-like map $P_{k}: \cup_{i} \hat{V}_{k}^{i} \rightarrow \hat{V}_{k}$ has totally disconnected Julia set.

2. It carries no invariant line field on its Julia set.

\section{Conjugation to polynomials in the minimal case}

Let us fix a polynomial-like map $P: \cup_{i} \hat{V}^{i} \rightarrow \hat{V}$ from the sequence of such maps just constructed, but without any restriction on the postcritical set of the non-escaping critical point 0 except that we still require that all iterates of the critical point stay in the domain of definition of $P$ (for example, $P$ can have an attracting periodic orbit). All domains considered in this section will be simply connected and symmetric with respect to the real axis. We also write $U<V$, if two domains $U, V$ like this have disjoint closures and such that the intersections of $U$ and $V$ with the real axis are ordered accordingly. For example, we can order the domains $\hat{V}^{i}$ like this. Let us relabel the domains so that in this new notation the map is:

$$
P: U_{-2 m^{-}} \cup U_{-2\left(m^{-}-1\right)} \cup \ldots \cup U_{0} \cup \ldots \cup U_{2\left(m^{+}-1\right)} \cup U_{2 m^{+}} \rightarrow V,
$$

where $m^{ \pm}$the number of domains to the left and right of the central domain $U_{0}$ (which contains the critical point $c$ ), and such that

$$
U_{-2 m^{-}}<U_{-2\left(m^{-}-1\right)}<\ldots<U_{0}<\ldots<U_{2\left(m^{+}-1\right)}<U_{2 m^{+}} .
$$
so that

Let us add new domains $U_{-\left(2 m^{-}+1\right)}, U_{-\left(2 m^{-}-1\right)}, \ldots, U_{-1}, \ldots, U_{2 m^{+}-1}, U_{2 m^{+}+1}$ ordered $U_{-\left(2 m^{-}+1\right)}<U_{-\left(2 m^{-}\right)}<U_{-\left(2 m^{-}-1\right)}<\ldots<U_{-1}<U_{0}<\ldots<U_{2 m^{+}-1}<U_{2 m^{+}}<U_{2 m^{+}+1}$.

For each new domain, we choose a holomorphic isomorphism, which maps this domain onto $V$, such that this map is real and reverses orientation on the real axis.

We obtain a new polynomial-like map

$$
Q: \cup_{i=-\left(2 m^{-}+1\right)}^{2 m^{+}+1} U_{i} \rightarrow V
$$

with a unique critical point. This critical point is $c=0$ and is of odd order $\ell \geq 3$.

Theorem 7.1 $Q$ is quasiconformally conjugate to a polynomial $F$, in neighbourhoods of their filled-in Julia sets, where $F$ is such that: 
- The degree of $F$ is equal to $d=\ell+2\left(m^{-}+1\right)+2\left(m^{+}+1\right)$, and it is of the form $F(z)=z^{\ell} \cdot r(z)+C$, where $r(z)=z^{2\left(m^{-}+m^{+}+2\right)}+\ldots$ is a monic polynomial with real coefficients, and $C=F(0)$ is a real number.

- All critical points of $F$,

$$
x_{-\left(2 m^{-}+1\right)}<x_{2 m^{-}}<\ldots<x_{-1}<0<x_{1}<\ldots<x_{2 m^{+}+1}
$$

are real (and can be ordered like this), and such that

$$
F\left(x_{i}\right)=H_{-} \text {and } F\left(x_{j}\right)=H_{+}
$$

for $i$ odd and $j \neq 0$ even with $-\left(2 m^{-}+1\right) \leq i, j \leq 2 m^{+}+1$ where $H_{ \pm}$are real.

- All critical points of $F$ except 0 escape to infinity, and, if we denote by $B$ the Bottcher function of the basin of infinity of $F$ (normalized so that $B(z) / z \rightarrow 1$ as $z \rightarrow \infty)$, then $B\left(H_{-}\right)=-2$ and $B\left(H_{+}\right)=2$.

Proof: We can assume that the boundaries of the domains $U_{i}, V$ are analytic curves (taking a little bit smaller disc inside $V$ if necessary). By applying a conjugacy which maps $V$ conformally onto a round disc $D_{R}=\{|z|<R\}$, for some $R>1$, such that $R<2<R^{d}$, we can and will assume that $V=D_{R}$. Our map $Q$ is defined so far on the union of $U_{i}$. Define also

$$
Q(z):=z^{d}
$$

off $V$. Therefore $Q(V)=D_{R^{d}}$. Now choose a map

$$
\phi: V \backslash \cup_{i} \bar{U}_{i} \rightarrow Q(V) \backslash \bar{V}
$$

such that:

1. $\phi$ is a smooth $d$-fold branched covering map (not holomorphic) of the domain $V \backslash \cup_{i} \bar{U}_{i}$ onto the standard annulus $Q(V) \backslash \bar{V}$, which extends smoothly onto the boundaries, such that it agrees with $Q$. That is, $\phi=Q$ on $\partial\left(V \backslash \cup_{i} \bar{U}_{i}\right)$.

2. $\phi$ is real on the real axis, and, moreover, $\phi(\bar{z})=\overline{\phi(z)}$. All critical points of the cover $\phi$ are real (this is possible because the orientations of $Q$ on the real traces of any two adjacent domains $U_{i}, U_{i+1}$ are opposite and because $\phi=Q$ on $\partial L_{0}^{\prime}$ ).

3. Denote the critical points of $\phi$ by $a_{-.\left(2 m^{-}+1\right)}<a_{2 m^{-}}<\ldots a_{-1}<a_{0}<a_{1}<\ldots<$ $a_{2 m^{+}}$, where $a_{i}$ lies between $U_{i}$ and $U_{i+1}$. Then

$$
\phi\left(a_{i}\right)=-2, \quad \phi\left(a_{j}\right)=2
$$

for $i$ odd and $j$ even with $-\left(2 m^{-}+1\right) \leq i, j \leq 2 m^{+}+1$.

4. $\phi$ is a local diffeomorphism at any point $\neq a_{i}$; near each critical point $a_{i}$ the map $\phi$ is holomorphic (and non-singular). 
Now we define $Q(z)=\phi(z)$, for every $z \in V \backslash \cup_{i} \bar{U}_{i}$. Thus $Q$ is defined everywhere.

We are in a position to use the well-known trick from the Straightening Theorem [DH]. Take a standard conformal structure (i.e., the Beltrami coefficient $\mu=0$ ) outside the disc $Q(V)$ and extend this structure to a function $\mu: \mathbb{C} \rightarrow\{z ;|z|<1\}$ which is invariant under $Q$. Since $Q$ is conformal off $V$ and on $\cup U^{i}$, there are only a bounded number of points (namely, one) in each orbit of $Q$ where this map is not conformal. It follows that the $L^{\infty}$-norm of $\mu(z)$ is bounded away from one, and by the Measurable Riemann Mapping Theorem, there exists a quasiconformal homeomorphism $H: \overline{\mathbb{C}} \rightarrow \overline{\mathbb{C}}$ with $H(\infty)=\infty$ which has $\mu$ as its Beltrami coefficient. Because the structure is symmetric with respect to the real line, $H(\bar{z})=\overline{H(z)}$. Since $\mu$ is invariant under $Q$, it follows that

$$
F=H \circ Q \circ H^{-1}
$$

is a holomorphic $d$-cover of the complex plane. Hence $Q$ is quasiconformally conjugate to a polynomial map $F$ (of degree $d$ ). By an affine conjugacy, we can assume that $F$ is monic (it means that $H(z) / z \rightarrow 1$ at $\infty$ ), and $H(c)=0$. Then $x_{i}=H\left(a_{i}\right)$, $i=-\left(2 m^{-}+1\right), \ldots, 2 m^{+}$are all simple real critical points of $F$. Together with $0=h(c)$ these are the only critical points of $F$. The critical point $0=H(c)$ is of order $\ell$. By construction,

$$
F\left(x_{2 k+1}\right)=H(-2):=H_{-}, \quad F\left(x_{2 k}\right)=H(2):=H_{+} .
$$

$H$ conjugates $Q(z)=z^{d}$ to $F$ in the domain $\mathbb{C} \backslash V=\{|z|>R\}$, and is holomorphic there (because $H$ preserves the standard complex structure off $V$ ). Moreover, $H(z) / z \rightarrow 1$ at $\infty$. Hence, the inverse map $B=H^{-1}$ coincides with the Bottcher function. That is, $B\left(H_{-}\right)=-2, B\left(H_{+}\right)=2$.

Complement to Theorem 7.1: the polynomial $F$ can be chosen to depend continuously on the polynomial-like map $P$ provided the polynomial-like map $P$ carries no invariant line fields on its filled-in Julia set $K(P)$. (In particular, assume that $P$ has no periodic attractor.) (Here convergence is meant in the Caratheodory topology: convergence of the domains to a kernel, and convergence of holomorphic maps uniformly on compacta, see e.g. [McM1]). Let us first observe that this implies that the polynomial $F$ also carries no invariant line field on its filled-in Julia set $K(F)$. Indeed, the conjugacy $H$ has to be conformal a.e. on $K(P)$ because otherwise the preimage of the standard complex structure on $K(F)$ by $H$ gives an invariant non-standard one on $K(P)$, that is, an invariant line field. Hence $H$ is conformal on $K(P)$, and so it takes an invariant line field of $P$ to an invariant line field of $F$, and vise versa. This proves the observation. Now, let $P_{n} \rightarrow P$ be a sequence of polynomial-like maps tending to $P$. Then we can choose $Q_{n}: \cup_{i} U_{i}^{n} \rightarrow V^{n}$ converging to $Q: \cup_{i} U_{i} \rightarrow V$ uniformly on compact subsets of the complex plane and so that the Beltrami coefficient of $Q_{n}$ on $V^{n} \backslash \cup_{i} U_{i}^{n}$ converges to the one of $Q$ on $V \backslash \cup_{i} U_{i}$ in $L^{\infty}$-norm (even in sup-norm). If $H_{n}$ is the quasiconformal homeomorphism corresponding to $Q_{n}$, and $F_{n}=H_{n} \circ Q_{n} \circ H_{n}^{-1}$ is the corresponding polynomial, then the norms of the Beltrami coefficients of $H_{n}$ are uniformly away from 1 . Since all $H_{n}$ are normalized in the same way, passing to a subsequence, $H_{n} \rightarrow H_{*}$, where $H_{*}$ is quasiconformal and conformal at $\infty$. We get 
that any such subsequence $F_{n}$ tends to a polynomial $F_{*}=H_{*} \circ Q \circ H_{*}^{-1}$ and that $H^{-1} \circ F \circ H=H_{*}^{-1} \circ F_{*} \circ H_{*}$. But $F, F_{*}$ are monic polynomials of the same degree and $H, H_{*}$ are homeomorphisms of the plane which are conformal at $\infty$. Then, by the last equality, the quasiconformal homeomorphism of the plane $H \circ H_{*}^{-1}$ (which coniugates $F$ and $F_{*}$ ) is conformal off the filled-in Julia set of the polynomial $F_{*}$. If the quasiconformal homeomorphism $H \circ H_{*}^{-1}$ would not be not conformal a.e. on the filled-in Julia set of the polynomial $F$, then the complex structure, which is the image of the standard one by the map $H \circ H_{*}^{-1}$, is invariant under $F$. Hence, $F$ carries an invariant line field on its filled-in Julia set, a contradiction. Therefore, $H \circ H_{*}^{-1}$ is conformal a.e.. So this map is affine and by the normalization it is equal to the identity map. Hence, from Theorem 6.1,

Corollary 7.1 If $P$ is a polynomial-like map constructed in the previous section with $\omega(c)$ minimal, and if $P_{n} \rightarrow P$ is a sequence of polynomial-like maps, then $F_{n} \rightarrow$ $F$ (where $F_{n}, F$ are the corresponding polynomials from the previous theorem). (The convergence in this lemma is in the Caratheodory topology).

Proof: In the previous section we constructed polynomial-like maps $P$ from maps $g$ with $\omega(c)$ minimal, and showed that these do not carry invariant linefields on their filled Julia set.

\section{Conjugations to expanding maps of a circle}

Let $g: \cup_{i=0}^{i_{0}} I_{i} \rightarrow I$ be a real analytic map of the class we consider, but without any restriction on the postcritical set of the critical point $c$. Let us only assume that all iterates of the critical point stay in the domain of definition of $g$ (for example, $g$ can have an attracting periodic orbit). For simplicity also assume that each $I_{i}$ is compactly contained in the interior of $I$. (This is the case for all first return maps, if we assume that $\omega(c)$ is minimal).

Let us assume for simplicity that the length of $I$ is equal to 1 . Then we can find an orientation preserving homeomorphism $G: \mathbb{R} \rightarrow \mathbb{R}$ so that $G(x+1)=G(x)+1$ for all $x$ and so that $G \mid \cup I_{i}=g \bmod 1$. We can do this so that each component $J$ of $I \backslash \cup I_{i}$ is mapped by $G$ affinely onto an interval of length 1 . Note that $G$ induces a piecewise smooth covering map $\hat{g}: S^{1} \rightarrow S^{1}$. Denote by $m$ the number of branches of the map $\hat{g}$.

Let $G_{m}(z)=m \cdot z \bmod 1$ be the affine $m$-covering of the circle $S^{1}=\mathbb{R} \bmod 1$. It is well known (see e.g. [MS]), that there exists an order preserving and surjective map $h: S^{1} \rightarrow S^{1}$, such that $h \circ \hat{g}=G_{m} \circ h$. This map $h$ is unique if we insist that $h$ maps the fixed point corresponding to $\partial I \bmod 1$ to $0 \bmod 1$. One can define $h$ by letting $h(x)$ to be the point whose kneading invariant with respect to $G_{m}$ is the same as the kneading invariant of that of $x$ with respect to $\hat{g}$. (For example, the kneading invariant of $x$ w.r.t. $\hat{g}$ lists for all $n$ which component of $S^{1} \backslash g^{-1}(0)$ the point $f^{n}(x)$ hits.) Uniqueness of $h$ follows since the choice $h(\partial I \bmod 1)=0 \bmod 1$ fixes the way we label these components.) Of course, $h$ will be constant on each component of a basin of a periodic attractor of $\hat{g}$. 
It is clear that $h$ depends continuously on $g$. Indeed, if $\bar{g}$ is a maf from our class which is close to $g$ (in the $C^{0}$-topology), then $h_{\bar{g}}$ is close to $h_{g}$ (in $C^{0}$-topology). In particular, the image $h_{g}(c) \in S^{1}$ of the critical point $c$ of $g$ depends continuously on $g$.

For any real $\epsilon$ with $|\epsilon|$ small. consider a perturbation $g_{\epsilon}: \cup_{i=0}^{i_{0}} I_{i} \rightarrow I^{\epsilon}$, where $g_{\epsilon}(x)=g(x)+\epsilon$, and $I^{\epsilon}=I+\epsilon$. Let $G_{\epsilon}=G+\epsilon$, and $\hat{g}_{\epsilon}$ be the the corresponding covering map of $S^{1}$. Then $\hat{g}_{\epsilon}$ has a fixed point $p_{\epsilon}$ depending continuously on $\epsilon$. Without loss of generality we may assume that $I=[0,1]$ so that $p_{0}=0$. By insisting that $h_{g_{\epsilon}}\left(p_{\epsilon}\right)=0$ (i.e. taking kneading invariants with respect to the partition $S^{1} \backslash \hat{g}_{\epsilon}^{-1}\left(p_{\epsilon}\right)$ which depends continuously on $\epsilon$ when $\epsilon$ is close to zero), we have a continuous function

$$
\alpha(\epsilon)=h_{g \epsilon}\left(c^{\circ}\right.
$$

from a closed interval $\left[-\epsilon_{0}, \epsilon_{0}\right]$ into the unit circle $S^{1}$.

Lemma 8.1 The function $\epsilon \mapsto \alpha(\epsilon)$ is order preserving for $\epsilon$ close to zero. If the critical point of $g$ is not in the closure of a component of the basin of a periodic attractor, then $\alpha\left(\left[-\epsilon_{0}, \epsilon_{0}\right]\right)$. contains an open arc of $S^{1}$.

Proof: Take first $\epsilon>0$ and note that

$$
G_{\epsilon}^{n}(x) \geq G^{n}(x)+\epsilon \text { for all } x .
$$

Consider the partition generated by $g_{\epsilon}^{-n}\left(p_{\epsilon}\right), n \geq 1$. Note that $p_{\epsilon}<p_{\mathrm{J}}=0$ for $\epsilon>0$ small (just look at the graphs of $g_{\mathrm{e}}$ for different choices of $\epsilon$ ). Fix $n>0$ and let $x_{n}$ be a solution of $g^{n}\left(x_{n}\right)=0$. Then there exists $x_{n, \epsilon}$ depending continuously on $\epsilon>0$ (when $\epsilon>0$ is small) so that $g_{\epsilon}^{n}\left(x_{n, \epsilon}\right)=p_{\epsilon}$. We have

$$
x_{n, \epsilon}<x_{n}
$$

because otherwise (since $G_{\epsilon}$ is increasing and by (8.1)) $G_{t}^{n}\left(x_{n, \epsilon}\right) \geq G_{\epsilon}^{n}\left(x_{n}\right)>G^{n}\left(x_{n}\right)+$ $\epsilon=p_{0}+\epsilon>0>p_{\epsilon}$, a contradiction. This and (8.1) imply that

$$
c \mapsto \alpha(c)=h_{y_{e}}(c)
$$

is non-decreasing. Since $g_{\mathrm{f}}$ has no wandering intervals and $c$ is not contained in the closure of a component of the basin of a periodic attractor of $\hat{g}$, elements of the partition containing $g(c)$ shrink to zero in size. This, (8.1) and (8.2) imply that the kneading coordinates of $c$ with respect to $g$ and $g_{\epsilon}$ are not the same, i.e. $\alpha(\epsilon)>\alpha(0)$. Considering also negative $\epsilon$, together with the continuity of $\alpha$, the lemma follows.

\section{Quasiconformal rigidity in the minimal case: proof of Theorem B}

Theorem 9.1 Let $g, g_{1}$ be two real analytic maps from our class, such that the $\omega$-limit sets of the critical points of the maps are minimal. Assume that $g, g_{1}$ are topologically 
conjugate by a homeomorphism, which maps the critical point of $g$ to the critical point of $g_{1}$. Then some maps $G=g^{S_{n}}$ and $G_{1}=g_{1}^{S_{n}}$ from the sequences of first return maps of $g$ and $g_{1}$ extend analytically to polynomial-like maps. These two polynomial-like maps are quasiconformally conjugate in neighbourhoods of their Julia sets by a map which is conformal on their Julia sets.

Proof: Provided that the quasiconformal conjugacy does exists, the latter statement (conformality of the conjugacy) follows from the fact that the polynomial-like maps induced by $g$ and $g_{1}$ have no invariant line ficlds on their filled-in Julia sets. Let us prove the existence of the (quasiconformal conjugacy. We can assume that the critical points of $g$ and $g_{1}$ are at zero. As we have proved, there exists a polynomial-like extension $P$ of some first return $g^{S_{n_{1}}}$ of $g$, and a polynomial-like extension $P_{1}$ cf some first return $g_{1}^{S_{n_{2}}}$ of $g_{1}$. If, say, $S_{n_{2}}>S_{n_{1}}$, then, because the maps $g, g_{1}$ are corjugate and the conjugacy respects the critical orbits, we can take first returns to the central domains passing from $P$ to a polynomial-like extension of $g^{S_{n_{2}}}$. Thus, from the beginning, we can assume that $P, P_{1}$ are polynomial-like extensions of some $g^{S_{n}}$ and $g_{1}^{S_{n}}$, such that $P, P_{1}$ are topologically conjugate on the real line. Let $F$ and $F^{1}$ be polynomials which are quasiconformally conjugate to $P$ respectively $P_{1}$ (see Theorem 7.1). To prove the statement, it is enough to show that $F=F^{1}$. Define $P_{\epsilon}(z)=P(z)+\epsilon$ and $P_{\epsilon}^{1}(z)=P_{1}(z)+\epsilon$, with $\epsilon$ real and $|\epsilon|$ small. According to Lemma 8.1, there exist a sequence $\epsilon_{n} \rightarrow 0$ and a sequence $\epsilon_{n}^{\prime} \rightarrow 0$, such that the orbits of the critical point 0 by the map $P_{\epsilon_{n}}$ and by the map $P_{\epsilon_{n}^{\prime}}^{1}$ are well defined, have the same itineraries, and are pre-periodic (but not periodic). In particular, the iterates of the critical point of these maps pass through the same domains as that of $P$. Let $q_{n}, q_{n}^{1}$ be polynomials corresponding to $P_{\epsilon_{n}}$ and $P_{\epsilon_{n}^{\prime}}^{1}$ respectively. By Corollary 7.1, $q_{n} \rightarrow F$ and $q_{n}^{1} \rightarrow F^{1}$ as $n \rightarrow \infty$. Let us for the moment fix $q=q_{n}$ and $q^{1}=q_{n}^{1}$. Then $q, q^{1}$ have the same degree $d$. The non-escaping critical point 0 of $q$ and $q^{1}$ is preperiodic with the same itineraries with respect to the partitions on the real line given by $q$ and $q^{1}$. Since the only non-escaping critical point of $q$ (and $q^{1}$ ) is preperiodic and not periodic, the Julia set of $q$ (and $q^{1}$ ) is totally disconnected [BH]. Denote by $B$ and $B^{1}$ the Böttcher functions at infinity for $q$ and $q^{1}$ respively. Then, by the construction of the polynomials $q, q^{1}$, the map $H=\left(B^{1}\right)^{-1} \circ B$ is conformal in the domain $\{z: \log |B(z)|>(\log 2) / d\}$. Since the itineraries coincide we get, step by step, that the conjugacy $H$ extends conformally onto domains $\left\{z: \log |B(z)|>(\log 2) / d^{n}\right\}, n=1,2, \ldots$. Hence it extends up to the Julia set $J(q)$ of $q$, and then homeomorphically to the Julia set $J(q)$ (because the Julia sets are totally disconnected). It follows that there exists a quasiconformal homeomorphism $h$ of the plane, which is homotopic to $H$ and coincides with $H$ in the domain $\left\{z: \log |B(z)|>(\log 2) / d^{m}\right\}$, for some sufficiently large (but fixed) $m$, and on the postcritical set of $q$ (which is finite). Then, by the pullback argument, there exists a quasiconformal conjugacy between $q$ and $q^{1}$ which coincides with $H$ outside $J(q)$ (and hence is conformal outside $J(q)$ ). Since the extension to $J(q)$ is unique, all this implies that $H$ is quasiconformal. On the other hand, $J(q)$ has Lebesgue measure zero ( $q$ is geometrically finite). Therefore, $H$ is conformal everywhere, i.e., affine. Because of the normalization at infinity, $H$ is the identity. This means that $q=q^{1}$. Remembering that $q$ and $q^{1}$ can be chosen arbitrary close to $F$ and $F^{1}$ respectively, we get that $F=F^{1}$. 


\section{Theorem A': Complex bounds in the non-minimal case}

Let $a_{ \pm}$be the right and left most fixed points of $g$. In addition, let $a_{i}$ be the boundary points of immediate basins of periodic attractors. Because $g$ is real aralytic, $g$ has only finitely many periodic attractors (see [MS]), and there are only finitely many such points $a_{1}, \ldots, a_{s}$. Every point $a_{ \pm}, a_{1}, \ldots, a_{s}$ is either repelling or parabolic periodic. Now define $A=\left\{a_{-}, a_{+}, a_{1}, \ldots, a_{s}\right\} \cap \omega(c)$ and if this is empty let $A$ be any repelling periodic orbit of $g$. (One could also use different partitions, see for example the construction described in the proof of Proposition 10.1.) Let $\mathcal{P}_{n}$ (or $P_{n}^{a}$ ) be the components of the complement of $g^{-n} A$. This forms a natural dynamical partition of the real line. Since $g(A) \subset A$, any component of $\mathcal{P}_{n+1}$ is contained in one and only one component of $\mathcal{P}_{n}$. Let $P_{n}(t)$ be the element of the partition which contains $t$ (this is well-defined if $t$ is not eventually mapped into $A$; if it is, take the interval whose interior is not contained in the basin of a periodic attractor and also does not intersect a gap between two domains of $g$ ). If two domains of $g$ have a common end point then there is a choice, but then it will be clear from the context which interval $P_{n}(t)$ one should take. Since $g$ has no wandering intervals, for each $t \in \omega(c)$, the diameter of $P_{n}(t)$ tends to zero as $n \rightarrow \infty$.

In this section we shall prove the following theorem:

Theorem A' (complex bounds in the recurrent non-minimal case). Let $g \in \mathbf{A C}$ and assume that $\omega(c)$ is recurrent and non-minimal. Then $g$ induces a Box Mapping: there exists a sequence $C_{k} \rightarrow \infty, \theta$ close to zero, and a sequence of open topological discs $V_{k}:=D\left(V_{k} \cap \mathbb{R}, \pi-\theta\right)$ around the critical point such that the following properties hold.

1. $\operatorname{diam}\left(V_{k}\right) \rightarrow 0$ and $V_{k} \cap \mathbb{R}$ is a critical piece of the above partition.

2. the (complex analytic) first return map to $V_{k}$ restricted to domains containing points of $\omega(c)$ is a box mapping $F_{k}: \cup_{i} V_{k}^{i} \rightarrow V_{k}$ (see the definition below the statement of the theorem).

3. the modulus of the annulus $V_{k} \backslash V_{k}^{i}$ is bounded from below by $C_{k}$.

Passing to the first return map to the central domain $V_{k}^{0}$, we then obtain again a box mapping $F_{k}: \cup_{i} V_{k}^{i} \rightarrow V_{k}$ (where this time $V_{k}$ is the central domain $V_{k}^{0}$ and were the domains $V_{k}^{i}$ are the new domains) with all listed properties, and additionally such that:

4. each branch $F_{k} \mid V_{k}^{i}$ extends to a univalent map $F_{k} \mid W_{k}^{i}$ for $i \neq 0$ (and for $i=0$ to an analytic covering map which is branched at $c)$, such that the modulus of $W_{k}^{i} \backslash V_{k}^{i}$ is at least $C_{k}$, and such that for $i \neq j$ either $W_{k}^{i}$ is contained in $W_{k}^{j} \backslash V_{k}^{j}$ or the other way around $W_{k}^{j}$ is contained in $W_{k}^{i} \backslash V_{k}^{i}$ (or $W_{k}^{i}$ and $W_{k}^{j}$ are disjoint). Moreover, $W_{k}^{i}$ is contained in $V_{k}$ for each $i$. 
Here we say that $F_{k}: \cup_{i} V_{k}^{i} \rightarrow V_{k}$ is a box mapping (following terminology of [GS2]) if the countably many domains $V_{k}^{i}, V_{k}^{j}$ with $i \neq j$ are pairwise disjoint, $V_{k}^{i}$ is contained in $V_{k}$ for all $i$ and if $F_{k} \mid V_{k}^{i} \rightarrow V_{k}$ is a diffeomorphism for $i \neq 0$ while $F_{k} \mid V_{k}^{0} \rightarrow V_{k}$ is a $\ell$-branched covering.

It is very easy to prove an analogous theorem in the non-recurrent case, see [Str] and [MS]. In that case all branches are diffeomorphic (i.e. $V_{k}^{0}$ simply does not exist).

Proof: The proof uses ideas of Proposition 3.3 from [LS1]. Assume that $\omega(c)$ is recurrent and non-minimal. For each integer $i$ one has $\left|P_{n}\left(g^{i}(c)\right)\right| \rightarrow 0$ as $n \rightarrow \infty$. First, let us show the existence of a sequence of (real) critical pieces $P_{n_{k}}(c)$, such that for the (real) central component $U_{k}$ of the first return map to $P_{n_{k}}(c)$, we have: $C^{-1}\left(P_{n_{k}}(c), U_{k}\right) \rightarrow \infty$ as $k \rightarrow \infty$. Since $\omega(c)$ is non-minimal, there exists $x \in \omega(c)$ such that forward iterates of $x$ avoid some neighbourhood of the critical point. Therefore, there exists $N$ such that for an infinite sequence of $k$, then we choose the interval $P_{k}(x) \in \mathcal{P}_{k}$ so that it is mapped by $g^{k-N}$ diffeomorphically onto some fixed interval $P:=P_{N} \in \mathcal{P}_{N}$ contained in one of the domains of $g$ and such that $g^{i}\left(P_{k}(x)\right)$ does not contain $c$ for $i=0,1, \ldots, k-N-1$. Considering the first entry of $c$ into $P_{k}(x)$, we then obtain a sequence of intervals $P_{n_{k}}(c)$ with diameters shrinking to zero and integers $n_{k} \rightarrow \infty$ so that $g^{n_{k}-N}: g\left(P_{n_{k}}(c)\right) \rightarrow P$ is a diffeomorphism. Fix $k$ for a moment. By the construction, the iterates $g(c), \ldots, g^{n_{k}-N-1}(c)$ are outside $P_{n_{k}}(c)$. Let $s>n_{k}-N-1$ be the smallest integer for which $g^{s}(c) \in P_{n_{k}}(c)$. Let $U_{k}$ be the pullback of $P_{n_{k}}(c)$ from $g^{s}(c)$ to $c$.

Claim 1: The interval $g^{n_{k}-N}\left(U_{k}\right)$ is very deep inside $P$, i.e., $C^{-1}\left(P, g^{n_{k}-N}\left(U_{k}\right)\right) \rightarrow \infty$ as $k \rightarrow \infty$.

Proof of claim 1: The length of $g^{s}\left(U_{k}\right)=P_{n_{k}}(c)$ tends to zero as $k \rightarrow \infty$ and therefore the length of $g^{n_{k}-N}\left(U_{k}\right)$ tends to zero as $k \rightarrow \infty$ (here we use that $n_{k}-N<s$ and that $g$ has no wandering intervals). If $\partial P$ is a preimage of a repelling periodic point, then we can go to big scale with bounded distortion, and so if $g^{n_{k}-N}\left(U_{k}\right)$ is close to the boundary of $P$ then $g^{n_{k}-N}\left(U_{k}\right)$ is very deep inside $P$ (if it is not close to one of the boundary points there is nothing to prove).

But in fact, we do not even need to use that $\partial P$ is in a hyperbolic repellings set. Indeed, let $\partial P$ be a preimage of the periodic point $a$, which is parabolic, and assume that again $g^{n_{k}-N}\left(U_{k}\right)$ is close to a boundary point $x$ of $P$, but the distance between $g^{n_{k}-N}\left(U_{k}\right)$ and $x$ is comparable to the length of $g^{n_{k}-N}\left(U_{k}\right)$. Then, when one end point of $g^{n_{k}-N}\left(U_{k}\right)$ goes to a fixed (large) distance from $a$ by an iterate $g^{r}$, then the length of $g^{r}\left(g^{n_{k}-N}\left(U_{k}\right)\right)$ is also large (i.e., independent of $k$ ). (We see this in a standard local coordinate at the parabolic point.) But this is impossible, because $g^{s}\left(U_{k}\right)$ tends to zero.

Claim 2: $C^{-1}\left(P_{k}(x), g^{n_{k}-k}\left(U_{k}\right)\right)$ tends to infinity as $k \rightarrow \infty$.

Proof of Claim 2: The proof of this is essentially the same as that of Claim 1. Indeed, $g^{k-N}$ maps $P_{k}(x)$ diffeomorphically onto $P \in \mathcal{P}_{N}$. If $\omega(x)$ does not contain parabolic periodic points, then $x$ is contained in a hyperbolic set because of Mañé's theorem 2.2. So in this case $g^{k-N}$ has bounded distortion on $P_{k}(x)$ and the proof follows immediately 
from Claim 1. If $\omega(x)$ contains a parabolic periodic point, then we can assume that $x$ is one of these (finitely many) parabolic periodic points, and $g^{n_{k}-k}$ simply maps in interval $P_{k}(x)$ periodically along the parabolic periodic orbit $O(x)$ to the big interval $P$ (where $P$ also contains a point from $O(x)$ as a boundary point by the construction of the partition). By the previous claim, both components of $P \backslash g^{n_{k}-k}\left(U_{k}\right)$ contain a very large number of adjacent fundamental domains of the parabolic periodic orbit $O(x)$. But then the same holds for the components of $P_{k}(x) \backslash g^{n_{k}-k}\left(U_{k}\right)$. Since neighbouring fundamental domains of a parabolic periodic point have approximately the same size, it follows that $g^{n_{k}-k}\left(U_{k}\right)$ is very deep in $P_{k}(x)$. This concludes the proof of Claim 2.

The intervals $g^{i}\left(P_{n_{k}}(c)\right), 0 \leq i \leq n_{k}-k$, are disjoint, because $g^{n_{k}-k}\left(P_{n_{k}}(x)\right)$ is the first visit of some iterate of $P_{n_{k}}(c)$ to $P_{k}(x)$. Hence we can apply the Koebe Principle to estimate the cross-ratio and obtain $C^{-1}\left(P_{n_{k}}(c), U_{k}\right)$ tends to $\infty$ as $k \rightarrow \infty$. To prove the theorem, fix now $k$ and consider the first return map to $P_{n_{k}}:=P_{n_{k}}(c)$. Then each (and not only the central) component of this map is very deep inside $P_{n_{k}}$ (we apply the margins disjointness property (4.1) this time which holds because the components of the first return map are disjoint). Define $V_{k}=D\left(P_{n_{k}} ; \pi-\theta\right)$, where $\theta$ is small enough, and consider the pullback of $V_{k}$ along some branch of the latter first return. Because we consider first returns, the sum of the lengths of the preimages of $P_{n_{k}}$ along this pullback is uniformly bounded (by the length of the range of $g$ ). Hence choosing $\theta$ small enough, we obtain by Lemma 2.3, that the pullback is well-defined and is contained deep inside $V_{k}$. Disjointness of the domains holds because by Lemma 2.3 the $g$-images of all the domains (which are near $g(c))$ are contained in sets of the form $D\left(g\left(V_{k}^{i}\right) \cap \mathbb{R} ; \pi-\theta^{\prime}\right.$ ) with $\theta^{\prime}>0$ small. This implies the first three assertions of the theorem.

In property 4 the range of the extension can be taken to be the range of the previous map.

It will be useful later on to modify the range of the polynomial-like map constructed in Theorem A'. The reason for this is that later on we will consider complex perturbations of the map. Therefore we will want to modify the range of the polynomial-like map in a more dynamical way (to deal with complex eigenvalues), so that nearby maps still have an associated polynomial-like map.

Addendum to Theorem A': Assume that $\omega(c)$ contains no parabolic periodic points; in particular, assume that the periodic points in the set $A$ are hyperbolic. Then, given $k$, we can modify the range $V=V_{k}=D\left(P_{n_{k}} ; \pi-\theta\right)$ in the following way (imitating in some sense external rays to periodic points in the standard Yoccoz puzzle construction). Let us do this construction in the case that $a \in A$ is a fixed point (the periodic case goes in the same way).

1. Denote $\sigma=g^{\prime}(a)$. Then $\sigma>1$ and the branch $g^{-1}$ (so that $g^{-1}(a)=a$ ) is conformally conjugate in a fixed neighbourhood $Z$ of $a$ to the map $w \mapsto \sigma^{-1} w$. It follows that, given a unit vector $v$ at the point $a$ there exists a unique (analytic) curve $l_{v}$ in $Z$ which starts at the point $a$ and so that $v$ is the tangent vector of $l_{v}$ at $a$, such that $g^{-1}\left(l_{v}\right) \subset l_{v}$. It gives a foliation of $Z \backslash\{a\}$.

2. Fix two neighbourhoods $Z_{1}, Z_{2}$ of the end points $a_{1}, a_{2}$ of the real trace of $V$, 
such that $g^{M}: Z_{i} \rightarrow Z, i=1,2$, are well-defined and injective. For $b_{i} \in \partial V \cap Z_{i}$ let $l_{v_{i}}$ be the leaf of the foliation which contains $g^{M}\left(b_{i}\right)$. If we choose point $b_{i} \in \partial V \cap Z_{i}$ sufficiently close to $a_{i}$, then the vector $v_{i}$ is close to one of the two (complex conjugate) tangent vectors of $g^{M}\left(\partial V \cap Z_{i}\right)$ at $a$ and also the end point of $l_{v_{i}}$ (other than $a$ ) is non-real.

3. Replace the small arc in $\partial V$ connecting $b_{i}$ and $a_{i}$ by $g^{-M}\left(l_{v}\right)$ (and do the same for its complex conjugate). We obtain in this way a boundary of some domain $\tilde{V}$

Then the domain $\tilde{V}$ can be chosen instead of $V$ in the construction of the box mapping. Indeed, $\partial \tilde{V}$ is contained between two domains $D\left(P_{n_{k}} ; \pi-\theta_{-}\right)$and $D\left(P_{n_{k}} ; \pi-\right.$ $\left.\theta_{+}\right)$, where $\theta_{ \pm}$are close to $\theta$. Hence, all the pullbacks are well-defined and form a box mapping just as in Theorem A'.

We shall need also the following.

Proposition 10.1 (Construction of Cantor repeller) Assume that $g$ is as in the statement of Theorem $A$ ' and in addition assume that $\omega(c)$ contains no parabolic periodic points. Fix a critical piece $P:=P_{m}^{a}(c)$ from the partition defined above, and let $F: \cup_{i} P^{i} \rightarrow P$ be the restriction of the first return map to $P$ to domains containing points of $\omega(c) \cap P$. Let $X$ be the closure of the end points of $P^{i}$. Then there exists a hyperbolic Cantor set $\hat{X} \supset X$ which is contained in the set of non-escaping poirits ( $a$ Cantor repeller) of some 'polynomial-like mapping'

$$
G: \cup B(J) \rightarrow \cup D(I ; \pi-\beta),
$$

(without critical points and with a finite number of different ranges)

Proof: Here $P^{i}$ are (some of the) pieces of the partition $\mathcal{P}^{a}$ inside $P$, and, as usual, $P^{0}$ is the critical one. Observe that the forward iterates (by $g$ ) of the points $x \in X$ avoid a neighbourhood $U$ of the parabolic cycles of $g$, because $\omega(c)$ is a closed set not containing such cycles, $P^{i}$ are pieces covering $\omega(c) \cap P$, and $\partial P^{i}$ are pre-images of points $a \in A$. Define $\hat{X}$ to be the set of points $y$ from the domain of $g$ such that all iterates $g^{j}(y), j \geq 0$ are well-defined and lie outside the piece $P^{0}$ and outside the neighbourhood $U$ of the parabolic cycles and outside the basins of attraction. Note that $X \subset \hat{X}$.

Let us now choose a repelling periodic point $b$ inside the central piece $P^{0}$ and let $B=O(b)$. We would like to construct a partition using the backward orbit of $B$. However, preimages of $B$ do not (necessarily) accumulate from both sides to points from $A$. There are several ways to remedy this. For example, consider the extension $\bar{g}: S^{1} \rightarrow S^{1}$ of $g$ from assumption 4 in the definition of the class AC. Modify $g$ possibly even further on neighbourhoods of periodic attractors (and parabolic points) by gluingin additional branches inside immediate basins. By choosing the derivative large this can be done so that for the new map $\tilde{g}: S^{1} \rightarrow S^{1}$ the points from the set $A$ are no longer in the basins of periodic attractors. Then simply let $\mathcal{P}_{n}^{b}$ be the partition generated by $\tilde{g}^{-n}(B)$. It is absolutely crucial to observe that we shall actually only consider elements 
of the partition on which the $C^{\infty}$ map $\tilde{g}$ coincides with the real analytic map $g$. We do this by choosing $n_{0}$ so large that $\tilde{g}$ coincides with $g$ on each element of the partition $\mathcal{P}_{n_{0}}^{b}$ containing points of the set $\hat{X}$. Fix $n_{0}$ also so large that the critical piece $P_{n_{0}}^{b}(c)$ of this partition is completely inside the critical piece $P^{0}$ of the previous partition (determined by the set $A$ ).

One can assume also that the neighbourhood $U$ of the parabolic cycles of $g$ consists of finitely many pieces of $\mathcal{P}_{n_{0}}^{b}$. The set $\hat{X}$ is compact (because $g$ has finitely many domains of definition) and forward invariant under $g$ and a positive distance away from the end points of the pieces of $\mathcal{P}_{n_{0}}^{b}$ (since these end points hit $b \in P^{0}$ eventually). For every $n>n_{0}$, consider the elements of the partition $\mathcal{P}_{n}^{b}$ which intersect $\hat{X}$. Denote these intervals by $J_{n}^{k}$. Note that for $n$ large, the remaining (finitely many) pieces have a definite distance to the boundary of the pieces of the finite partition $\mathcal{P}_{n_{0}}^{u}$. Let us choose a sufficiently small angle $\beta>0$, and conșider Poincaré neighbourhoods $D\left(I^{i} ; \pi-\beta\right)$, where $I^{i}$ are the elements of the partition $\mathcal{P}_{n_{0}}^{b}$ except for the critical piece $P_{n_{0}}^{b}(c)$ of this partition. Take $n>n_{0}$ and any interval $J=J_{n}^{k}$. Then $g^{n-n_{0}}(J)$ is equal to some interval $I^{i(J)}$ (we write $I^{i(J)}$ to emphasize that the interval depends on $J$ ), and moreover the iterates $g^{j}(J), j=0,1, \ldots, n-n_{0}$ are all disjoint from the piece $P_{n_{0}}^{b}(c)$ and from the neighbourhood $U$ of the parabolic points. Hence, by Mañés theorem 2.2, the sum of the lengths of these intervals is bounded by a universal constant, and, as we know (for any $n$ large and $\beta$ small fixed), there exists a domain $B(J)$, such that the map $g^{n-n_{0}}: B(J) \rightarrow D\left(I^{i(J)} ; \pi-\beta\right)$ is well-defined, $B(J)$ is inside $D(J ; \pi-C \beta)$, where $C>0$ is some universal constant. The closed set $\hat{X}$ is contained in the (finite) union of the interiors of $I^{i}$. It follows that as $n \rightarrow \infty$, each of the intervals $J$ (the partition elements of $\mathcal{P}_{n}^{b}$ intersecting $\hat{X}$ ) becomes deeper and deeper inside the (appropriate) interval $I^{i}$. Therefore, provided $n$ is large enough, the domains $B(J)$ are well inside the domains $D\left(I^{i} ; \pi-\beta\right)$ and pairwise disjoint.

Thus we end up with a map

$$
G: \cup B(J) \rightarrow \cup D(I ; \pi-\beta),
$$

where $J$ runs over the finitely many intervals $J_{n}^{k}$ (from $\mathcal{P}_{n}^{b}$ ) and $I$ runs over the finitely many intervals $I^{i}$ (i.e, every piece of $\mathcal{P}_{n_{0}}^{b}$ except the central piece one). If two domains $B(J)$ touch then we shrink $D(I ; \pi-\beta)$ slightly, and then the new domains $B(J)$ will no longer touch. The above map is a polynomial-like map (without critical points and with a finite number of different ranges).

Note that $X \subset \hat{X}$, and that points from $\hat{X}$ never leave the domain of definition of $G$ under iterates of $G$. 


\section{Proof of Theorem B': quasisymmetric rigidity of maps in the non-recurrent and the non-minimal case}

\section{Theorem B' (rigidity)}

Consider two maps of the class $\mathbf{A C}$ with critical points of the same order, such that for both maps $c$ is not attracted to an attracting or parabolic periodic orbit, $\omega(c)$ is nonminimal or non-recurrent, and contain no parabolic periodic points. If the conjugacy on $\omega(c)$ maps the critical point of one map to the critical point of the other map, then the conjugacy is quasisymmetric on $\omega(c)$.

Proof: In the non-recurrent case, this is an exercise from Section III.6.2 of [MS]. So let us assume that we are in the recurrent non-minimal case. Fix maps $g, \tilde{g}$ as in Theorem B'. Denote the box mapping from Theorem A' by

$$
F: \cup_{i=0}^{\infty} \Omega_{i} \rightarrow \Omega \text { and } \tilde{F}: \cup_{i=0}^{\infty} \tilde{\Omega}_{i} \rightarrow \tilde{\Omega}
$$

By assumption, $F$ and $\tilde{F}$ are conjugate on $\omega(c) \cap \Omega$, such that the conjugacy maps the critical point of $F$ to that of $\tilde{F}$. Hence, one can choose the generating real partitions in the construction of the box mappings (see Section 10 ) in such a way that $F, \tilde{F}$ are the same iterate of $g, \tilde{g}$ on corresponding components. In what follows, $\tilde{\Omega}_{i}$ denotes the component of $\tilde{F}$ which corresponds to the component $\Omega_{i}$ of $F$. Let us try to extend the conjugacy on $\omega(c)$ to a quasiconformal conjugacy between $F$ and $\tilde{F}$, as in [LS1]. The problem is that in this case we do not apriori have a natural external conjugacy, i.e., a quasiconformal map from

$$
A:=\operatorname{closure}\left(\Omega \backslash \cup_{i=0}^{\infty} \Omega_{i}\right)
$$

onto

$$
\tilde{A}:=\operatorname{closure}\left(\tilde{\Omega} \backslash \cup_{i=0}^{\infty} \tilde{\Omega}_{i}\right),
$$

which respects the dynamics of $F$ and $\tilde{F}$ on the boundaries. (We can assume that $F$ and $\tilde{F}$ are defined dynamically on the closures of $\Omega_{i}$ and $\tilde{\Omega}_{i}$.) In the case of polynomials or (generalized) polynomial-like mappings such external conjugacy always exists: it is a composition of the Böttcher functions at the basins of infinity or external maps $\mathrm{DH}]$. Then all steps of the proof below are greatly simplified. In the general case we will proceed as follows.

In Steps 1-2 we construct a quasiconformal external conjugacy $H: A \rightarrow \tilde{A}$. In Step 3 we construct extensions of $H: A \rightarrow \tilde{A}$ using a pullback construction and describe some of the geometric structure involved. Finally, in Step 4 we consider the dynamical extension of $H$ to a map $\cup_{n=0}^{\infty} F^{-n}(A) \rightarrow \cup_{n=0}^{\infty} \tilde{F}^{-n}(\tilde{A})$ (this is trivial, just pullback dynamically), and then show that this map can be extended quasiconformally to $\Omega$ as a conjugacy.

The box mapping $F$ is one of the box mappings $F_{k}$ of Theorem A'. We will fix $k$ and therefore drop $k$ in the notation. In particular, we denote by $W_{i}=W_{k}^{i}$ the neighbourhood of $\Omega_{i}=\Omega_{k}^{i}$, and by $C=C_{k}$ the constant from property (4) of Theorem 
A'. To simplify the picture, we choose the map $F$ so that the constant $C$ is so large that every annulus $W_{i} \backslash \Omega_{i}$ contains a standard round annulus surrounding $\Omega_{i}$ with the modulus at least $C / 2$. Similar for $\tilde{F}$.

Given a set $Y$ of the plane, denote $Y^{+}=\{z \in Y ; \Re(z) \geq 0\}$ and $Y^{-}=\{z \in$ $Y ; \Re(z) \leq 0\}$.

Step 1: In this step, we shall show that the boundary of each of the sets $A^{ \pm}$(and similarly for $\tilde{A}$ ) is a quasi-circle using the Ahlfors-Beurling criterion [LeVi]. Pick any two points $z_{1}, z_{2}$ in, say, $\partial\left(A^{+}\right)$, and consider one of the two subarcs $C=C\left(z_{1}, z_{2}\right)$ of $\partial\left(A^{+}\right)$between $z_{1}$ and $z_{2}$ of a smaller diameter. According to the Ahlfors-Beurling criterion, we need to prove the existence of a constant $L$ independent of $z_{1}, z_{2}$ such that $C$ lies inside a circle of diameter $L \cdot\left|z_{1}-z_{2}\right|$. Let us consider several possibilities for $z_{1}$ and $z_{2}$. (a) If $z_{1}, z_{2}$ are both in the boundary of either $\Omega$ or some $\Omega_{i}$, such $L$ exists (which does not depend on $\Omega_{i}$ ). Indeed, $L$ exists for $\Omega$, because $\partial \Omega$ is a piecewise analytic curve without turning points. For the same reason, $L$ exists for the central component $\Omega_{0}$. Any other $\Omega_{i}$ is an image of $\Omega$ under a branch of $F^{-1}$, which is univalent in a fixed neighbourhood $W$ of $\Omega$. Thus $L$ is independent on $i$. (b) If $z_{1}, z_{2}$ are in the boundaries of different $\Omega_{i}, \Omega_{j}$, then we notice that from Theorem A' one gets that the diameter of these topological discs is comparable to their real trace, that the discs are roughly round (cf. Fact 11.3 (1d) below). This and the case (a) imply that one has a uniform choice for $L$ in this case. (c) These considerations also settle the case when one or both points $z_{1}, z_{2}$ are real, or belong to $\partial \Omega$.

Thus $\partial\left(A^{ \pm}\right)$and similarly $\partial\left(\tilde{A}^{ \pm}\right)$are quasi-circles. So there exist $K$-quasiconformal homeomorphisms $\phi^{ \pm}: D \rightarrow A^{ \pm}, \tilde{\phi}^{ \pm}: D \rightarrow \tilde{A}^{ \pm}$, where $D$ stands for the unit disc. We choose $\phi^{ \pm}$to be so that $\overline{\phi^{+}(z)}=\phi^{-}(\bar{z})$ (and similarly for $\tilde{\phi}$ ).

Step 2: In this step we show that there exists a quasiconformal conjugacy $H$ : $A \rightarrow \tilde{A}$.

Note that the boundary $\partial\left(A^{+}\right)$of $A^{+}$consists of the set $(\partial A)^{+}$and some (possibly empty) open subset $O$ of $\bar{\Omega} \cap \mathbb{R}$ (for example, $O$ can contain an open set of points which are attracted by an attracting periodic orbit of $g$ ).

We are going to construct the map $H: \partial A \rightarrow \partial \tilde{A}$ to be symmetric (i.e. $\overline{H(z)}=$ $H(\bar{z}))$ and so that $H \mid(\partial A)^{+}$is a restriction of some map $H^{+}: \partial\left(A^{+}\right) \rightarrow \partial(\tilde{A})^{+}$to the subset $(\partial A)^{+}$of $\partial\left(A^{+}\right)$. Thus we need to construct $H^{+}$(such that $H^{+}(x)$ is real for $x$ real). Then $H^{-}: \partial\left(A^{-}\right) \rightarrow \partial\left(\tilde{A}^{-}\right)$is defined symmetrically: $H^{-}(\bar{z})=\overline{H^{+}(z)}$, so that $H(z)=H^{+}(z)$ iff $\Re(z) \geq 0$ and $H(z)=H^{-}(z)$ iff $\Re(z) \leq 0$.

First define $H^{+}:(\partial \Omega)^{+} \rightarrow(\partial \tilde{\Omega})^{+}$as follows. For both endpoints $y$ of $\Omega \cap \mathbb{R}$ fix a neighbourhood $Y$ such that some iterate $g^{N}$ maps $Y$ univalently onto the neighbourhood $\underset{\tilde{Z}}{Z}$ of the fixed point $a$ of $g$ (see Addendum to Theorem A'). Similar for $\tilde{F}$ : $\tilde{g}^{N}: \tilde{Y} \rightarrow \tilde{Z}$. By the construction of $\Omega, g^{N}\left((\partial \Omega \cap Y)^{+}\right)=l_{v}^{+}$and $\tilde{g}^{N}\left((\partial \tilde{\Omega} \cap Y)^{+}\right)=\tilde{l}_{v}^{+}$, where $l_{v}, \tilde{l}_{v}$ are invariant arcs from Addendum to Theorem A'. Now fix a quasiconformal symmetric conjugacy $K$ between $g \mid Z$ and $\tilde{g} \mid \tilde{Z}$, such that $K\left(l_{v}\right)=\tilde{i}_{v}$. Then define $H^{+}$on the $\operatorname{arc} g^{-N}\left(l_{v}^{+}\right)=(\partial \Omega \cap Y)^{+}$of $\partial \Omega$ as $H^{+}=\tilde{g}^{-N} \circ K \circ g^{N}$. Extend $H^{+}$to the remainder of $(\partial \Omega)^{+}$so that it distorts (Euclidean) distances by at most a uniformly bounded factor.

Next, define $H^{+}$on each $\left(\partial \Omega_{i}\right)^{+}$dynamically as follows. For $z \in\left(\partial \Omega_{i}\right)^{+}$, let 
$H^{+}(z)=\tilde{F}_{\mid \tilde{\Omega}_{i}}^{-1} \circ H^{ \pm} \circ F_{\mid \Omega_{i}}(z)$, where $H^{ \pm}=H^{+}$iff $F(z) \in(\partial \Omega)^{+}$and $H^{ \pm}==H^{-}$ iff $F(z) \in(\partial \Omega)^{-}$. It is clear also which branch of $\tilde{F}_{\Omega_{0}}^{-1}$ to take on the central domain. If $n$ is such that $g^{n}\left(\Omega_{i}\right)=\Omega$, then extend $H_{\mid g^{k}\left(\partial \Omega_{i}\right)^{+}}^{+}=\tilde{g}^{-(n-k)} \circ H_{(\partial \Omega)^{+}}^{+} \circ g^{n-k}$, $k=1, \ldots, n-1$.

Finally, the remainder of $(\partial A)^{+}$is the set $X \cup O$ on the real line, where $X$ is the Cantor set introduced in Proposition 10.1. Remember that $X \subset \hat{X}$, where $\hat{X}$ is the Cantor set on the real axis invariant under $g$ and is contained in the set of non-escaping points of the expanding map $G: \cup B(J) \rightarrow \cup D(I ; \pi-\beta)$ (without critical points and with a finite number of different ranges). Let $\tilde{X}, \hat{\hat{X}}, \tilde{G}: \cup B(\tilde{J}) \rightarrow \cup D(\tilde{I} ; \pi-\beta)$ be the corresponding sets and the map of $\tilde{F}$ chosen so that $G$ and $\tilde{G}$ are combinatorially conjugate (it is done by constructing $G$ and $\tilde{G}$ as in the proof of Proposition 10.1 simultaniously for $F$ and $\tilde{F}$ in a combinatorially the same way). Then $G, \tilde{G}$ are quasiconformally conjugate: there exists a quasiconformal symmetric map $\tau: \cup D(I ; \pi-\beta) \rightarrow \cup D(\tilde{I} ; \pi-\beta)$ such that $\tau \circ G=\tilde{G} \circ \tau$ on $\cup B(J)$. In particular $\tau(X)=\tilde{X}$. One can assume that $\tau$ is extended to a quasiconformal map of the plane. Define finally $H^{+}=\tau$ on $X \cup O$. This completes the definition of $H^{+}: \partial\left(A^{+}\right) \rightarrow \partial\left(\tilde{A}^{+}\right)$.

Let us now show that the map $\left(\tilde{\phi}^{+}\right)^{-1} \circ H^{+} \circ \phi^{+}: \partial D \rightarrow \partial D$ is quasisymmetric. In order to do this, take three nearby points $z_{1}, z_{2}, z_{3} \in \partial D$ with $z_{3}$ the midpoint between $z_{1}$ and $z_{2}$. Then the points $w_{i}=\phi^{+}\left(z_{i}\right) \in \partial\left(A^{+}\right), i=1,2,3$, are roughly equidistant because $\phi^{+}$is quasiconformal. ("Roughly equidistant" means that the distances between the points are roughly the same; we say that numbers $a, b$ are roughly the same: $a \asymp b$ (resp. $a$ is much bigger than $b$ ) if $L^{-1} \leq a / b \leq L$ (resp. $a / b \geq l$ ), for some universal $L>1)$.

We need to show that the points $\tilde{w}_{i}=H^{+}\left(w_{i}\right), i=1,2,3$, are roughly equidistant as well.

Assume $w_{1}, w_{2}, w_{3}$ all lie on the real line or all belong to the boundary of either $\Omega$ or of some $\Omega_{k}$. Then we are done because $H^{+}$is quasisymmetric on the real line, distorts distances by a bounded factor on $\partial \Omega$, and the map $F: \Omega_{i} \rightarrow \Omega$ has definite Koebe space.

Hence, we can assume that at least oile and at most two of the points $w_{i}$ lie in one of the domains $\partial \Omega_{k}$. Denote by $B_{i}, i=1,2,3$, the domain $\Omega_{j}$ of $F$ for which $w_{i} \equiv \partial B_{i}$ (for example, one of the sets $B_{i}$ is $\Omega_{k}$; if $w_{i}$ is outside all the domains $\Omega_{j}$, then $w_{i} \in \mathbb{R}$ and we take $B_{i}$ to be the point $\left.w_{i}\right)$. Let $U_{i}(x)$ be a neighbourhood of an endpoint $x$ of $B_{i} \cap \mathbb{R}$, such that $F\left(U_{i}(x)\right)=Y$, where $Y$ is the above mentioned neighbourhood of one of the endpoints of $\Omega \cap \mathbb{R}$ from the domain of definition of $H_{\mid(\partial \Omega 2)^{+}}^{+}$. There are two main cases to distinguish.

(a) all points $w_{i}$ lie in $U_{j}(x)$, for some $j$ between 1 and 3 . Then pass to big scale. The map $g^{N} \circ F=g^{N_{1}}: U_{j}(x) \rightarrow Z$ has bounded distortion, and $Z$ is the neighbourhood of the repelling fixed point $a$ of $g$. Then the point $z_{j}=g^{N_{1}}\left(w_{j}\right)$ lies in the arc $l_{v}^{+}$. So we can iterate the map $g$ (keeping bounded distortion) until the distances of images of $w_{i}$ become larger than a universal positive number. (We use that there is a universal $\epsilon>0$ such that the Euclidean distance between any two components $\Omega_{i}, \Omega_{j}$ is larger than $\epsilon \cdot\left\{\right.$ minimal diameter of $\left.\Omega_{i}, \Omega_{j}\right\}$, see Fact 11.3 for detail; the same holds for the images of $\Omega_{i}, \Omega_{j}$ until one of the images is $\Omega$. Hence, $g^{N_{1}}\left(B_{i}\right), i \neq j$, lie in a $(1+\epsilon)$ - 
neighbourhood of $Z$, i.e. still close to the fixed point $a$ ). Hence we can transport all this by the map $K$, and then by an inverse to $\tilde{g}^{N_{1}}$, and get again that the property that these three points are roughly equidistant is preserved.

(b) for every $j=1,2,3$, at least one of the points $w_{1}, w_{2}, w_{3}$ is outside the neighbourhood $U_{j}(x)$. To avoid to deal with many scales and cases (diameters of $B_{1}, B_{2}, B_{3}$ and mutual distances between $\left.B_{i}\right)$, we do the following. Remember that $H_{\mid \partial\left(A^{+}\right) \cap \mathbb{R}}^{+}$ is the restriction of the quasiconformal map $\tau$ of the plane. Hence, the points $\tilde{w}_{i}^{\prime}=$ $\tau\left(w_{i}\right), i=1,2,3$, are roughly equidistant, and we finish the proof by showing that for every $i, j$, the distances $\tilde{l}=\left|\tilde{w}_{i}-\tilde{w}_{j}\right|$ and $\tilde{l}^{\prime}=\left|\tilde{w}_{i}^{\prime}-\tilde{w}_{j}^{\prime}\right|$ are roughly the same: $\tilde{l} \asymp \tilde{l}^{\prime}$. Let $\tilde{B}_{i}$ be the component of $\tilde{F}$ with $\partial \tilde{B}_{i}=H^{+}\left(\partial B_{i}\right)$, and $\tilde{B}_{i}^{\prime}=\tau\left(B_{i}\right)$. Denote also $d_{i}, \tilde{d}_{i}, \tilde{d}_{i}^{\prime}$ the diameters of $B_{i}, \tilde{B}_{i}, \tilde{B}_{i}^{\prime}$, and $D, \tilde{D}, \tilde{D}^{\prime}$ the distances between $B_{i}$ and $B_{j}, \tilde{B}_{i}$ and $\tilde{B}_{j}$, $\tilde{B}_{i}^{\prime}$ and $\tilde{B}_{j}^{\prime}$ respectively. Consider three simple subcases. (1) if $\max \left(d_{i}, d_{j}\right) \asymp D$, then all numbers $\max \left(\tilde{d}_{i}, \tilde{d}_{j}\right), \tilde{D}, \max \left(\tilde{d}_{i}^{\prime}, \tilde{d}_{j}^{\prime}\right), \tilde{D}^{\prime}$ are roughly the same (because the real traces of $B_{i}, \tilde{B}_{i}, \tilde{B}_{i}^{\prime}$ are comparable with their diameters, and the map $\tau$ is quasisymmetric on $\mathbb{R})$. Therefore $\tilde{l}, \tilde{l}^{\prime}$ are roughly equal to $D ;(2)$ if $d_{i}$ and $d_{j}$ are much smaller than $D$, the same holds for $\tilde{d}_{i}, \tilde{d}_{j}$ and $\tilde{D}$, and for $\tilde{d}_{i}^{\prime}, \tilde{d}_{j}^{\prime}$ and $\tilde{D}^{\prime}$. But $\tilde{D} \asymp \tilde{D}^{\prime}$, hence, $\tilde{l}, \tilde{l}^{\prime}$ are roughly $\tilde{D} ;(3) d_{i}$ is much bigger than $d_{j}$ and than $D$. Let $x$ be an endpoint of $B_{i} \cap \mathbb{R}$ closer to $B_{j}$. The neighbourhood $U_{i}(x)=F_{\mid B_{i}}^{-1}(Y)$ is roughly a round disc, and the diameters of $U_{i}(x)$ and $B_{i}$ are roughly the same. Hence, there exists a round disc $U^{\prime}(x)$ inside $U_{i}(x)$ and centered at $x$, with radius roughly $d_{i}$, such that $w_{i} \notin U^{\prime}(x)$, because otherwise all points $w_{1}, w_{2}, w_{3}$ would be in $U_{i}(x)$. Hence $l=\left|w_{i}-w_{j}\right| \asymp d_{i}$. Also, $\tilde{w}_{i}^{\prime} \notin \tau\left(U^{\prime}(x)\right)$, hence $\tilde{l}^{\prime} \asymp \tilde{d}_{i}^{\prime}$. Moreover, since $\tilde{w}_{i}=\tilde{F}^{-1} \circ H_{\mid(\partial \Omega)^{+}}^{+} \circ F\left(w_{i}\right)$, the point $\tilde{w}_{i}$ is outside a definite neighbourhood of $\tilde{x}=H^{+}(x)$, and again $\tilde{l} \asymp \tilde{d}_{i}$. But $\tilde{d}_{i} \asymp \tilde{d}_{i}^{\prime}$ (they are comparable with common real trace of $\tilde{B}_{i}, \tilde{B}_{i}^{\prime}$ ), and we are done.

Thus $\left(\tilde{\phi}^{+}\right)^{-1} \circ H^{+} \circ \phi^{+}: \partial D \rightarrow \partial D$ is quasisymmetric, and therefore there exists a quasiconformal extension $H^{+}$: $\operatorname{closure}\left(A^{+}\right) \rightarrow \operatorname{closure}\left(\tilde{A}^{+}\right)$which is a conjugacy whenever this makes sense. The quasiconformal external conjugacy $H: \operatorname{closure}(A) \rightarrow$ closure $(\tilde{A})$ is thus constructed: $H(z)=H^{+}(z)$ iff $\Re(z) \geq 0$ and $H(z)=H^{-}(z)=$ $\widetilde{H^{+}(\bar{z})}$ iff $\Re(z) \leq 0$.

Step 3: In this step we construct a sequence of quasiconformal extensions of $H: \operatorname{closure}(A) \rightarrow \operatorname{closure}(\tilde{A})$.

\section{Fact 11.1}

$$
F^{-1}(E) \cup \operatorname{closure}\left(\cup_{i} \partial \Omega_{i} \cap \mathbb{R}\right)
$$

is closed for any closed subset $E$ of closure $(\Omega)$.

Proof: Let $x_{n} \in F^{-1}(E)$ and $x_{n} \rightarrow a$. If a subsequence of $\left\{x_{n}\right\}$ belongs to one component $\Omega_{j}$, then $F(a) \in E$, because $F$ : closure $\left(\Omega_{j}\right) \rightarrow \operatorname{closure}(\Omega)$ is continuous. Hence, $a \in F^{-1}(E)$ in this case. If there are infinitely many components $\Omega_{i}$ containing points of the sequence, then $a$ is the only limit point of such sequence of components because their diameters tend to zero. Hence, $a \in \operatorname{closure}\left(\cup_{i} \partial \Omega_{i} \cap \mathbb{R}\right)$.

For $n \geq 0$, let

$$
A_{n}=A \cup F^{-1} \cup \ldots \cup F^{-n}(A) \text { and } A_{\infty}=\cup_{n \geq 0} A_{n} .
$$




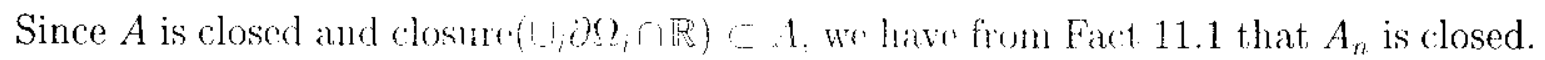
Moreover, let.

$$
U_{0}=\Omega\left(U_{1}=F \cdot{ }^{i}(\Omega)-U_{i} \Omega_{i} \ldots V_{n}=F^{-n}(\Omega)\right.
$$

Since $U_{1} \subset U_{0}$ this is a dremasing serpunuer of opren sels. These sets form the analogue of the Yoccoz puzzle pieces: thir compormenti of $J_{n}-F^{-n}(\Omega)$ are said to be the pieces of level $n$. It is easy to see that.

$$
A_{n+1} J I_{n+1}=\operatorname{losinc}(\Omega) \cdot n=0,1, \ldots
$$

One can extend $H: A \rightarrow A$ to a comjugary

$$
I / A_{\infty} \cdots, A_{x}
$$

see the following fact. Restrictions of $I /$ to $A_{n}$ even extend to a quasiconformal homeomorphism (but the dilatation of $\phi_{n}$ (an depend on $n$ ):

Fact 11.2 $H: A \rightarrow \tilde{A}$ can be entended:

1. For each $n=0.1, \ldots, H: A \rightarrow A$ crlorids to a conjugacy $H: A_{n} \rightarrow \tilde{A}_{n}$. (We shall also denote this homomomphism by $11_{1_{1} n_{1}}$.)

2. For every $n=0,1, \ldots$ there erists $\tilde{K}_{n}$ such that $H: A_{n} \rightarrow \tilde{A}_{n}$ extends to a $K_{n}$-quasiconformal homeomorphism is of 8 onto $[$.

Proof: Use the pullback construction (sce eg. [MS]). More precisely, given $n$ one can choose a $K_{n}$-quasiconfomal mapring $\phi: \Omega \rightarrow \Omega$ which is symmetric with respect to $\mathbb{R}$ such that $\phi_{\mid \partial A}=H$ and suld that $\phi\left(F^{\prime \prime}(c)\right)=F^{\prime}(c), r=1, \ldots, n+1$, where $c, \tilde{c}$ are the critical points of $F$ and $F$. Note that $K_{n}$ conld tend to infinity as $n \rightarrow \infty$.) Next set $\phi_{n}=H A_{n}$ on $A_{n}$ and define $\phi_{n}=F^{-n-1} \circ \phi \circ F^{n+1}$ on $U_{n+1}$ in such a way that $\phi_{n}$ becomes contimumes and so that $\bar{x}=\phi_{n}(x)$ means that $F^{k}(x) \in$ $\Omega_{i_{k}}$ iff $\tilde{F}^{k}(\tilde{x}) \in \tilde{\Omega}_{i_{k}}, k=1 \ldots \ldots$. (Continnity of $\phi_{n}$, detemines which preimage of

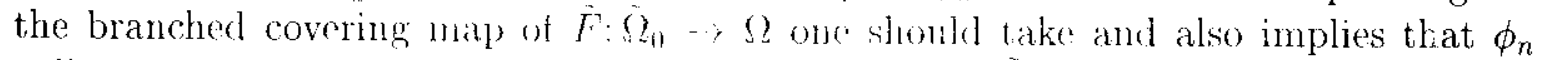
will be symmetric with mpece $10 \mathbb{E}$.) Then $\phi_{n}: \Omega \rightarrow \Omega$ is a homeomorphism which is $K$-quasiconformal on interior $\left.F{ }^{\prime}(A)\right) k=0 \ldots n$, and $K_{n}$-quasiconformal on $U_{n+1} . \phi_{n}: A_{n} \rightarrow \check{A}_{n}$ conjugates $F$ and $F$. len us consider the remaining set $L_{n}:=$ $\cup_{k=0}^{n} F^{-k}(\partial A)=\Omega \backslash\left(\cup_{k-1)}^{n}\right.$ interjor $\left.\left(F^{*}(A)\right) \cup C_{n+1}\right)$. Note that by Fact $11.1, L_{n}$ is

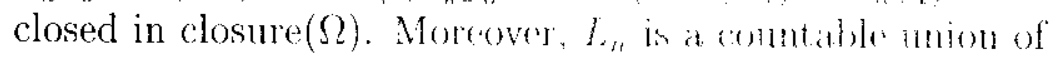

(a) rectifiable arcs: $\partial \Omega_{2}$ and components of $F^{-k}\left(\partial g_{j}\right)$

(b) subsets of components of $F$ k $(R \cap \Omega)$.

Therefore, $L_{n}$ is a closed subset of the plane with $\sigma$-finite linear measure, and, by [Stre], or see Theorem V.3.2 of [ 1 reVi]. $L_{n}$ is removable for any homeomorphism which is quasiconformal outside $L_{n \prime}$.

Since the dilatation $K_{n}$ can tend to infinity, wo nees to study the geometric structure of the sets $\Omega_{n}$ in more detail. To do this. if $x \in V_{n}$ let $\Omega^{n}(x)$ be the componont of $U_{n}$ containing this point (so $\Omega^{\prime \prime}(n)$ in the level $n$ pices which contains $x$ ). Note that 
for each $n>0$, there are infinitely many pieces of level $n$, and (what is worse) their 'diameters' are, in general, incomparable with each other. The following fact describes the geometry of these sets in more detail. Let $c$ be the critical point. For $n \geq 0$, let $\Omega^{n}(c)$ be the critical piece (i.e., the piece of level $n$ containing $c$ ). Then define the base point $c_{\Omega^{n}(c)}$ of this critical piece to be $c$. For any non-critical piece $\Omega^{n}$ define its base point $c_{\Omega^{n}}$ inductively so that $F\left(c_{\Omega^{n}}\right)=c_{F\left(\Omega^{n}\right)}$.

Denote by $\bmod (C)$ the modulus of an annulus $C$, by $\operatorname{diam}(I M)$ the Euclidean diameter of a planar set $M$, and by $d(M, N)$ the Euclidean distance between two planar sets $M, N$.

Fact 11.3 For each $n \geq 1$, there exist positive constants $M_{n}, R_{n}$ and $\epsilon_{n}, \epsilon_{n, n-1}$, such that the following properties hold.

1. Every level $n$ piece $\Omega^{n}$ has a simply connccted neighbourhood $W\left(\Omega^{n}\right)$ for which a) $\bmod \left(W\left(\Omega^{n}\right) \backslash \Omega^{n}\right) \geq M_{n}$,

b) F maps $W\left(\Omega^{n}\right)$ into $W\left(F\left(\Omega^{n}\right)\right.$ ) and restricted to $W\left(\Omega^{n}\right)$ is $\Omega^{n}$ is an (unbranched) covering onto its image,

c) $W\left(\Omega^{n}\right)$ is a subset of the unique level $(n-1)$ piece which contains $\Omega^{n}$,

d) $\Omega^{n}$ is roughly a disc:

$$
\sup _{z \in \partial \Omega^{n}}\left|z-c_{\Omega^{n}}\right| / \inf _{z \in \partial \Omega^{n}}\left|z-c_{\Omega^{n}}\right|<R_{n}
$$

2. a) for every piece $\Omega^{n}$ of level $n$ and every piece $\Omega^{n-1}$ of level $(n-1)$, if $\Omega^{n} \subset \Omega^{n-1}$, then $d\left(\Omega^{n}, \partial \Omega^{n-1}\right) / \operatorname{diam}\left(\Omega^{n}\right) \geq \epsilon_{n, n-1}$.

b) for every two different pieces $\Omega_{1}^{n}, \Omega_{2}^{n}$ of level $n$, if $\operatorname{diam}\left(\Omega_{1}^{n}\right) \geq \operatorname{diam}\left(\Omega_{2}^{n}\right)$, then $d\left(\Omega_{1}^{n}, \Omega_{2}^{n}\right) / \operatorname{diam}\left(\Omega_{2}^{n}\right) \geq \epsilon_{n}$.

Proof: Let us first prove assertions 1a)-1d) by induction on $n \geq 1$. Property (4) of Theorem A' implies these assertions hold for $n=1$. So assume 1a)-1d) hold for $1,2, \ldots, n-1$. Take a piece $\Omega^{n}$ of level $n$, and consider the piece $\Omega^{n-1}=F\left(\Omega^{n}\right)$ of level $(n-1)$. We need to show that $M_{n}$ and $R_{n}$ do not depend on $\Omega^{n}$ (but only on $n)$. If $W\left(\Omega^{n-1}\right)$ does not contain the critical value $c_{1}$, then define $W\left(\Omega^{n}\right)$ to be the preimage of $W\left(\Omega^{n-1}\right)$ by $F$ (remember that all branches of $F^{-1}$ are defined on a fixed neighbourhood of $\Omega$ ). Statements 1a)-1d) then hold; (for statement 1d) use the induction assumption and Koebe). Now consider the case that $c_{1} \in W\left(\Omega^{n-1}\right)$. Let $a>0$ be the distance between $c_{1}$ and $\partial \Omega^{n-1}\left(c_{1}\right)$. By the induction assumption $\left.1 \mathrm{~d}\right)$ for $n-1$, there are only finitely many pieces of level $(n-1)$ with diameter $\geq a$. Hence, one can choose corresponding neighbourhoods so that 1a)-1d) hold for these finitely many pieces.

If the diameter of $\Omega^{n-1}$ is smaller than $a$ and $\Omega^{n-1} \cap \Omega^{n-1}\left(c_{1}\right)=\emptyset$. then one can choose $M_{n-1}^{\prime} \leq M_{n-1}$ and a smaller neighbourhood $W^{\prime}$ of $\Omega^{n-1}$ inside $W\left(\Omega^{n-1}\right)$ such that $\bmod \left(W^{\prime} \backslash \Omega^{n-1}\right) \geq M_{n-1}^{\prime}$ and so that $\operatorname{diam}\left(W^{\prime}\right) \leq(3 / 2) a$. Then $c_{1} \notin W^{\prime}$ and $W\left(\Omega^{n}\right)$ can be taken as the diffeomorphic preimage of $W^{\prime}$. This proves 1a)-1c) for $n$ 
with $M_{n}=M_{n-1}^{\prime}$. From Koebe, induction and la)-1b) we get a constant $K_{n}$ in 1d) which does not depend on $\Omega^{n}$. If finally $\Omega^{n-1}=\Omega^{n \cdots 1}\left(c_{1}\right)$ then $\left.1 a\right)-1 c$ ) follows with $M_{n}=M_{n-1} / \ell$. Again 1d) follow'.

Statement 2a) is a direct corollary of the statements 1a) and 1d) we just pooved.

Let us now prove assertion $2 b)$ by induction. Let $n=1$. Let $\Omega_{i}, \Omega_{j}$ be two different level 1 pieces, and $W_{i}, W_{j}$ the corresponding neighbourhoods, see Theorem A (4). If $W_{i}, W_{j}$ are disjoint, then some uniform choice for $\epsilon_{1}>0$ exists because the distance between the pieces is comparable or larger than their diameters diameters. If $W_{i}, W_{j}$ are not disjoint, then $W_{j} \subset W_{i}$ (or $W_{i} \subset W_{j}$ if the diameter of the pieces is comparable), and again the distance between the pieces is larger than the distance between $\Omega_{j}$ and $\partial W_{j}$, which is larger than $\epsilon_{1} \cdot \operatorname{diam}\left(\Omega_{j}\right)$ where $\epsilon_{1}>0$ depends only on the lower bound for the $\bmod \left(W_{j} \backslash \Omega_{j}\right)$. (We always use that $\bmod \left(W_{j} \backslash \Omega_{j}\right)$ is universally bounded from below.) This proves $2 \mathrm{~b}$ ) for $n=1$. So assime $2 \mathrm{~b}$ ) hold for $n-1$. If $\Omega_{1}^{n}, \Omega_{2}^{n}$ are in different pieces of level $n-1$, then one can put $\epsilon_{n}=\epsilon_{n-1}$. if $\Omega_{1}^{n}, \Omega_{2}^{n} \subset \Omega^{n-1}$, then apply $F$ and use the induction assumption (one should consider two cases: $\Omega^{n-1}$ is critical or not).

Step 4: In this step we approximate the desired conjugacy by the $K$-quasiconformal mappings $H_{n}$ constructed below.

To do this, we need the following fact which uses that there are pieces $\Omega_{i(n)}$ of level 1 with $\operatorname{diam}\left(\Omega_{i(m)}\right) \rightarrow 0$ as $m \rightarrow \infty$ which intersects $\omega(c)$ (because $\omega(c)$ is non-minimal). This allows us to prove a statement similar to Proposition 3.3 of [LS1]:

Fact 11.4 There exist a sequence of integers $n_{m} \rightarrow \infty$, a neighbourhood $Q$ of the range $\Omega$, such that, for every $n_{m}$ :

(a) there exists a neighbourhood $Q_{n_{m}}$ of the critical piece $\Omega^{n_{n}}(c)$, such that $F^{n_{m}}$ maps $Q_{n_{m}}$ onto $Q$ in a $\ell$ to one fashion, and $F^{n_{m}}(c)$ lies in the central component $\Omega_{0}$ of the map $F$;

(b) if $x$ is so that $F^{j}(x) \in \Omega^{\prime \prime \prime}(c)$ with $j$ mimimal, then there exists a unix alent branch of $F^{-j}$ on $Q_{n_{m}}$ to a neighbouthood of $x$.

Proof: Pick such a small disc $\Omega_{i(m)}$, and let $k(m)$ be minimal so that $F^{k(m)}(c) \in \Omega_{i(m)}$. Note that $k(m) \rightarrow \infty$. Let $\Omega^{\prime}$ be a component of $F^{-k(m)}\left(\Omega_{n(m)}\right)$ containing $c$. Then $F^{k(m)}: \Omega^{\prime} \rightarrow \Omega_{i(m)}$ is a $\ell$ to one cover. Since $c$ is recurrent, there is a minimal $k^{\prime} \geq 1$ such that $F^{k(m)+k^{\prime}}(c)$ belongs to the domain $\Omega_{0}$. One can assume $k^{\prime} \geq 2$ (for infinitely many $k(m))$. Then we can define $n_{m}=h(m)+k^{\prime}$. The neighbourhood $Q$ of $\Omega$ exists because of Property 4 of 'Theorem A'. This completes the proof of Fact 11.4.

\section{Denote}

$$
\Omega_{0}(m)=\Omega^{n_{m}}(c)
$$

On the boundary of $\Omega_{0}(m)$ (and on part of its interior) $H$ is already defined by the previous pullback construction. We now modify the definition of $H$ on $\Omega_{0}(m)$ and also on all its preimages. We do this as follows. Choose a cuasiconformal homeomorphism $\hat{H}: \Omega \rightarrow \tilde{\Omega}$ which coincides with $H$ on the boundary of $\Omega$ and which maps $F^{n_{m}}(c)$ to 
$\tilde{F}^{n_{m}}(\tilde{c})$. Since $F^{n_{m}}(c)$ is roughly in the middle of $\Omega$ (and the same holds for the other map), one can choose $\hat{H}$ so that its quasiconformal dilatation is at most some universal number $K$. Now redefine the partially defined map $H$ to a map $H_{m}: \Omega_{0}(m) \rightarrow \tilde{\Omega}_{0}(m)$ so that $H_{m}=\tilde{F}^{-n_{m}} \circ \hat{H} \circ F^{n_{m}}$. Since $\hat{H}\left(F^{n_{m}}(c)\right)=\tilde{F}^{n_{m}}(\tilde{c}), H_{m}$ is well-defined and has quasiconformal dilatation $\leq K$. Next define $H_{m}$ from a preimage of $\Omega_{0}(m)$ to the corresponding preimage of $\tilde{\Omega}_{0}(m)$ as a conjugacy, but only consider those preimages $F^{-j}\left(\Omega_{0}(m)\right)$ whose forward iterates up to $j-1$ do not enter $\Omega_{0}(m)$. Outside all these preimages let $H_{m}$ coincide with the map $H$ constructed by the pullback construction in the previous section.

Fix $m$ and partition $\Omega$ into the following 4 sets:

$X_{m}(a)$ is the set of points $x$ such that there exists $n \geq 0$ so that $F^{n}(x)$ lies in the (open) central piece $\Omega_{0}(m)$;

$X_{m}(b)$ is the set of $x$ such that there exists a minimal $n \geq 0$ so triat $F^{n}(x)$ lies in the interior of the annulus $A$ and $F^{k}(x) \notin \Omega_{0}(m), k=0, \ldots, n-1$;

$X_{m}(c)$ is the set of $x$ such that there exists a minimal $n \geq 0$ so that $F^{n}(x)$ lies in the boundary of $A$ and $F^{k}(x) \notin \Omega_{0}(m), k=0, \ldots, n-1$;

Finally, $X_{m}(d)$ is the set of $x$ such that $F^{n}(x)$ is well-defined and lies outside $\Omega_{0}(m)$, for every $n=0,1,2, \ldots$.

Until now, $H_{m}$ has been defined on the sets $X_{m}(a), X_{m}(b), X_{m}(c)$. Extend $H_{m}$ naturally to the set $X_{m}(d)$ as follows. Any point $x \in X_{m}(d)$ is contained in a nested sequence of the puzzle pieces $\Omega^{n}(x), n>n_{m}$, such that each $\Omega^{n}(x)$ is mapped by $F^{n-n_{m}}$ univalently onto some non-critical piece of some fixed level $n_{m}$ (where $n_{m}$ does not depend on $x$ but only on $m$ ), and moreover, this map extends univalently onto one level up, i.e., from $\Omega^{n-1}(x)$ onto some piece of level $n_{m}-1$. Note that the distortion of this map does not depend on the point $x$ (but only on $m$ ) because the modulus of the annuli between $n_{m}-1$ and $n_{m}$ levels is bounded from below by some positive constant $m_{-}$(which is fixed if $m$ is fixed). All $n_{m}$-components are roughly discs with respect to their base points, see Fact 11.3(1d). We conclude that all $\Omega^{n}(x)$ are also roughly discs (with respect to their base points): the inequality of Fact 11.3(1d) holds for them but with a constant $R_{n}$ which only depends $m$ (i.e. it does not depend on $n$ and $x$ ). In particular, the (Euclidean) diameters of $\Omega^{n}(x)$ tend to zero as $n \rightarrow \infty$ uniformly for all $x \in X_{m}(d)$. For $x \in X_{m}(d)$ define $H_{m}(x)$ dynamically as the unique point $y \in \tilde{\Omega}$ with the same itinerary, i.e., for every $n \geq 0, F^{n}(x) \in \Omega_{i_{n}}$ if and only if $\tilde{F}^{n}(y) \in \tilde{\Omega}_{i_{n}}$. Thus we have that

$$
H_{m}: \Omega \rightarrow \tilde{\Omega}
$$

is well-defined homeomorphism, which is a conjugacy between $F$ and $\tilde{F}$ everywhere except in the central domain $\Omega_{0}(m)$. Observe that the sequence $\left\{H_{m}\right\}_{m>1}$ converges uniformly to a conjugacy $h$ between $F, \tilde{F}$.

So we now need to show that $h$ is quasiconformal. To this end, fix again $m$ and consider $H_{m}$ at different points. First of all, $H_{m}$ is $K$-quasiconformal on the open sets $X_{m}(a), X_{m}(b)$, where $K$ does not depend on $m$ (because $H_{m}$ is $K$-quasiconformal on $\Omega_{0}(m)$; on the interior of $A$ one has that $H_{m}=H$ is $K$-quasiconformal). Thus it is enough to prove the following three facts (where the dilatations of $H_{m}$ in the last two facts can depend on $m$ ). 
Fact $11.5 X_{m}(c), X_{m}(d)$ are of zero Lebesgue measure.

Fact 11.6 $H_{m}$ is quasiconformal at any point from $X_{m}(d)$.

Fact $11.7 H_{m}$ is quasiconformal at any point from $X_{m}(c)$.

By quasiconformality in the last two of these statements we mean that $\left(^{* * *}\right.$, below holds at any point from $X_{m}(c)$ and $X_{m}(d)$.

Let us first show that these threo facts imply Theorem B'. Note that these facts imply (cf. [GS2]) that the homeomorphism $H_{m}$ is $K$-quasiconformal everywhere (see e.g. Theorem V.3.3 of [LeVi]). On the other hand, the sequence $\left\{H_{m}\right\}_{m>1}$ converges uniformly to a conjugacy $h: \Omega \rightarrow \tilde{\Omega}$ between $F$ and $\tilde{F}$. Hence $h$ is quasiconformal on $\Omega$. So let us prove these facts.

Proof of Fact 11.5: That the Lebesgue measure of $X_{n}(c)$ is zero is trivial: $X_{m}(c)$ is equal to $\cup_{n \geq 0} F^{-n}(\partial A)$. Here $\partial A$ has Lebesgue measure zero since it is a countable union of rectifiable curves $\partial \Omega_{i}$ and a subset of the real axis.

So let us show by contradiction that $\left|X_{m}(d)\right|=0$. So let $y$ be a Lebesgue density point of $X_{m}(d)$. Consider the same nested sequence of pieces $\Omega^{n}(y), n>n_{m}$, as before. We know that the distortion of $F^{n-n_{m}}: \Omega^{n}(y) \rightarrow \Omega^{n_{m}}\left(y_{n}\right)$, where $y_{n}=F^{n-n_{m}}(y)$, is uniformly bounded (it only depends on $m$ ), and all $\Omega^{n}(y)$ are roughly discs. Therefore (since $X_{m}(d)$ is forward invariant under $\left.F\right)$ the density of $X_{m}(d)$ in $\Omega^{n}(y)$, i.e., $\mid \Omega^{n}(y) \cap$ $X_{m}(d)|/| \Omega^{n}(y) \mid$ and also $\left|\Omega^{n_{m}}\left(y_{n}\right) \cap X_{m}(d)\right| /\left|\Omega^{n_{m}}\left(y_{n}\right)\right|$ both tend to 1 as $n \rightarrow \infty$. On the other hand, each piece $\Omega^{n_{m}}\left(y_{n}\right)$ of level $n_{m}$ is mapped by an $F$-iterate for the first time either to a central piece (of a level $<n_{m}$ ) or to $\Omega$, and the distortion of this map is uniformly bounded because $n_{m}$ is fixed. Therefore, there exists a fixed piece $Q$ which is either a critical piece of level $<n_{m}$ or which is equal to $\Omega$ so that the density of the set $X_{m}(d)$ in $Q$ is equal to one. In particular, $X_{m}(d) \cap \Omega_{0}(m) \neq 0$, a contradiction. This completes the proof of Fact 11.5.

Proof of Fact 11.6: To prove that $H_{m}$ is quasiconformal we are going to use the following recent surprising result by Heinonen-Koskela, see [HK].

${ }^{* * *}$ ) A homeomorphism $\varphi$ of the plane (more general, of $\mathbb{R}^{d}$ ) is quasiconformal, if there exists $L$ such that for any point $x$ there exists a sequence of radii $r_{n} \rightarrow 0$, such that, for any $n$,

$$
\sup _{|y-x|=r_{n}}|\varphi(y)-\varphi(x)| / \inf _{|y-x|=r_{n}}|\varphi(y)-\varphi(x)|<L .
$$

So it makes sense to all $\varphi$ quasiconformal at a point $x$ if $\left(^{* * *}\right)$ holds.

In our case we shall take $\varphi=H_{m}$ and $x \in X_{m}(c) \cup X_{m}(d)$. Note that we do not mind if $L$ from $(* * *)$ does depend on $m$. (In fact, for points in $X_{m}(c)$ we can take any sequence $r \rightarrow 0$.) In order to prove $\left({ }^{* *}\right)$, we will use that $H_{m}$ is strongly related to quasiconformal extensions $\phi_{n}$ of $H: A_{n} \rightarrow A_{n}$ when $n$ is large. 
So fix $m$ and consider a point $x \in X_{m}(d)$. Our aim is to show that $\left(^{* * *}\right)$ is satisfied when we take $r_{n}$ equal to $2 d_{n}(x)$, where $d_{n}(x)$ is the diameter of $\Omega^{n}(x)$. (The factor 2 is because we need space between $\Omega^{n}(x)$ and the circle $\left\{y:|y-x|=2 d_{n}\right\}$, since $x$ can be close to the boundary of $\Omega^{n}(x)$.)

Note that all 'universal' constants below as well as statements on the shapes of discs can depend on $m$ but these constants will not depend on $x$.

As before, there exists $m_{-}=m_{-}(m)>0$ so that for each $n \geq 0$ and each $x \in X_{m}(d)$, the modulus of the annulus $\Omega^{n-1}(x) \backslash \Omega^{n}(x)$ is bounded from below by $m_{-}$. (These moduli may not be bounded from above.) Hence there exists $j$ (which only depends on $m$ ), such that for any $n$, the modulus of the annulus $\Omega^{n-j}(x) \backslash \Omega^{n}(x)$ is so big that the Euclidean disc $B_{n}:=B\left(x, 2 d_{n}(x)\right)$ centered at $x$ with radius equal to $2 d_{n}(x)$ is contained in $\Omega^{n-j}(x)$ (and certainly contains $\Omega^{n}(x)$ ). This is because $\bmod \left(B_{n} \backslash \Omega^{n}(x)\right.$ ) lies between two universal positive numbers.

$F^{n-j-n_{m}}$ maps $B_{n}$ to a set between pieces of levels $n_{m}+j$ and $n_{m}$. Note that these iterates of $B_{n}$ do not intersect the critical piece $\Omega_{0}(m)$, because the latter piece has level $n_{m}$. Since the map $F^{n-j-n_{m}}$ has universally bounded distortion on $\Omega^{n-j}(x)$, the image $B^{\prime}=F^{n-j-n_{m}}\left(B_{n}\right)$ is roughly a Euclidean disc centered at

$$
x^{\prime}=F^{n-j-n_{m}}(x),
$$

is contained in a piece of level $n_{m}$, and its diameter is comparable with the diameter of

$$
P:=\Omega^{n_{m}+j}\left(x^{\prime}\right)
$$

One can take everything back by the corresponding branch of $\tilde{F}^{-\left(n-j-n_{m}\right)}$ with bounded distortion to $\tilde{x}=H_{m}(x)$, and hence $\left(^{* * *}\right)$ holds for $x \in X_{m}(d)$ (and therefore Fact 11.6 is proved) provided we can prove the following

Claim 1: For each set $B^{\prime}$ as above, the image $\tilde{B}^{\prime}=H_{m}\left(B^{\prime}\right)$ is roughly a disc centered at $\tilde{x}=H_{m}\left(x^{\prime}\right)$.

Proof of Claim 1: Note that

$$
P=\Omega^{n_{m}+j}\left(x^{\prime}\right) \subset B^{\prime} \subset \Omega^{n_{m}}\left(x^{\prime}\right) .
$$

The levels of the two puzzle pieces only depends on $m$, but since their diameters can be incomparable, this does not help much. The idea of the proof of the claim is to compare the set $B^{\prime}$ with a larger set which has dynamical meaning by adding to $B^{\prime}$ certain puzzle pieces. Thus $H_{m}\left(B^{\prime}\right)$ is contained in a set whose boundary is the image by some map $\phi_{k}$ (the quasiconformal extension of the pullback map $H: A_{\infty} \rightarrow \tilde{A}_{\infty}$ from Step 3). The quasiconformality of $\phi_{k}$ will then allow us to conclude the claim.

Let us start by noting that $B^{\prime}$ is roughly a disc around $x^{\prime}$ and $\bmod \left(B^{\prime} \backslash P\right)$ is universally bounded from above and from zero (as before universal at least for a given choice of $m$ ). Hence, there exists universal $r>r^{\prime}>0$ such that

$$
r^{\prime} \cdot \operatorname{diam}(P) \leq d(y, P) \leq r \cdot \operatorname{diam}(P)
$$

for every $y \in \partial B^{\prime}$. 
Fix a point $y \in \partial B^{\prime}$, and consider its iterates up to $F^{j}(y)$. The following three cases can occur:

1. There exists a minimal $i_{0}, 0 \leq i_{0} \leq j$, such that $F^{i_{0}}(y) \in A_{n_{m}-1}$ and $F^{i}(y) \notin$ $\Omega_{0}(m):=\Omega^{n_{m}}(c)$ for $0 \leq i \leq i_{0}$ (as remarked before $A_{n_{m-1}}=\cup_{k=0}^{n_{m}-1} F^{-k}(A)$ is the complement to the pieces of level $n_{m}$, and $\Omega_{0}(m)=\Omega^{n_{m}}(c)$ is the critical piece of the level $n_{m}$ ). One can write:

$$
H_{m}(y)=\left.\tilde{F}^{-i_{0}} \circ H\right|_{{G_{n_{m}-1}}} \circ F^{i_{0}}(y)=\left.H\right|_{A_{n_{m}-1+i_{0}}}(y)=\phi_{n_{m}-1+i_{0}}(y)=\phi_{n_{m}+j+1}(y)
$$

where $\phi_{n}$ is the quasiconformal extension of $\left.H\right|_{A_{n}}$ to $\Omega$ constructed in Fact 11.2. In this case define $Q(y)=\emptyset$.

2. For each $i$ with $0 \leq i \leq j$ the iterate $F^{i}(y)$ is well-defined, $F^{j}(y) \in U_{n_{n}}$ (i.e., lies in $\Omega^{n_{m}}\left(F^{j}(y)\right)$ ), and $F^{i}(y) \notin \Omega_{0}(m)$ for $0 \leq i \leq j$. Then $y \in Q^{\prime}(y):=\Omega^{n_{m}+j}(y)$. Note that $Q^{\prime}(y) \cap P=\emptyset$ because otherwise two pieces of the same level $n_{m}+j$ coincide which is impossible because $P$ does not intersect $\partial B^{\prime}$. Next let us look at one level down and consider two subcases.

(a) $y$ is ouside the pieces of level $n_{m}+j+1$. Then $F^{j+1}(y)$ is outside the pieces of level $n_{m}$, and

Then define $Q(y)=\emptyset$.

$$
H_{m}(y)=\phi_{n_{m}+j+1}(y)
$$

(b) $y$ is inside a piece $\Omega^{n_{m}+j+1}(y)$ of level $\left(n_{m}+j+1\right)$. Then define $Q(y):=$ $\Omega^{n_{m}+j+1}(y)$. Note that $H_{m}(y) \in \tilde{\Omega}^{n_{m}+j+1}$, where the latter is the piece of $\tilde{F}$ of level $\left(n_{m}+j+1\right)$ for which $\partial \tilde{\Omega}^{n_{m}+j+1}=\left.H\right|_{A_{n_{m}+j+1}}(\partial Q(y))$. In other words,

$$
H_{m}(y) \in \phi_{n_{m}+j+1}(Q(y)) \text {. }
$$

It is useful to note at this point that the sets $F^{i}(P), 0 \leq i \leq j$, do not intersect $\Omega_{0}(m)$ (because the iterates of $x$ do not meet $\Omega_{0}(m)$ ). Therefore, as above,

$$
H_{m}\left(x^{\prime}\right) \in H_{m}(P)=\phi_{n_{m}+j}(P)=\phi_{n_{m}+j+1}(P)
$$

where $x^{\prime}=F^{n-j-n_{m}}(x)$ as before.

3. There exists a minimal $i_{0}, 0 \leq i_{0} \leq j$, such that $F^{i_{0}}(y) \in \Omega_{0}(m)$. Then $y$ lies in $\Omega^{n_{m}+i_{0}}(y)$, which is a preimage of the critical piece $\Omega_{0}(m)$ by a branch of $F^{-i_{0}}$. Consider again two subcases.

(a) $\operatorname{diam}\left(\Omega^{n_{m}+i_{0}}(y)\right) \leq(1 / 10) \cdot r^{\prime} \cdot \operatorname{diam}(P)$. Then define $Q(y)=\Omega^{n_{m}+i_{0}}(y)$. Note that $H_{m}(y) \in \tilde{\Omega}^{n_{m}+i_{0}}$, where $\partial \tilde{\Omega}^{n_{m}+i_{0}}=\left.H\right|_{A_{n_{m}+i_{0}}}(\partial Q(y))=\left.H\right|_{A_{n_{m}+j+1}}(\partial Q(y))$. In
other words, again

$$
H_{m}(y) \in \phi_{n_{m}+j+1}(Q(y)) \text {. }
$$

(b) If $\operatorname{diam}\left(\Omega^{n_{m}+i_{0}}(y)\right)>(1 / 10) \cdot r^{\prime} \cdot \operatorname{diam}(P)$, then define $Q(y)=\emptyset$. By construction $H_{m}$ is $K$-quasiconformal on $\Omega^{n_{m}+i_{0}}(y)$, and $\phi_{n_{m}+j+1}=H_{m}$ on the boundary of $\Omega^{n_{m}+i_{0}}(y)$; Moreover, there are at most finitely many $\Omega^{n_{m}+i_{0}}(y)$ as in this subcase, see Fact 11.3(1d) and Fact 11.4. In addition, $\Omega^{n_{m}+i_{0}}(y) \cap P=\emptyset$ because otherwise the level $n_{m}+j$ piece $P$ is contained in the level $n_{m}+i_{0}$ piece $\Omega^{n_{m}+i_{0}}(y)$ and then $F^{i_{0}}\left(x^{\prime}\right) \in F^{i_{0}}(P) \subset F^{i_{0}}\left(\Omega^{n_{m}+i_{0}}(y)\right)=\Omega_{0}(m)$, a contradiction. 
Now define a new map $\phi^{\prime}$. Let $\phi^{\prime}$ be equal to $\phi_{n_{m}+j+1}$ everywhere in $\Omega$ except for pieces $\Omega^{n_{m}+i_{0}}(y)$ as in 3(b), on which we define $\phi=H_{m}$. Define also

$$
C^{\prime}=\partial B^{\prime} \cup\left\{Q(y) ; y \in \partial B^{\prime}\right\} .
$$

We have proved that

$$
H_{m}\left(\partial B^{\prime}\right) \subset \phi^{\prime}\left(C^{\prime}\right)
$$

and

$$
H_{m}\left(x^{\prime}\right) \in \phi^{\prime}(P)
$$

Observe that $\phi^{\prime}$ is a homeomorphism of $\Omega$ onto $\tilde{\Omega}$, which is $K_{n_{m}+j+1}$-quasiconformal on any open set $U$ for which $\phi^{\prime} \mid U=\phi_{n_{m}+j+1}$ and $K$-quasiconformal when $\phi^{\prime} \mid U=H_{m}$. Hence, $\phi^{\prime}$ is $K_{n_{m}+j+1}$-quasiconformal everywhere except along finitely many piecewise analytic curves (the boundaries of the pieces $\Omega^{n_{m}+i_{0}}(y)$ as in Case $\left.3 b_{1}\right)$. Therefore, $\phi^{\prime}$ is $K^{\prime}$-quasiconformal on $\Omega$, where $K^{\prime}=K_{n_{m}+j+1}$ depends only on $m$. In order to use this, we need to show that $C^{\prime}$ has a good geometric structure:

Claim 2: There exist two universal constants $0<b<a$, such that, for every $z \in C^{\prime}$,

$$
b \cdot \operatorname{diam}(P) \leq d(z, P) \leq a \cdot \operatorname{diam}(P) .
$$

Before we prove Claim 2, let us show that it implies Claim 1. Indeed, take $x^{\prime \prime}=$ $\left(\phi^{\prime}\right)^{-1} \circ H_{m}\left(x^{\prime}\right) \in P$. Assuming claim 2 is true, for every $z \in C^{\prime}$,

$$
b \cdot \operatorname{diam}(P) \leq d\left(z, x^{\prime \prime}\right) \leq(1+a) \cdot \operatorname{diam}(P) .
$$

Hence, $H_{m}\left(\partial B^{\prime}\right)$ surrounds the set $\Gamma_{1}:=\phi^{\prime}\left(B\left(x^{\prime \prime}, b \cdot \operatorname{diam}(P)\right)\right)$ and is contained in the set $\Gamma_{2}:=\phi^{\prime}\left(B\left(x^{\prime \prime},(1+a) \cdot \operatorname{diam}(P)\right)\right)$, where $B(z, r)=\{y ;|y-z| \leq r\}$. Let $\tilde{x}=H_{m}\left(x^{\prime}\right)=\phi^{\prime}\left(x^{\prime \prime}\right)$. Since $\phi^{\prime}$ is $K^{\prime}$-quasiconformal, there exists $D=D\left(K^{\prime}\right)$, such that

$$
B\left(\tilde{x}, \tilde{r}_{1}\right) \subset \Gamma_{1} \subset B\left(\tilde{x}, D \cdot \tilde{r}_{1}\right)
$$

where $\tilde{r}_{1}=\min \left\{|\tilde{x}-y| ; y \in \Gamma_{1}\right\}$, and

$$
B\left(\tilde{x}, \tilde{r}_{2} / D\right) \subset \Gamma_{2} \subset B\left(\tilde{x}, \tilde{r}_{2}\right),
$$

where $\tilde{r}_{2}=\max \left\{|\tilde{x}-y| ; y \in \Gamma_{2}\right\}$. Hence

$$
\bmod \left(\Gamma_{2} \backslash \Gamma_{1}\right) \geq 2 \pi \cdot \log \left(\frac{\tilde{r}_{2} / D}{D \tilde{r}_{1}}\right)
$$

We need to prove that $\tilde{r}_{2} / \tilde{r}_{1}$ is universally bounded from above. But since $\phi^{\prime}$ is $K^{\prime}$ quasiconformal and $\left(\Gamma_{2} \backslash \Gamma_{1}\right)$ is the $\phi^{\prime}$ image of the annulus $\left\{z ; b \cdot \operatorname{diam}(P) \leq\left|z-x^{\prime \prime}\right| \leq\right.$ $(1+a) \cdot \operatorname{diam}(P)\}$, we get

$$
\left(2 \pi / K^{\prime}\right) \cdot \log ((1+a) / b) \leq \bmod \left(\Gamma_{2} \backslash \Gamma_{1}\right) \leq\left(2 \pi \cdot K^{\prime}\right) \cdot \log ((1+a) / b) .
$$

This and the above inequality for $\log \left(\Gamma_{2} \backslash \Gamma_{1}\right)$ imply that the ratio $\tilde{r}_{2} / \tilde{r}_{1}$ cannot be larger than $D^{2} \cdot[(1+a) / b]^{K^{\prime}}$. So let us prove Claim 2. First of all, it holds for any 
$z \in \partial B^{\prime}$ : take $b=r^{\prime}$ and $a=r$. It also holds for any $z \in Q(y)$ for any $Q(y)$ (as above) for which $\operatorname{diam}(\Omega(y)) \leq(1 / 10) \cdot r^{\prime} \cdot \operatorname{diam}(P)$. This is because in that case it is enough to put $b=9 / 10 \cdot r^{\prime}$ and $a=r+1 / 10 \cdot r^{\prime}$. In particular it holds for all $z \in Q(y)$ with $Q(y)$ as in case $3(\mathrm{a})$.

So it remains to consider $z \in Q(y)$ where $Q(y)$ is as in $2(\mathrm{~b})$. To prove the righthand side inequality of claim 2 , we need to show that any such level $n_{m}+j+1$ piece $Q(y), y \in \partial B^{\prime}$, is not too large compared to $\operatorname{diam}(P)$. Indeed, for the level $n_{m}+j$ piece $Q^{\prime}(y)$ defined in case 2, we have $Q^{\prime}(y) \cap P=\emptyset$ and, by Fact, $11.3(2 \mathrm{a})$,

$$
\epsilon_{*} \cdot \operatorname{diam}(Q(y)) \leq d\left(Q(y), \partial Q^{\prime}(y)\right) \leq d(Q(y), P) \leq d(y, P) \leq r \cdot \operatorname{diam}(P),
$$

where $\epsilon_{*}=\epsilon_{n_{m}+j+1, n_{m}+j}$. Hence

$$
\operatorname{diam}(Q(y)) \leq\left(r / \epsilon_{*}\right) \cdot \operatorname{diam}(P)
$$

which prove the right-hand inequality of Claim 2. Let us now prove the left-hand side inequality. Since we consider $Q(y)$ such that $\operatorname{diam}(Q(y)) \geq(1 / 10) \cdot r^{\prime} \cdot \operatorname{diam}(P)$, we get by Fact $11.3(2 b)$ :

$$
\begin{aligned}
d(Q(y), P) & \geq d\left(Q^{\prime}, P\right) \geq \epsilon_{n_{m}+j} \cdot \min \left\{\operatorname{diam}\left(Q^{\prime}\right), \operatorname{diam}(P)\right\} \geq \\
& \geq \epsilon_{n_{m}+j} \cdot \min \{\operatorname{diam}(Q), \operatorname{diam}(P)\} \geq \epsilon_{n_{m}+j} \cdot \min \left(1, r^{\prime} / 10\right) \cdot \operatorname{diam}(P) .
\end{aligned}
$$

This proves that one can also choose $b>0$ uniformly in the left-hand side inequality of Claim 2. This completes the proof of claim 2 and therefore also the proof of claim 1 .

Proof of Fact 11.7: Now consider $H_{m}$ at points $x \in X_{m}(c)$. By definition, for some $n \geq 0, \quad y:=F^{n}(x)$ lies in the inner boundary of $A$, i.e. in the closure of the union of $\partial \Omega_{i}$, and $F^{k}(x) \notin \Omega_{0}(m), k=0, \ldots, n$. There are two substantially different cases:

(a) $y$ is not real, belongs to the boundary of some $\Omega_{j}$, and $F(y)$ is not real as well,

(b) either $y \in \partial A \cap \mathbb{R}$ or $y \in \Omega^{1}(c)$ and $F(y)$ is real (i.e. one of the end points of $\Omega \cap \mathbb{R})$.

In turn, for both (a) and (b), there are two subcases:

(1) there exists $k, 0 \leq k \leq n$, such that $F^{k}(x) \in \partial \Omega_{0}(m)$

(2) $F^{k}(x) \notin \Omega_{0}(m)$, for all $k=0, \ldots, n$.

In the cases (a1)-(a2) there exists a neighbourhood $U$ of $z=F^{k}(x)$ in case (a1) and of $z=y$ in case (a2), such that $U \subset A_{n-k}$ in (a1) and $U \subset A_{1}$ in (a2) (remember that $A_{k}=\cup_{i=0}^{k} F^{-i}(A)$ ). Hence, the homeomorphism $H_{m}$ is quasiconformal at each point of $U$ except for a piecewise analytic arc (which is either $U \cap \Omega_{0}(m)$ or $U \cap \Omega_{j}$ ). Since such arcs are removable, $H_{m}$ is quasiconformal at $z \in U$

In case (b), the subcases (1)-(2) are very similar. Let us first consider the most difficult case (b2) assuming also that $y \in \partial A \cap \mathbb{R}$ (the second possibility that $y \in \Omega^{1}(c)$ with $F(y)$ real is easier). Since $F^{k}(x) \notin \Omega_{0}(m)$ for $k \leq n$, we have $H_{m}=\tilde{F}^{-n} \circ H_{m} \circ F^{n}$ in a small neighbourhood of the point $x$ (the size of the neighbourhood depends on $n$ ). Hence, it is enough to show that $H_{m}$ is quasiconformal at $y \in \partial A \cap \mathbb{R}$. In particular, $y$ 
is not inside any $\Omega_{i}$ but can be a point of $\partial \Omega_{i} \cap \mathbb{R}$. Take a round disc $B=B(y, r)$ with the center at $y$ and a small radius $r$. Similar to the proof of Claim 1 above, consider

$$
C=\partial B \cup\left\{\Omega^{2} ; \Omega^{2} \cap \partial B \neq \emptyset\right\},
$$

where $\Omega^{2}$ are the level 2 pieces. We have: $H_{m}(y)=\phi_{2}(y)$ and $H_{m}(\partial B) \subset \phi_{2}(C)$. Since $\phi_{2}: \Omega \rightarrow \tilde{\Omega}$ is quasiconformal, it is enough to prove that $C$ is roughly a. 'circle' around $y$, i.e. that there exist $r_{2}>r_{1}>0$ such that $C \cap\left\{z ;|z-y|=r_{i}\right\}=\emptyset, i=1,2$ and such that $r_{2} / r_{1}$ is universally bounded from above. To prove this, let $\Omega^{2} \cap \partial B \neq \emptyset$, and let $\Omega^{1}$ be the level 1 piece containing $\Omega^{2}$. Note that $y \notin \Omega^{1}$. As it follows from Fact $11.3(1 \mathrm{c})$, for example, there exists a universal $\epsilon_{m}>0$, such that

$$
d\left(\Omega^{2}, \partial \Omega^{1}\right) \geq \epsilon_{m} \cdot \operatorname{diam}\left(\Omega^{2}\right) .
$$

This implies that $\operatorname{diam}\left(\Omega^{2}\right) \leq r / \epsilon_{m}$ because $d\left(\Omega^{2}, \partial \Omega^{1}\right)>\epsilon_{m} \cdot\left(r / \epsilon_{m}\right)=r$ and since $y \notin \Omega^{1}$ this would imply that $\left(\Omega^{2}, y\right)>r$, a contradiction. Similarly, $d:=d\left(\Omega^{2}, y\right) \geq$ $r \cdot \epsilon_{m} /\left(1+\epsilon_{m}\right)$ because otherwise we would have: $d=d\left(\Omega^{2}, y\right) \geq d\left(\Omega^{2}, \partial \Omega^{1}\right) \geq \epsilon$. $\operatorname{diam}\left(\Omega^{2}\right) \geq \epsilon \cdot(r-d)>r \cdot \epsilon /(1+\epsilon)$, i.e. a contradiction. Thus one can take $r_{1}=r \cdot \epsilon /(1+\epsilon)$ and $r_{2}=r+r / \epsilon=r \cdot(1+\epsilon) / \epsilon$.

If $y$ is as in case $\mathrm{b}(1)$ then $H_{m}=\tilde{F}^{-k} \circ H_{m} \circ F^{k}$ and so it is enough to show that $\left.{ }^{(* *}\right)$ holds for $H_{m}$ near $z=F^{k}(y) \in \partial \Omega_{0}(m)$. Using again a set $C$ as above, $\left.{ }^{* * *}\right)$ again follows at $z$.

Thus we have proved that $\left(^{* * *}\right)$ holds at any point of $X_{m}(c)$ and befor we proved $\left({ }^{* *}\right)$ for any point of $X_{m}(d)$ with a special choice of $r_{n} \rightarrow 0$. The proof of Theorem $\mathrm{B}^{\prime}$ is completed.

Remark. Combining the considerations used in the cases $x \in X_{m}(d)$ and $x \in X_{m}(c)$, one can show that $\left(^{* * *}\right)$ holds at $x \in X_{m}(d)$ for an arbitrary small enough $r_{n}$, i.e. show that $H_{m}$ is quasiconformal without the use of the deep result of [HK].

\section{Proof of Theorem C: Density of Axiom A maps in families}

Let $g_{\lambda}: \cup_{i=0}^{i_{0}} I^{i}(\lambda) \rightarrow I$ be a family of maps of the class AC, where $\lambda \in \Lambda:=\left[\Lambda_{1}, \Lambda_{2}\right]$. Denote $c_{\lambda} \in I^{0}(\lambda)$ the critical point of $g_{\lambda}$, and $\omega_{\lambda}(c)$ the omega-limit set of $c(\lambda)$ by $g_{\lambda}$. Assume that:

a) the itineraries of the critical point of $g_{\Lambda_{1}}$ and $g_{\Lambda_{1}}$ are different;

b) $g_{\lambda}(x)$ is real analytic on $(\lambda, x)$ in a neighbourhood of every point $\left(\lambda_{0}, x_{0}\right)$, where $\lambda_{0} \in\left(\Lambda_{1}, \Lambda_{2}\right)$ and $x_{0} \in \operatorname{closure}\left(\cup_{i=0}^{i_{0}} I^{i}\left(\lambda_{0}\right)\right)$;

c) there exists $\lambda \in\left(\Lambda_{1}, \Lambda_{2}\right)$ such that $g_{\lambda}$ has no parabolic periodic orbits.

Let $A=\left\{\lambda \in \Lambda: g_{\lambda}\right.$ is Axiom $A$ map $\}$ and $S$ be its complement. Given $\lambda_{0} \in S$, let $S\left(\lambda_{0}\right)$ be connected component of $S$ containing $\lambda_{0}$. In this section, we address the important question whether the set $A$ is dense (or equivalently, $S(\lambda)=\{\lambda\}$ for every $\lambda \in S)$. 
Observe that if $g_{\lambda}(x)=g_{0}(x)+\lambda$, then $A$ is dense in this family, because all the branches "move up" with $\lambda$ (see Section 8 and also Lemmas 12.2-12.3 below). In particular, Axiom A maps are dense in the family $x \mapsto k x+\lambda-k / 2 \pi \sin (2 \pi x), \lambda \in \mathbb{R}$, of Arnold's maps, and in the family of Blaschke products of Example 2 of the Introduction. For the same reason, Axiom A maps are dense in the space of all maps $g \in \mathbf{A C}$.

Let us consider this problem for a family $g_{\lambda}$ satisfying the conditions a)-c) from the beginning of this section. It is very easy to see that $S\left(\lambda_{0}\right)=\left\{\lambda_{0}\right\}$ if $c$ is attracted to an attracting or parabolic periodic orbit of $g_{\lambda_{0}}$ (see Lemma 12.2) or eventually periodic (use condition a) from above and Lemma 12.3).

The cases when $\omega(c)$ is infinite are much more difficult to consider. We use Theorems A, B, A', B', and a method of [Ko, Sect. 7.2, p. 69-70] to prove the following slight generalization of Theorem $\mathrm{C}$ :

Theorem C'. Assume $\omega_{\lambda_{0}}(c)$ is infinite.

C. If $\omega_{\lambda_{0}}(c)$ is minimal, then $S\left(\lambda_{0}\right)=\left\{\lambda_{0}\right\}$, i.e., $g_{\lambda_{0}}$ is approximated by Axiom $A$ maps $g_{\lambda}$.

$C^{\prime}$. If $\omega_{\lambda_{0}}(c)$ is not minimal, and the following condition $\left(^{*}\right)$ holds:

$\left(^{*}\right) \omega_{\lambda}(c)$ does not contain parabolic periodic orbits, where $\lambda$ is at least one end point of $S\left(\lambda_{0}\right)$,

then again $S\left(\lambda_{0}\right)=\{\lambda\}$.

For more information see Lemmas 12.4-12.5.

In particular, we have

Corollary 12.1. Let $P_{\lambda}$ be a holomorphic family of polynomials of Example 1 from the introduction. Then either all polynomials $P_{\lambda}$ are conformally conjugate or Axiom $A$ maps are dense in this family.

The proof of Theorem C' is contained in the following Lemmas. Let us start by fixing a closed subinterval $\left[e_{1}, e_{2}\right]$ of $\left(\Lambda_{1}, \Lambda_{2}\right)$.

Lemma 12.1. For every $n \geq 1$, the set $N_{n}=\left\{\lambda \in\left[e_{1}, e_{2}\right]: g_{\lambda}^{n}\right.$ has a parabolic fixed point $\}$ is finite. In particular, the set $N=\cup_{n \geq 1} N_{n}$ is at most countable. [MS].)

Proof: Use b) and c). (Actually we have much more, see Theorem IV.B' in

Lemma 12.2. Fix arbitrary $\lambda_{0}=0 \in\left(e_{1}, e_{2}\right)$. If $g_{0}$ has an attracting or parabolic periodic orbit $P_{0}$, which attracts $c_{0}$, then there is $\lambda$ arbitrary close to $\lambda_{0}$ from either side of $\lambda_{0}$, such that $g_{\lambda}$ is Axiom $A$ map.

Proof: Consider several cases.

1). $P_{0}$ is attracting. Then for every $\lambda$ close enough to $\lambda_{0}, g_{\lambda}$ has an attracting periodic orbit $P_{\lambda}$, which changes continuously with $\lambda$, tends to $P_{0}$ as $\lambda \rightarrow \lambda_{0}$, and attracts $c_{\lambda}$. By Lemma 12.1, one can choose $\lambda$ arbitrary close to $\lambda_{0}$, such that $g_{\lambda}$ has 
no parabolic periodic orbits. Then, by Mane's theorem [MS, p. 222-223], this $g_{\lambda}$ is Axiom A.

2). $P_{0}$ is a two-sided attracted parabolic periodic orbit (like 0 for $x-x^{3}$ ). Then, for any $\lambda$ close enough to $\lambda_{0}$, there exists an attracting periodic orbit (close to $P_{0}$ ) which attracts $c_{\lambda}$. For this $\lambda$, we apply 1 ).

3). $P_{0}$ is a one-sided attracted parabolic periodic orbit (like 0 for $x+x^{2}$ ). There are two subcases:

$3 a)$. For $\lambda$ arbitrary close to $\lambda_{0}, g_{\lambda}$ has a nearby to $P_{0}$ periodic crbit(s). Then one of these orbits is attracting, and it attracts the critical point. Then we apply 1).

$3 \mathrm{~b})$. If $3 \mathrm{a}$ ) does not hold, then we proceed as in [Le1]. Let $p$ be the period of $P_{0}$. Then $g_{0}^{p i}\left(c_{0}\right) \rightarrow a_{0}$ as $i \rightarrow+\infty$, where $a_{0} \in P_{0}$. Moreover, since $P_{0}$ is the one-sided attracted orbit, one can assume that $g_{0}^{p i}\left(c_{0}\right)<a_{0}, i=1,2, \ldots$. On the other hand, since there are no wandering intervals, there exists a sequence $g_{0}^{-n_{i}}\left(c_{0}\right)$ of preimages of $c_{0}$, which tends to $a_{0}$ from the right side. By continuity, for every small $\epsilon>0$ and for every $i_{0}$, there exists $\delta>0$, such that for every $\lambda^{\prime} \in\left(\lambda_{0}-\delta, \lambda_{0}+\delta\right)$,

$$
g_{\lambda^{\prime}}^{-n_{i_{0}}}\left(c_{\lambda^{\prime}}\right) \in\left(a_{0}-\epsilon, a_{0}+\epsilon\right)
$$

But since there are no fixed points of $g_{\lambda}^{p}$ in a neighbourhood of $a_{0}$, if $\lambda \neq \lambda_{0}$ is close to $\lambda_{0}$, there exists (for the above $\lambda^{\prime}$ ) $i_{1}$, such that

$$
g_{\lambda^{\prime}}^{p i_{1}}\left(c_{\lambda^{\prime}}\right)>a_{0}+\epsilon
$$

Changing now $\lambda$ from $\lambda_{0}$ to $\lambda^{\prime}$, we must meet $\lambda$ such that

$$
g_{\lambda}^{-n_{i_{0}}}\left(c_{\lambda}\right)=g_{\lambda}^{p i_{1}}\left(c_{\lambda}\right)
$$

Therefore $c_{\lambda}$ is periodic, and we can again apply 1 ).

Lemma 12.3. If the itinerary of $c_{\lambda}$ is not constant for any $\lambda$ in a neighbourhood of $\lambda_{0}$, then there exists $\lambda$ arbitrary close to $\lambda_{0}$, such that $c_{\lambda}$ is attracted by an attracting or neutral periodic orbit of $g_{\lambda}$.

Proof: By the continuity of the itinerary on $\lambda$, one can choose $\lambda$ arbitrary close to $\lambda_{0}$, so that the itinerary is periodic. It implies $g_{\lambda}$ has a periodic interval containing the critical point.

Lemma 12.4. Assume that $\left[\lambda_{1}, \lambda_{2}\right]$ is a closed subinterval of $\left(e_{1}, e_{2}\right)$, such that:

1) the itinerary of $c_{\lambda}$ is the same for each $\lambda \in\left[\lambda_{1}, \lambda_{2}\right]$ and not periodic,

2) $\omega_{\lambda}(c)$ does not contain parabolic periodic orbits, for $\lambda$ equal to either $\lambda_{1}$ or to $\lambda_{2}$.

Then the interval $\left[\lambda_{1}, \lambda_{2}\right]$ is not maximal obeying these properties.

In other words, if $\left[\lambda_{1}, \lambda_{2}\right]$ is a maximal non-trivial interval satisfying 1$)$, then: $\omega_{\lambda}(c)$ is non-minimal for each $\lambda \in\left[\lambda_{1}, \lambda_{2}\right]$, contains a periodic orbit, and in addition, $\omega_{\lambda}(c)$ contains a parabolic periodic orbit for both $\lambda$ equal to $\lambda_{1}$ and to $\lambda_{2}$.

Moreover, we have 
Lemma 12.5. Assume that $\left[\lambda_{1}, \lambda_{2}\right]$ is as in the previous lemma and $\Lambda^{\prime} c\left(\lambda_{1}, \lambda_{2}\right)$ is an open interwal such that $\omega_{\lambda}(r)$ conlatns no parabolic ryeles for all $\lambda \in \Lambda^{\prime}$. Then for every $\lambda \in \Lambda^{\prime}$, there is a first return map $G_{x}$ to a nice interval around a (depending on $\lambda$ ). such that all $G_{\lambda}$ are included in a family of box-mappings depending complexanalytically on $\lambda$ in a complex neighbourhood $\Omega$ of the open interval $\Lambda^{\prime}, \Omega \cap \mathbb{R}:=\Lambda^{\prime}$, and all maps $G_{\lambda}, \lambda \in \Omega$, are pairwise quasi-conformally rongugate.

Proof of Lemmas 12.4-10.5. If $\omega(c)$ is minimal, we nse Theorems A and B. and the further proof is a repetition of the proof of the infinitely renomalizable case of real analytic family of real analytic mimodal maps, see Sect. 7.2 of [Ko]. Note that condition 2) holds antomatically in this casse.

If $\omega(c)$ is not minimal, there is a sulsstantial difference caused by the fact that the box mapping we constructed in Theorm A las infinitely many branches, and in order to apply an idea of $[\mathrm{Ko}]$, we need to inchule this map in a complex analytic (on the parameter) family of such maps.

Let us assume that $\lambda_{2}=0$ and that $\omega_{0}(c)$ contains no parabolic (ycles. 'Then the map $g_{0}$ satisfies the conditions of 'Theorems $A$ ' and its ardendmm, and Theorem B'. Therefore, the first return map of $g_{0}$ to a nice interval $P(0)$ around $c, R_{0}: U P^{i}(0) \rightarrow$ $P(0)$, can be extended to a box mapping $F_{0}: U V^{i}(0) \rightarrow V(0)$, such that $V^{i}(0) \cap \mathbb{R}=$ $P^{i}(0), V(0) \cap \mathbb{R}=P(0)$. 'Zero' in the notations indicates that all this is constructed for the map $g_{0}$. Let us note that. 'Theorem A' shows that all intervals $P^{\prime}(0)$ are very deep inside $P(0)$, so that we can take $V(0)$ to be $D(P(0) ; \pi-\theta)$, where $\theta>0$ is small. We need the modification of $V(0)$ as made in the addendum to 'Theorem $A$ ', see Section 10. Namely, we replace (and fix, from now on) $V(0)$ by $\tilde{V}$, where $\tilde{V}$ is $D(P(0) ; \pi-\theta)$, except for two small neighbourhoods $Z_{1}, Z_{2}$ of the end points $a_{1}, a_{2}$ of the real trace $P(0)$ of $\tilde{V}$, so that $\partial \tilde{V}$ in $Z_{1}, Z_{2}$ is $g_{0}^{M}$-preimage of two leaves $l_{n_{1}}, l_{v_{2}}$ (invariant under an iterate of $g_{0}$ ) of the foliation defined in Section 10. As explained in the addendum to Theorem A', with the new range $V(0)$, the first return map to $V(0)$ is still well-defined and forms the initial box mal). Let us box mapping by $F_{0}: \cup V^{i}(0) \rightarrow V(0)$ (forgetting about the box mapping before this modification).

Let us consider the Yocco puzzle pieces of the map $F_{0}$ as defined in the Step 3 of the proof of Theoren $B$ (see the previous Section): the pieces of level $j \geq 0$ are by definition the components of $F_{0}^{-j}(V(0))$. In particular, we consider the critical pieces $V_{j}(0), j=1,2, \ldots$ of this partition (i.e., $V_{j}(0)$ is the component of $F^{-j}(V(0)$ ) containing $c$ ). Denote by $F_{j, 0}: \cup_{i} V_{j}^{i}(0) \rightarrow V_{j}(0)$ the first return mal, to $V_{j}(0)$ containing the points of $\omega(c) \cap V_{j}(0)$. (Our aim is to slow that, provided $j$ is large enough, one of the maps $F_{j, 0}$ can be included in a holomorphic fanily $F_{j, \lambda}$ (induced by $g_{\lambda}$ ), for $\lambda$ in a small (complex) neighloumhool of $\lambda=0$. 'The intea is the following. We include each individual component of $F_{j, 0}$ in a holomorphic: motion which agress with the $g_{\lambda^{-}}$ dynamics. On the other hand, provided $j$ is large, the dynamies of $F_{j, 0}$ is concentrated on a small neighbourhood of the real axis, and this persists for any individual motion. If now two motions intersect each other for some $\lambda$, then this intersection can be transferred to a fixed neightbourhood of the periodic point $a_{\lambda}$ of $y_{\lambda}$. Since the local dynamics of $g_{\lambda}$ repels a domain to a fixed distance away from the real axis, we obtain a contradiction. 
1. Let us include $\partial V(0)$ in a holomorphic notion $\phi_{\lambda}: \partial V(0) \rightarrow \partial V(\lambda),|\lambda|<\epsilon$, as follows.

Denote by $a_{\lambda}$ the periodic point of $g_{\lambda}$, which is a holomorphic extension of the periodic point $a=a_{0}$ of $g_{0}$, which was used to generate the real partition. Since $\omega_{0}(c)$ contains no parabolic points, $a_{0}$ is repelling. One can even assume that $a_{0}$ is a fixed point.

First of all, using that the linearization Böttcher coordinates around $a_{\lambda}$ depend holomorphically on $\lambda$, the leaves $l_{v_{1}}, l_{v_{2},}$ are naturally included in motions $l_{v_{1}}(\lambda), l_{v_{2}}(\lambda)$, so that $l_{\nu_{i}}(\lambda)$ are invariant nuder $g_{\lambda}^{-1}$ in a neightrourhood $Z$ of $a_{\lambda}$ which is fixed once and for all. This holomorphic motion is transferred by some branches of $g_{\lambda}^{-M}$ to neighbourhoods of $a_{i}(\lambda)$, where the branches are chosen so that $g_{0}^{-M}\left(a_{i}\right)=a_{i}(0)$ with $a_{i}(0)$ the end points of $P(0)$. In particular, the end points $Y_{i}(\lambda)$ of $g_{\lambda}^{-M}\left(l_{v_{i}}(\lambda)\right.$ ) (other than $\left.a_{i}(\lambda)\right)$ are holomorplic: functions of $\lambda$. The rest of $\partial V(0)$ between $Y_{i}(0), i=1,2$, is an are of the circle (and its complex conjugate). It can be included in a holomorphic motion as well within a family of ares of circles joining $Y_{i}(\lambda)$. We choose a complex neighbourhood $W_{0}$ of $\lambda=0$ so small that the end points of $l_{v_{i}}, i=1,2$, are some definite distance away from the real axis for all $\lambda \in W$. Then any point $x \in l_{v_{i}}, x \neq a_{\lambda}$ is repelled by iterates of $g_{\lambda}$ inside the neighbourhood $Z$ eventually to a definite distance $h>0$ away from the real axis, where $h$ is independent of $\lambda \in W_{0}$. Note that here we use that we work with the modified range $V$ with pieces of 'exterral rays' in the boundary, rather than the original range.

We shall constantly use the following obvious fact: Consider a univalent map $g_{0}^{n}$ : $A_{0} \rightarrow B_{0}$, where $A_{0}, B_{0}$ are domains with piecewise analytic boundaries and let $\phi_{\lambda}$ : $\partial B_{0} \rightarrow \partial B_{\lambda}$ be a holomorphic motion. Then, for any $\lambda$ in a complex neighbourhood of $\lambda=0$, the map $g_{\lambda}^{\prime \prime}: A_{\lambda} \rightarrow B_{\lambda}$ is also a well-defined mivalent map such that the domain $A_{\lambda}$ tends to $A_{0}$ as $\lambda \rightarrow 0$. In particular, Step 1 gives a holomorphic motion of each individual domain $V_{j}^{i}(0)$ defined by $g_{\lambda}^{-n_{i, j}} \circ \phi_{\lambda} \circ g_{0}^{n_{i, j}}: \partial V_{j}^{i}(0) \rightarrow \partial V_{j}^{i}(\lambda)$, where $g_{0}^{n_{i, j}}$ maps $V_{j}^{i}(0)$ onto $V(0)$. However, a priori, each such motion is defined in its own neighbourhood of $\lambda=0$.

2. Choose $j$ so large that the following holds: if $V_{j}^{i}(0)$ is any component of the map $F_{j}(0)$, then all iterates of $V_{j}^{i}(0)$ by $g_{0}$ until the range $V^{j}(0)$ of $F_{j}(0)$ lie within a $h / 10$-neighbourhood of the real axis (where $h>0$ has been defined in the step 1 ). To see this, use Fact 11.1, where we set $j=r(m)$ large enough. Indeed, then the moduli of the annuli $Q_{r(m)} \backslash V_{r(m)}$ are uniformly away from zero, and in particular $V_{r(m)}(0)$ shrinks to the critical point. By Fact 11.1 (b), with $x \in \mathbb{R}$, the statement follows.

3. With chosen $j$ as in the previous step, we forget about all other maps. So let us write $F_{0}, V^{i}(0)$ instead of $F_{j, 0}, V_{j}^{i}(0)$ (and so on). If (as for the minimal case) the number of components $V^{i}(0)$ is finite, then finitely many holomorphic motions already give us the desired extension. In our case, we also will obtain a holomorphic motion, because all, except finitoly many, components $V^{i}(0)$ of $F_{0}$ are concentrated around a real set $X(0)$, on which $g_{0}$ is expanding, and this situation persists for all $\lambda$ complex near 0 .

To make this precise, we consider the piece $P=P(0)$, and for this piece we consider the sets $X(0)=X$ and $\hat{X}(0)=\hat{X}$ refined in the end of Section 10, and the 
corresponding complex map

$$
G(0): \cup B(I) \rightarrow U D(I ; \pi-\beta):
$$

where $B(J)$ are finitely many pairwise disjoint topological discs all contained properly inside $\cup D(I ; \pi-\beta)$. Note that all $(C(0)$-itrentes of points of the sid. $\hat{X}(0)$ remain in the domain of definition of $G(0)$.

Now, let us construct the family $F_{X}$. For any component $V^{\prime}(0)$ of the map $F_{0}$ choose $m_{i} \geq 0$ minimal, such that $g_{0}^{m_{2}}\left(V^{i}(0)\right)$ is not property contiained in any domain $D(J)$. Then, since we have fixed $m_{0}$ in the construetion of the maj, $G$ in Section 10 ard since all iterates of all $V^{i}(0)$ until the lange $V(0)$ of $F_{0}$ ate rejther disjont or coincide, and are roughly dises, the number of different domains $g_{0}^{j}\left(V^{r}(0)\right), j-m_{i}, \ldots, N(i)$ (where $N(i)$ is such that $\left.g_{0}^{N i i}\left(V^{i}(0)\right)=V(0)\right)$ is bomuded (over all Vi $\left.(0)\right)$. Hence, all inferse branches of $g_{\lambda}$ leading from $V(\lambda)$ to these finitely many domains cau be well-defined in some small complex neighbourlood of $\lambda=0$. On the other land, all other branches (mapping from $g_{0}^{m i}\left(V^{i}(0)\right)$ to $\left.V^{i}(0)\right)$ persist for any other map $g_{\lambda}$, provided $\lambda$ is in a small complex neighbourlood of $\lambda=0$. The mason for this is that the map $G(0)$ is included in a complex analytic family $G(\lambda)$, where $\lambda$ is chose to $\lambda=0$, where we fix the range of $G(\lambda)$ for all such $\lambda$, becantse the mumber of componests of the donain of the map $G(0)$ is finite and their closures are patiowise disjoint. Therefore $G(\lambda)$ is uniformly expanding (where the expansion factor is bomeded by sone constant which does not depend on $\lambda$ and on the points). It follows, that lluere exists a smatl enomgh complex neighbourhood of $\lambda=0$, such that any $V^{\prime}(0), g_{0}^{\prime \prime \prime}\left(V^{\prime}(0)\right)=V(0)$, is inchuded in a holomorphic motion $V^{i}(\lambda)$. and $g_{\lambda}^{m_{i}}\left(V^{i}(\lambda)\right)=V(\lambda)$, and all $g_{\lambda}^{k}\left(V^{i}(\lambda)\right), k==0,1, \ldots, m_{i}$, remain in a $h / 5$-neighbourhood of the real axis.

4. It remains to show that these holomorphite motion of the individual components are pairwise disjoint with each other and with $\partial V(\lambda)$ for every $\lambda$ in a fixed neighb purhood of $\lambda=0$. Assume that the boundaries of $V^{i}(\lambda)$ and $V^{j}(\lambda)$ are not clisjoint for some $\lambda$ (close to 0$)$. In this case it is impossible that the sizes of both $V^{i}(\lambda), V j(\lambda)$ are smaller than some $r>0$ (independent on $\lambda$ ). This holds, becanse iterates of $\phi(\lambda)$ map such domains to disjoint domains. It is also intpossible that both $V^{i}(\lambda), V(\lambda)$ are larger than some constant $r^{\prime}>0$ when $\lambda$ is close to zero, because there are only finitely many large components. So, the only possible case for which we could have an intersection would be that $V^{i}(\lambda)$ is one of the $\tilde{N}$ big components (where $\hat{N}$ does not depend on $\lambda$ ), while $V^{j}(\lambda)$ is small (and the diameter of $V(\lambda)$ tends to zero as $\lambda \rightarrow 0)$. In particular, $g_{\lambda}^{K_{i}}\left(V^{i}(\lambda)\right)=V(\lambda)$, where $K_{\text {; }}$ are bomded from alowe by some universal $K$. To obtain a contradiction, olserve that in this situation, the iterates $g_{\lambda}^{k}\left(V^{j}(\lambda)\right), k=0,1, \ldots K$ lie in a small neighbourhood of the real interval, and that this neighbourhood even shrinks to the real axis as $\lambda \rightarrow 0$ (and the size of $V j(\lambda)$ tonds to zero as woll). Therefore, $g_{\lambda}^{K_{2}+M}\left(V^{j}(\lambda)\right)$ lies in a small neighbontoos of the fixed point $a_{\lambda}$ of $g_{\lambda}$, and, moreover intersects, say, the leaf $l_{n,}(\lambda)$ of the foliation (because we assumed that $V^{i}(\lambda), V^{j}(\lambda)$ did intersect and becallse $l_{h^{\prime}}(\lambda)$ is a piece of the boundanty of $\left.g_{\lambda}^{K_{i}+M}\left(V^{i}(\lambda)\right)\right)$. It follows that iterates of $g_{\lambda}$ repel a point of the domain $g_{\lambda}^{K_{i}+M}\left(V^{j}(\lambda)\right)$ (staying inside the neighboumood $Z$ of $a_{\lambda}$ ) to h-distance from the weal axis before returning to $V(\lambda)$. Ilowever, as shown in Step, 3 this does not happen, and so we proved by contradiction that domains remain disjoint. The same argmment also shows that 
domains are disjoint form the boundary of thes range, provided $\lambda$ is sufficiently close to 0 .

The rest of the proof repeats the consideration of Sect.7.2 of [Ko] (the infinitely renormalizable case), using Theorem $B$. If a is not recurrent, then we replace $F$ by the map $G$ constructed in Proposition 10.1 (so that it is not related to the piece $P$ anymore, but the Cantor repeller of $(r$ contains $\omega(c)$ is this case), and again repeat the construction of [ $\mathrm{Ki}(\mathrm{s}]$.

\section{Proof of Theorem B"}

In this section we want to prove Theorem B", which states that one has a quasisymmetric conjugacy on the entire dynamical interval when there are no parabolic or attracting periodic points.

Proof of Theorem $B^{\prime}$ : Let us for simplicity assume that the maps $g, \hat{g}$ are induced by covering maps of the circle $\mathbb{R} / \mathbb{Z}$. If there are gaps in the domain of these maps, then the proof is even simpler. The proof goes in four steps.

Step 1. Let ns first deal with the minimal case. Then in Theorem A we constructed a complex extension of the first return map to some nice interval $U_{k}$, but only considered the domains containing points in $\omega(c)$. Now consider the domains associated to extensions of ALL real clomains of the first return map to the nice intcrval $U_{k}$. Thus we get as in the proof of Theorem A a quasi-box mapping $P_{k}: \cup V_{k}^{i} \rightarrow V_{k}$. That the domains $\hat{V}_{k}^{i}$ intersecting $\omega(c)$ are containerl in $V_{k}$ follows from $|\omega(c)|=0$, see the proof of Theorem A. In order to see that the other domains are also contained in $V_{k}$, we need an improvement to the Schwar\% Lemma 2.3, which is due to E. de Faria and W. de Melo, see Lemma 2.4 in part II of [FM]. This improvenent states that there exists $\epsilon>0$ so that the spoiling factor in the Schwarz Lemma in the angles of the Poincaré domains when going from $g^{j+1}\left(V_{k}^{i}\right)$ to $g^{j}\left(V_{k}^{i}\right)$ is at most $\left(1+K\left|g^{j}\left(V_{k}^{i}\right)\right|^{1+\epsilon}\right)$. Since the orbit of $V_{k}^{i}$ up to its first entry in $V_{k}$ is disjoint and since the maximum size of the intervals in this orloit is small when $h$ is large, we get that $\prod_{j=1, \ldots, p}\left(1+K\left|g^{j}\left(V_{k}^{i}\right)\right|^{1+\epsilon}\right)$ can be assumed to be as close to 1 as we want (here $p$ is the return time of $V_{k}^{i}$ ). Hence $V_{k}^{i}$ is inside a disc with angle close to $\pi / 2$ (with some slits). Because the real trace of $V_{k}^{i}$ is well inside the real trace of $V_{k}$, we then obtain that $V_{k}^{i}$ is inside $V_{k}$. Thus wo obtain a quasi-box mapping with infinitely many domains, which can intersect and are contained (but not necessarily properly) inside $V_{k}$. The range $V_{k}$ is a disc with some slits: $V_{k} \cap \mathbb{R}=U_{k}$. Let us from now on fix $k$ and remove the $k$ from the notation. Consider a real one-paraneter family of ranges $V(t)$ by slightly changing the radius of the disc in such a way that $V(0)=V$ but so that the real trace is independent of $t$ Then we get a family of quasi-box mappings $P(t): \cup_{i} V^{i}(t) \rightarrow V(t)$ for $t \in \mathbb{R}$ sufficiently close to 0 . Now we want to obtain a box mapping from this. For this we proced as in Section 6. As in Step 1 of Section 6 , construct a smooth box mapping $g: \cup_{i} \hat{B}_{i} \rightarrow \hat{A}$, where $\hat{B}_{i} \cap \mathbb{R}$ are the (finitely many) domains of the definition of $g$. As above, consider the sequence of (all branches of the) first return maps to the central domains $\hat{g}_{j}: \cup_{i} \hat{B}_{i}^{j} \rightarrow \hat{A}^{j}$. In 
fact, we can choose $\hat{g}$ so that $A^{j} \cap \mathbb{R}=U_{j}$ for all $j$. To do this, take an invariant curve through one of the fixed points $p$ of $g$ (transversal to $\mathbb{R}$ ), then chense $A$ so that close to $p, \partial \hat{A}$ coincides with the invariant corve, and so that $g$ coincides with $g$ for all points in $A$ close to the real line. Then extend $y$ smoothly as in Section 6 . Next, as in Step 2 of Section 6, intersect the smooth box mapping $i_{j}$ with the (prasi-box mapping $P(t)$ (where we assume that $j$ is chesen so that two ranges $A^{\prime}$ and $V$ correspond to the same level). By the 'Theorem of Sard (which states that the set of eritical values of a smooth mapping $h: \mathbb{R} \rightarrow \mathbb{R}$ has lobesgne measure zero). it follows that, we can find $t$ close to zero, so that the ranges of $P^{\prime}(t)$ and $\hat{g}_{j}$ (which are buth precewise monoth curves) are transversal. In liat, the range $l^{\prime}(t)$ hand two slits but since we made sure that $\hat{A}^{j} \cap \mathbb{R}=U_{j}$, we get that the component of $A^{j} \cap V(t)$ containing $\mathrm{s}$ is a topological circle consisting of a finite number of smootly ares and without cuspes (this follows from the transversality). So we obtain a box mapping $P: U_{i} \Omega^{\prime} \rightarrow \Omega$ so that $O \Omega$ is a quasicircle. $P$ is an iterate of $g$ ou each of the domains, hile domains are all disjoint, and properly contained in $\Omega$. We do not elaim that the modulus of the anmuli $\Omega \backslash \Omega \Omega^{i}$ can be bounded away from zero uniformly in $i$. However, by passing to the first return map to the central domain this modulus condition will be satisfied: the "safe-space' condition 4 from Theorem A' in Section 10 holds. If we are in the nom-minimal ase, then we can also extend the box mapping from 'Theorem $\mathrm{A}^{\prime}$ to a box mapping $P: \cup_{i} \Omega^{i} \rightarrow \Omega$ such that the closure of $\cup_{i} \Omega^{i} \cap \mathbb{R}$ again contains $\Omega \cap \mathbb{R}$. T'o prove this, we do not need the smooth polynomial-like mappings, but only that the real domains $\Omega^{i} \cap \mathbb{R}$ are deep inside $\Omega \cap \mathbb{R}$ (see the proof of 'Theorem A'). Both in the minimal and non-minimal case let $A^{+} . A^{-}$be the set of points in the npperhalf (lowerhalf) plane which are inside $\Omega$ but ontside all the domains $\Omega^{\prime}$ and let $A=A^{+} \cup A^{-}$.

Step 2: Now observe that the bommlarios of $A^{+}$and of $A^{-}$are culasicincle. The proof of this is identical to that in Step 1 of section 11 becanse the houndary of $\Omega$ is a quasicircle, and because each of the domains $\Omega^{i}$ is mapped with uniformly bounded distortion to $\Omega$.

Step) 3: In Theorems $B$ and $B^{\prime} w($ did ohtain a quasiconformal map $\phi: \Omega \rightarrow \tilde{\Omega}$ conjugating $P: \cup_{i \in J} \Omega^{i} \rightarrow \Omega$ aud $\tilde{P}: \cup_{i}, \Omega^{i} \rightarrow \bar{\Omega}$ where the iudex set $J$ corresponds to the domains interserding $\omega(c)$. Take any glusiconformal map $\psi: \Omega-i \Omega$ extending the restriction of $\phi$ to $\partial \Omega$. Let $K$ be its quasieonformal dilatation. By pulling back once, we obtain also $K$-quasiconformal maps $d_{i} ; \Omega^{i} \rightarrow \Omega^{i}$ for each $i \notin J$. As in Step 2 of the proof of 'Theorem B' we can find a quasiconformal conjugary $I I: A \rightarrow \tilde{A}$ which agrees with $\phi$ on $\partial \Omega^{i}$ for $i \in J$ and with $\psi_{i}$ on $\partial \Omega^{i}$ for $i \notin J$. Now extend $H: A \rightarrow \tilde{A}$ to $H: \Omega \rightarrow \tilde{\Omega}$ as follows. On $\cup_{i \in J} \Omega^{i}$ let $/ / \mathrm{l}$ or expal to the conjugacy $\phi$ and on $\Omega^{i}$ with $i \notin J$ let $H$ be ecpual to $\psi_{i}$. As in Fact 11.2, we obtain that $H: \Omega \rightarrow \Omega$ is quasiconfontual. $H$ is a conjugacy on the domains $\Omega^{i}$ intersed ting $\omega(c)$. If $g$ is minimal, then the domains where $H$ is a conjugacy cover $\omega(c) \cap \Omega$. So by taking subsecpuent pullbacks (as in the pron of Fact 11.2 but since we already have a quasiconformal ronjugacy on $\omega(c)$ the dilatation now will not depend on $n$ ), we obtain a secuence of (fuasiconformal homeomorphisms $H_{n}: \Omega \rightarrow \bar{\Omega}$ with the same (1uasiconformal dilatation and converging to a renjugacy $\Phi$ between $P: \cup \Omega^{i} \rightarrow \Omega$ and $\tilde{P}: \dot{\Omega}^{i} \rightarrow \Phi$. If g is mon-minimal, then the construction of a conjugacy is already done in Steres 3 and 4 of the proof of Theorem B' (simply also inchude the domains which do not. intersed w(c); the proof is identival). 
Step 4: There exists a minimal $N$ so that the $y^{N}$ maps $\Omega \cap \mathbb{R}$ injectively onto the whole dynamical interval. By taking, if meessary, puzble pieces of deeper level in $\Omega$ which intersect the real segment $\Omega \cap \mathbb{R}$, we can makn sure that the image under $g^{N}$ of all these puzzle pieces are disjoint. Their nuim is the necklace neighbourhood mentioned in the statement of the theorem. (Of comme the conjugacy can be naturally defined (extending the previous conjugacy) quasiconformally to this nenghbourhood by using $\tilde{g}^{N} \circ \Phi \circ g^{-N}$. Using the saure composition, it also follows that any point of the real line has a nejghbouthood on which the conjugacy extends quasiconformally. By compactuess of the lynamical interval, it follows that his cuas conformal map is defined on a neighbourlood of the dynamieal interval. Tlierefore. the conjugacy on the real line is a quasisynmetry.

\section{Appendix}

As before, let $J$ be a real interval and $D_{*}(J)$ the dise with diameter $J$. Consider the inverse by $I^{\prime}(z)=z^{f}$ of $D_{*}\left(\left[-1, K_{0}^{\prime}\right]\right) \backslash \mathbb{R}^{-}$with $K_{0}>1$. The next lemma states that the component of this inverse which contains $\left[0 . K_{0}\right]$ is contained in $D_{*}\left(\left[-1, K_{0}\right]\right)$.

Lemma 14.1 Let $P_{r^{\prime}}(z)=z^{\prime}$ where $\ell \geq 2, \cdots 1<0<1<K_{0}$ and

$$
W=\{z \in \mathbb{C} ; \arg (z) \notin[\pi(1-1 / \ell), \pi(1+1 / \ell)]\} .
$$

Then

In particular, some subset of

$$
P_{i}^{\cdots-1}\left(D_{*}\left(\left[-1 . K_{0}^{-i}\right]\right)\right) \cap W C_{*}\left(\left[-1, K_{0}^{*}\right]\right)
$$

$$
D_{*}\left(\left[-1, K_{0}\right]\right) \cap\{z \in \mathbb{C} ; \arg (z) \in[-\pi / \ell, \pi / \ell]\}
$$

is mapped diffeomorphically onto

$$
D_{*}\left(\left[-1, K_{0}^{*}\right]\right) \backslash \mathbb{R}^{-\cdot}
$$

Proof: Rescale $\left[-1, K_{0}\right]$ to $[-\Delta, 1]$ and so consider the disc $D$ with diameter $[-\Delta, 1]$, where $\Delta<1$, and define $P(z)=z^{2}$. Let us show that $D$ is contained in $P(D)$ (and that the only intersection point is $z=1$ ). Let ns parameterize the boundary by $\alpha \mapsto r(\alpha) \cdot e^{i \alpha}$. We want to show that $r^{2}(\alpha)>r(2 \alpha)$ except that equality holds if $r=1$ (i.e. $\alpha=0$ ).

Using the cosine rule we get

$$
\begin{gathered}
r^{2}(\alpha)+\frac{(1-\Delta)^{2}}{4}-r(\alpha)(1-\Delta) r(\alpha)(\alpha)=\frac{(1+\Delta)^{2}}{4}, \text { i.e. } \\
r^{2}(\alpha)-r(\alpha)(1-\Delta) \cos (\alpha)-\Delta=0 .
\end{gathered}
$$

Hence,

$$
\cos (\alpha)=\frac{r^{2}(\alpha)-\Delta}{r(\alpha) \cdot(1-\Delta)} \text { and }
$$




$$
\cos (2 \alpha)=2\left(\frac{r^{2}(\alpha)-\Delta}{r(\alpha) \cdot(1-\Delta)}\right)^{2}-1
$$

Similarly, the point $r(\alpha) \cdot r^{i 2 \alpha}$ satisfies

$$
r^{2}(2 \gamma)-r(2 \alpha)(1-\Delta) \cos (2 \alpha)-\Delta=0
$$

Let us write $A=r^{2}(\alpha)$ and $B=r(2 \alpha)$ and substitute the expression for $\cos (2 \alpha)$ in the last expression. Then we get.

$$
B^{2}-B(1-\Delta)\left[2 \frac{(A-\Delta)^{2}}{A(1-\Delta)^{2}}-1\right]-\Delta=0
$$

This means

$$
\frac{(1+B)(B-\Delta)}{B(1-\Delta)}=\frac{B^{2}-\Delta}{B(1-\Delta)}+1=\frac{2(A-\Delta)^{2}}{A(1-\Delta)^{2}}
$$

If $A=B:=C$ then this gives the following linear equation in $C^{\prime}$

$$
(1+C)=2 \frac{(C-\Delta)}{(1-\Delta)}
$$

which is only satisfied if $C=1$. This means that the only intersection of $D$ with $P(D)$ is at $z=1$. To show that $\partial P(D)$ is ontside $\partial D$ (except at $z=-1$ ). let us analyze the situation close to $z=1$. So regard in (14.2) $B$ as a function of $A$ and rlifferentiate $B$ w.r.t. $A$ at $A=1$ (and so also take $B=1$ ). 'This gives

$$
2 B^{\prime} B-B^{\prime}(1-\Delta)\left[2 \frac{(A-\Delta)^{2}}{A(1-\Delta)^{2}}-1\right]-B \frac{2}{(1-\Delta)}\left[1-\Delta^{2} / A^{2}\right]=0,
$$

i.e., since $A=B=1$,

$$
2 B^{\prime}-B^{\prime}(1-\Delta)-2 \frac{\left(1-\Delta^{2}\right)}{(1-\Delta)}=0
$$

which gives $B^{\prime}=2$ and so $B(A)<A$ for $A<1$.

This, and Lemma 15.2 from [LS1] inply the proof of this lemma.

\section{References}

$[\mathrm{BH}] \quad$ B. Bramner and J.H. Hubland, The iteration of cubie polynomials. Part II. Acta Matl. 169 (1992), 229-325.

[DH] A. Donady and J.II. Hubbard, On the dymamics of polynomial-like mappings. Anu.Sc.E.N.S. 4 serie 18, 287-343 (1985).

[FM] E. de Faria and W. de Melo, Rigidity of critical chrele mapings, part I and part $I$, preprints $1997 / 16$ and 1997/17 Stony Brook. 
[GS1] J. Graczyk and G. Swiatek, Polynomial-like properties for reai quadratic polynomials, preprint 1995, published in Topology Proceeding 21 (1996).

[GS2] J. Graczyk aud G. Swiatek, Generic hyperbolicity in the logistic family, Annals of Math. 146, 1-52 (1997).

[He1] M. Herman, Sur la conjugaison differentiable des diffcomorptismes du cercle a des rotations, Publ.Math. I.H.E.S. 49, 5-234 (1979).

[He2] M. Herman, Unpublished manuscript.

[HK] J. Heinonen and P. Koskela, Definitions of quasiconformality. Inv. Math. 120, $61-79(1995)$.

[Ko] O. Kozlovski, Structural stability in one-dimensional dynamics. Thesis, Amsterdam University, Jamuary 1998.

[Le] G. Levin, Bounds for maps of an interwal with one reflecting critical point. I. Fundamenta Math. 157, 287-298 (1998).

[Le1] G. Levin, On successiue bifurcations of one-parametric families of maps, Russian Mattl. Surveys 37, No. 2, 211-212 (1982).

[LS1] G. Levin and S. van Strien, Local ronnectivity of the Julia set of real polynomials, Preprint December 1994 and Stony Brook preprint 1995/5. Annals of Math. 147, 471-541 (1998).

[LS2] G. Levin and S. van Strien, Total disconnectedness of the Julia set of real polynomials, Preprint IHES, 1996/68 (1996). To appear in Asterisque.

[LY] M. Lyubich and M. Yampolski, Complex bounds for real maps. Preprint 03495 MSRI.

[Ly] M. Lyubich, Dynamics of quadratic polynomials I-II, Acta Matl. 178, 185-297 (1997).

[LM] M. Lyubich and J. Milnor, The unimodal Fibonacci map, Journal of the A.M.S. 6, 425-457 (1993).

[LeVi] O. Lehto and K.I. Virtanen, Quasiconformal mappings in the plane, SpringerVerlag, 1973.

[Ma] M. Martens, Interval dynamics, Thesis, Delft Technical University, (1990).

[McM1] C. McMullen, Complex dynamics and renormalization, Princeton Univ. Press, Ann. Math. Studies 135 (1994).

[McM2] C. McMullen, Renormalization and 3-Manifolds which Fiber of the circle, Princeton Univ. Press, Ann. Math. Studies 142 (1996). 
[Mil] J. Milnor, Local connectimity of Julia sets; expository lectures, Stony J3rook Preprint Series, 1992/11.

[MS] W. de Melo and S. van Strien, One-dimensionul dynamies, Ergelnisse Series 25, Springer-Verlag, 1993.

[MP] W. de Melo and A. A. Pinto, Rigidity of $\mathrm{C}^{2}$ infinitely mormalizable unimodal maps, Stony Brook Prepriut Series, 1999/6.

[Sa] D. Sands, Unpublished, 1996.

[Sh] M. Shishikura, Manuscript, 1998.

[Str] S. van Strien, Hyperbolicity and imvariant mosusures for general $C^{2}$ intervals maps satisfying the Misturewicz condition, Commm. Math. Phys. 128, 437496 (1990).

[Su] D. Sullivan, Bounds, quadratic differentials, and rmormalization conjectures, 1990. Published in AMS Centennial Publications. 2: Mathematics into Twenty-first Century.

[Stre] J. Strebel, On the matimal dilatation of quasiconfonmal mappings, Prockedings of AMS, 6 (1955), 903-909.

[Ya] M. Yampolski, Complex bounds for critical circle maps, Preprint Stony Buook $1995 / 2$.

[Yo] J.-C. Yoccoz, Uupublished. 